\title{
Redox-Controlled Reactivity at Boron: Parallels to Frustrated Lewis/Radical Pair Chemistry
}

\author{
Anthony Wong, ${ }^{\dagger, \S}$ Jiaxiang Chu, ${ }^{\dagger, \ddagger, \S}$ Guang Wu, ${ }^{\dagger}$ Joshua Telser, $\|$ Roman Dobrovetsky, ${ }^{\circledR}$ and \\ Gabriel Ménard*,† \\ ${ }^{\dagger}$ Department of Chemistry and Biochemistry, University of California, Santa Barbara, California 93106, United \\ States \\ ${ }^{\ddagger}$ School of Chemical Science, University of Chinese Academy of Sciences, Huaibei hen, Huairou District, Beijing \\ 101408, China \\ " Department of Biological, Chemical, and Physical Sciences, Roosevelt University, Chicago, Illinois 60605, United \\ States \\ §School of Chemistry, Raymond and Beverly Sackler Faculty of Exact Sciences, Tel Aviv University, Tel Aviv 69978, \\ Israel
}

\section{Table of Contents}

1. Supplemental Figures for NMR Spectroscopy......................................................................... 2

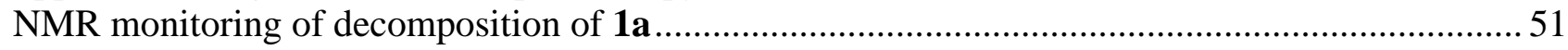

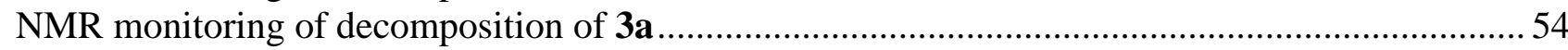

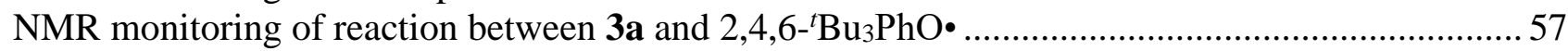

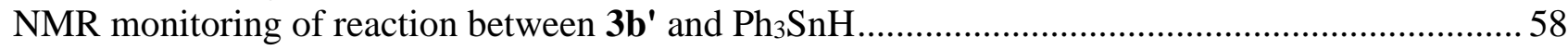

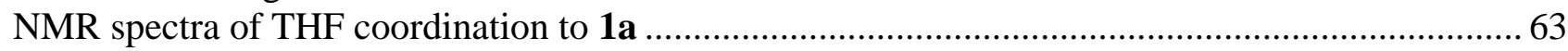

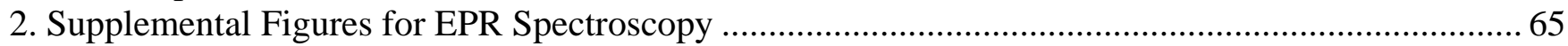

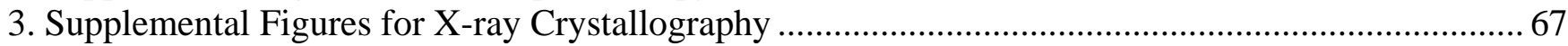

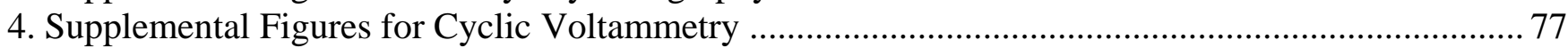

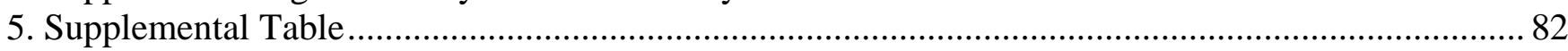

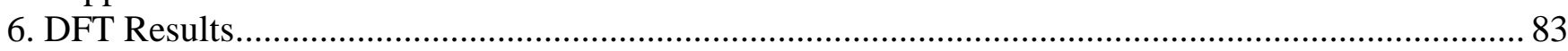

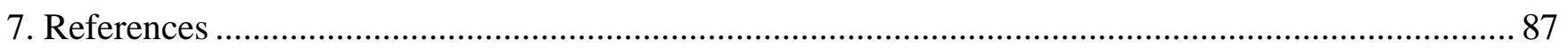


1. Supplemental Figures for NMR Spectroscopy

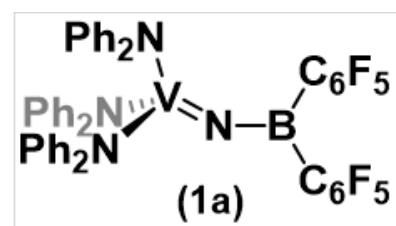

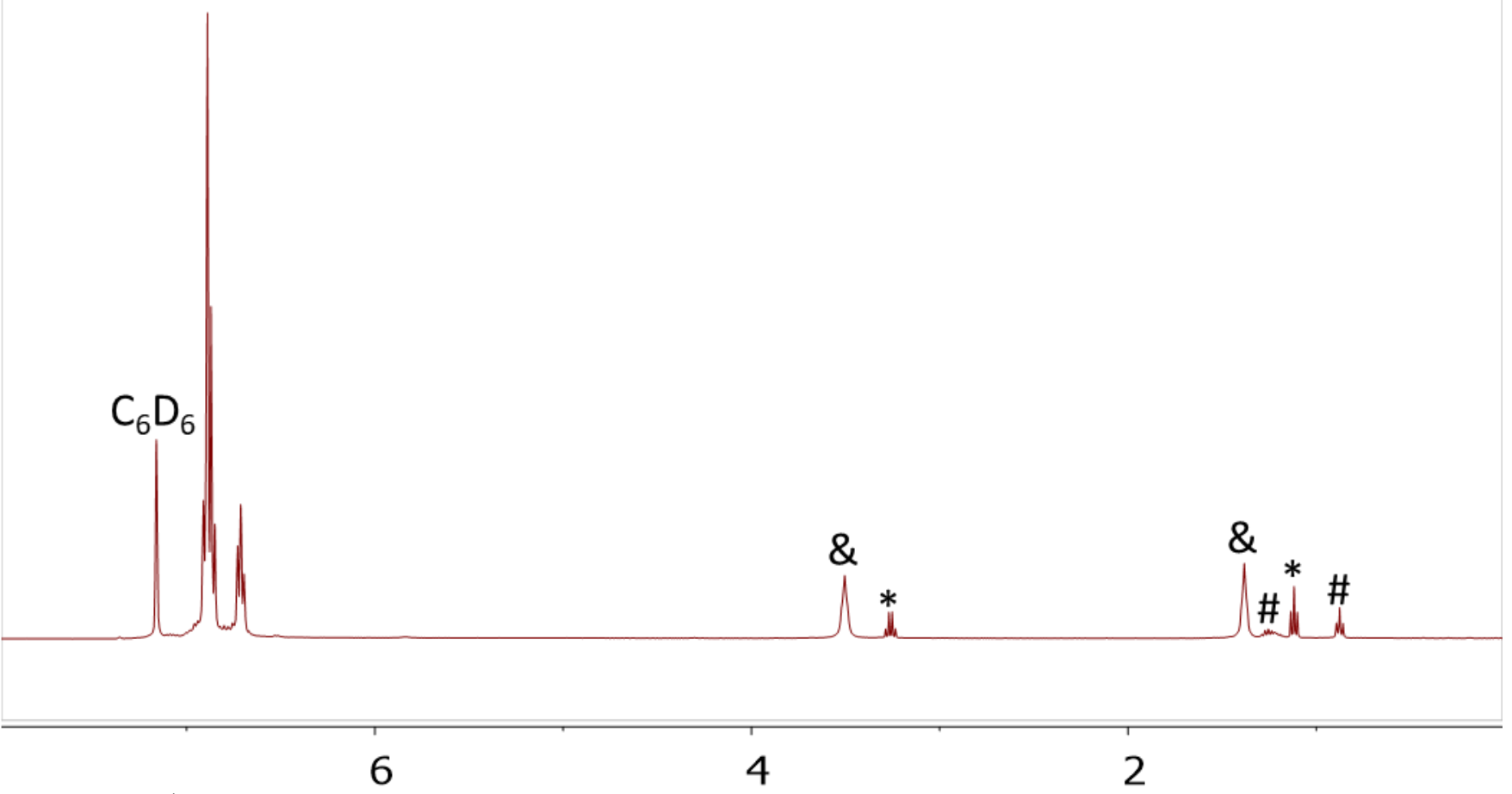

Figure S1. ${ }^{1} \mathrm{H}$ NMR spectrum of $\mathbf{1 a}\left(400 \mathrm{MHz}, \mathrm{C}_{6} \mathrm{D}_{6}, 25^{\circ} \mathrm{C}\right) .(\#=$ pentane, $*=$ ether, $\&=\mathrm{THF})$ 


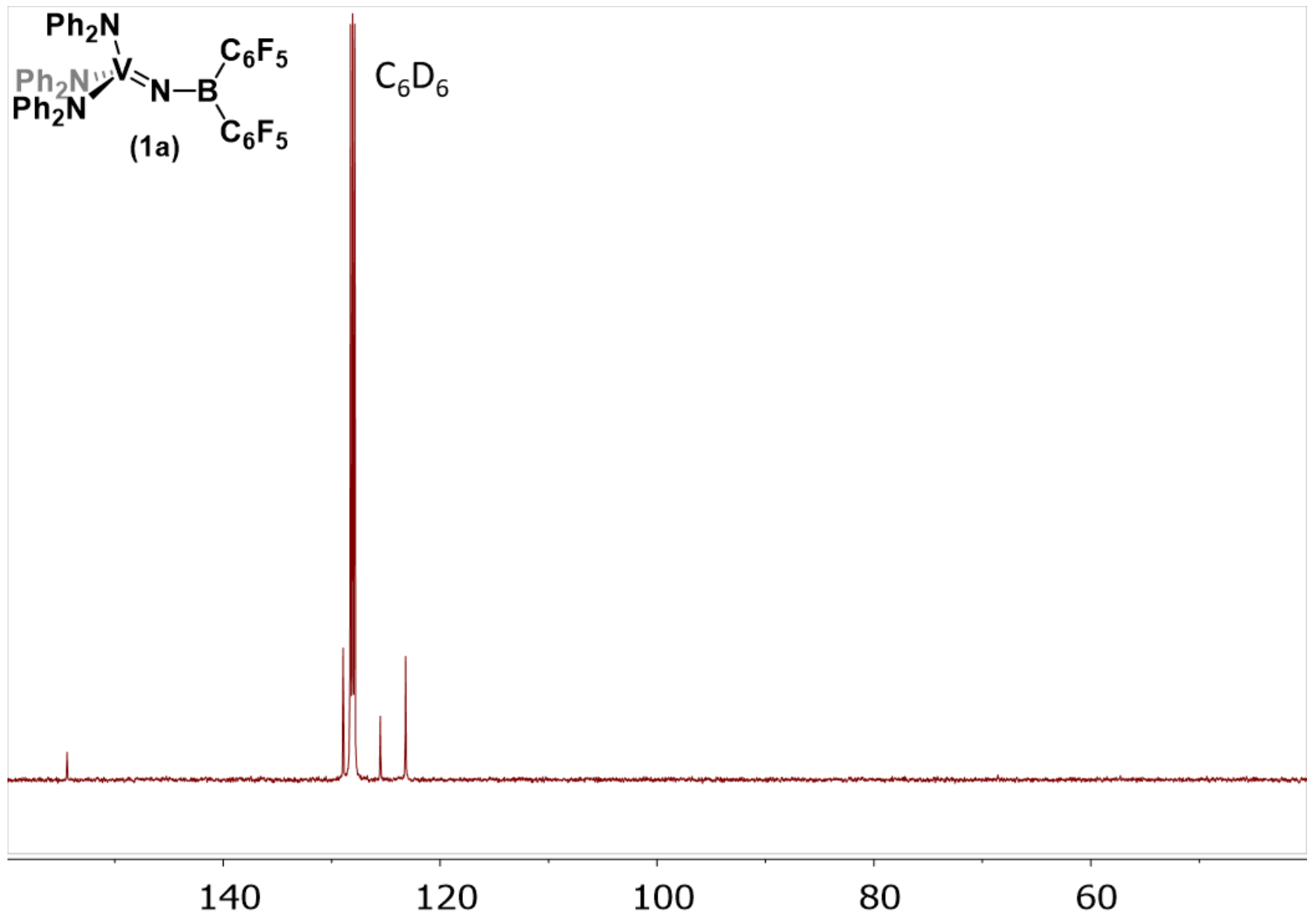

Figure S2. ${ }^{13} \mathrm{C}\left\{{ }^{1} \mathrm{H}\right\}$ NMR spectrum of $1 \mathbf{a}\left(100 \mathrm{MHz}, \mathrm{C}_{6} \mathrm{D}_{6}, 25{ }^{\circ} \mathrm{C}\right)$. 

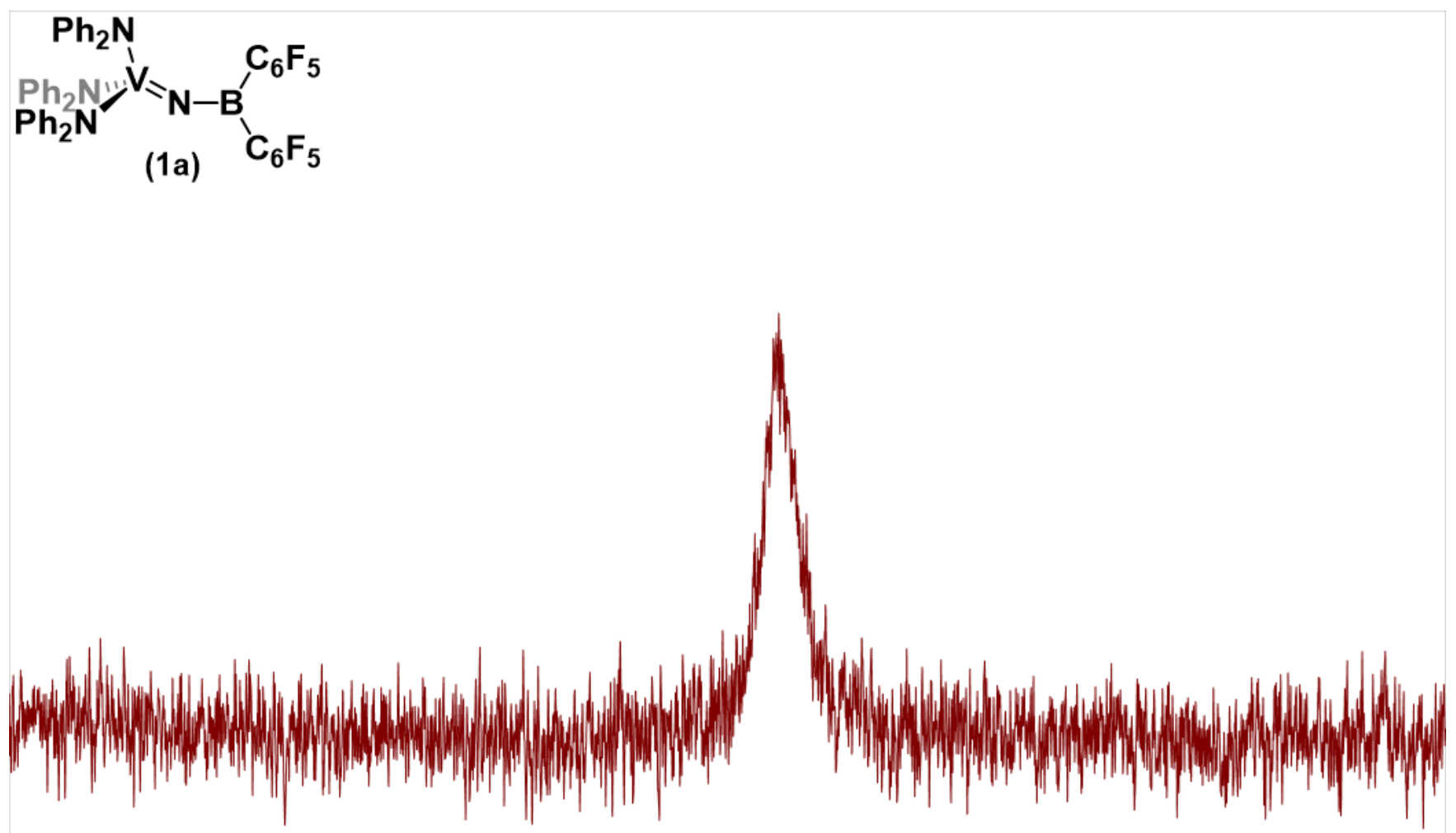

150

100

Figure S3. ${ }^{51} \mathrm{~V}$ NMR spectrum of $\mathbf{1 a}\left(105 \mathrm{MHz}, \mathrm{C}_{6} \mathrm{D}_{6}, 25^{\circ} \mathrm{C}\right)$. 


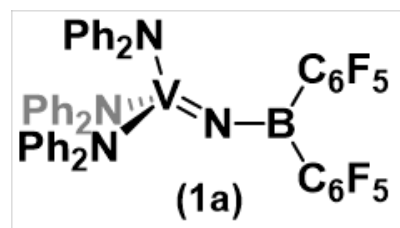

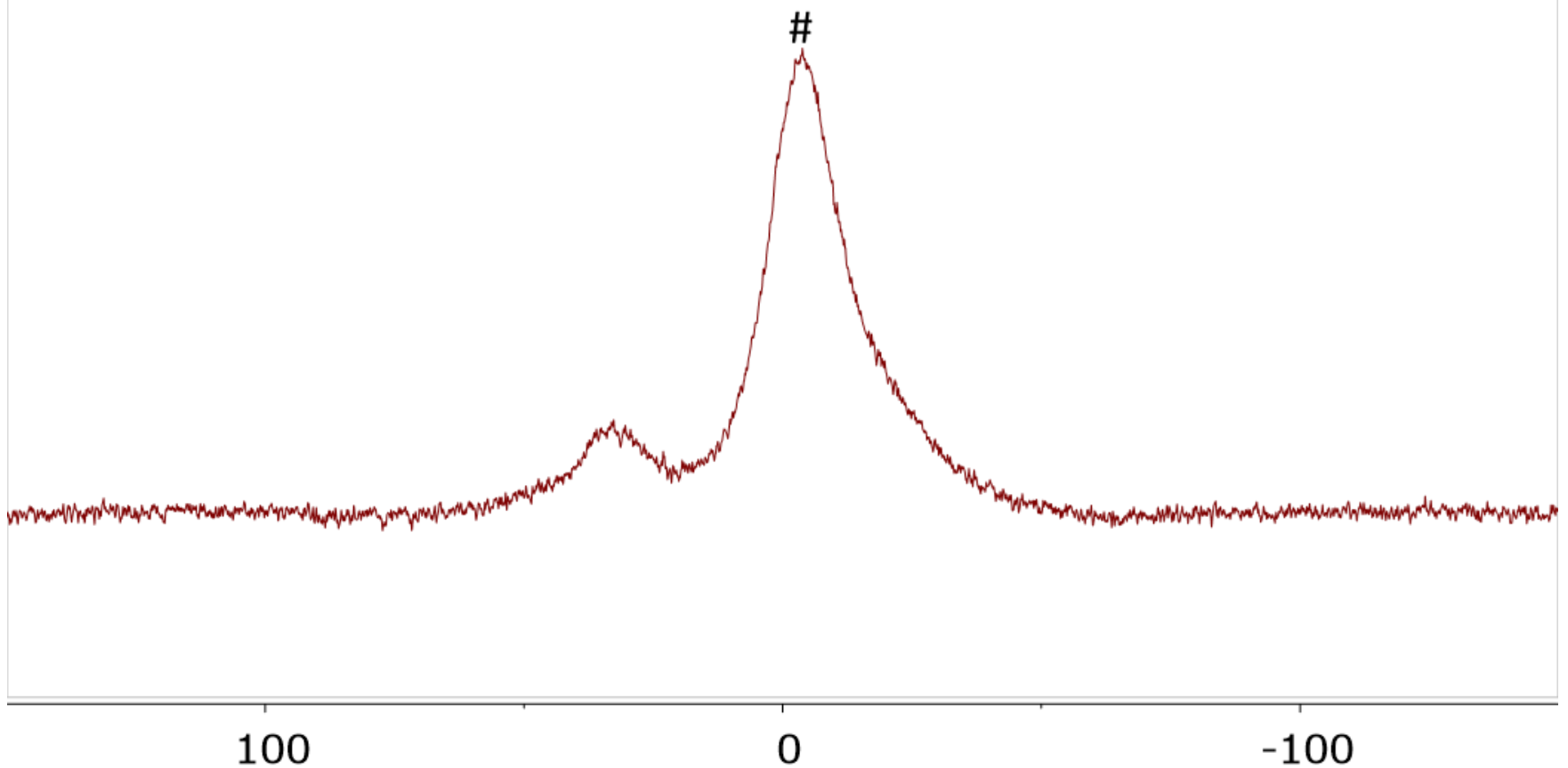

Figure S4. ${ }^{11} \mathrm{~B}\left\{{ }^{1} \mathrm{H}\right\}$ NMR spectrum of $1 \mathrm{a}\left(128 \mathrm{MHz}, \mathrm{C}_{6} \mathrm{D}_{6}, 25{ }^{\circ} \mathrm{C}\right)$. (\# = borosilicate resonance from NMR tube) 

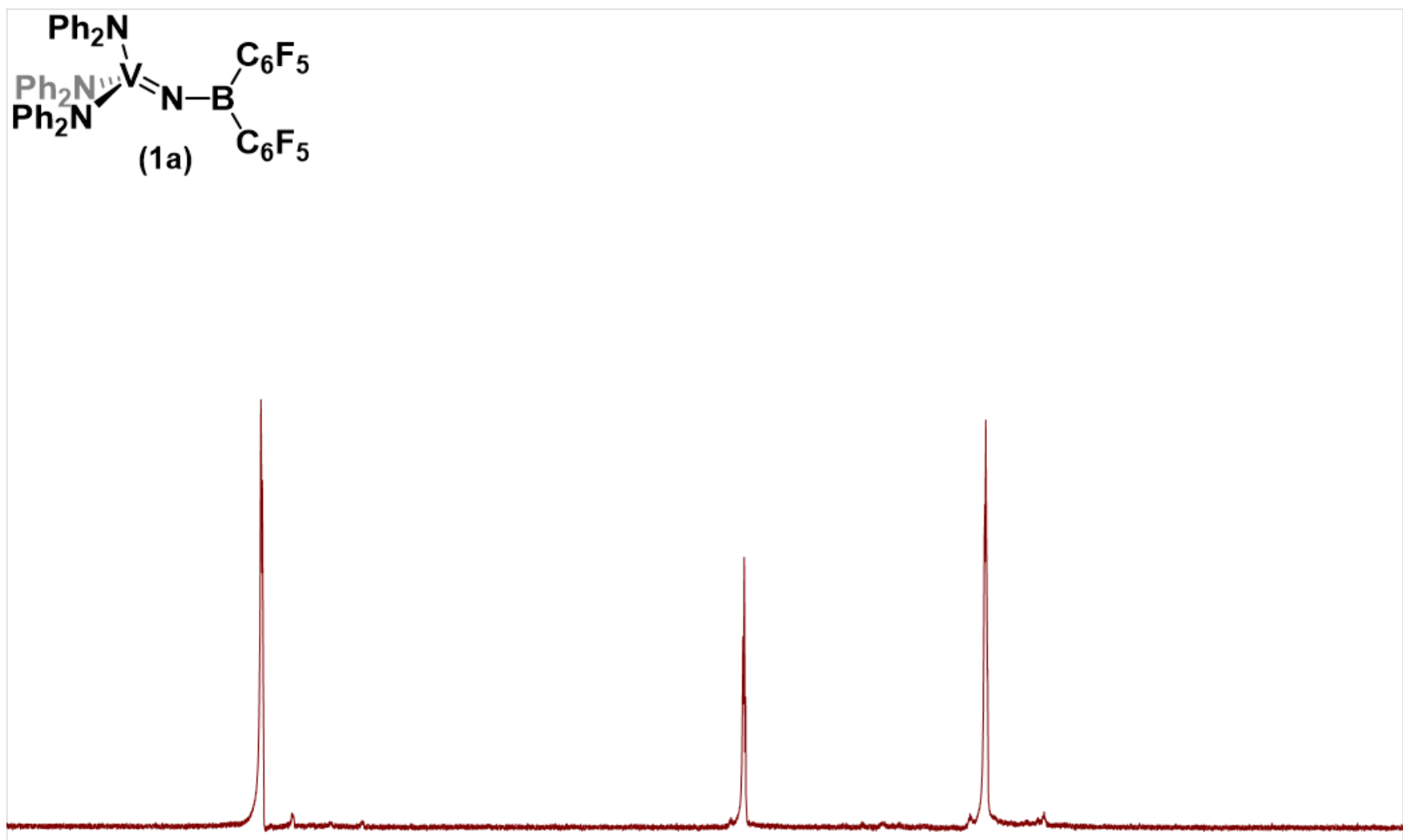

$$
-130
$$

$-140$

$-150$

$-160$

$-170$

Figure S5. ${ }^{19} \mathrm{~F}\left\{{ }^{1} \mathrm{H}\right\}$ NMR spectrum of $\mathbf{1 a}\left(376 \mathrm{MHz}, \mathrm{C}_{6} \mathrm{D}_{6}, 25^{\circ} \mathrm{C}\right)$. 


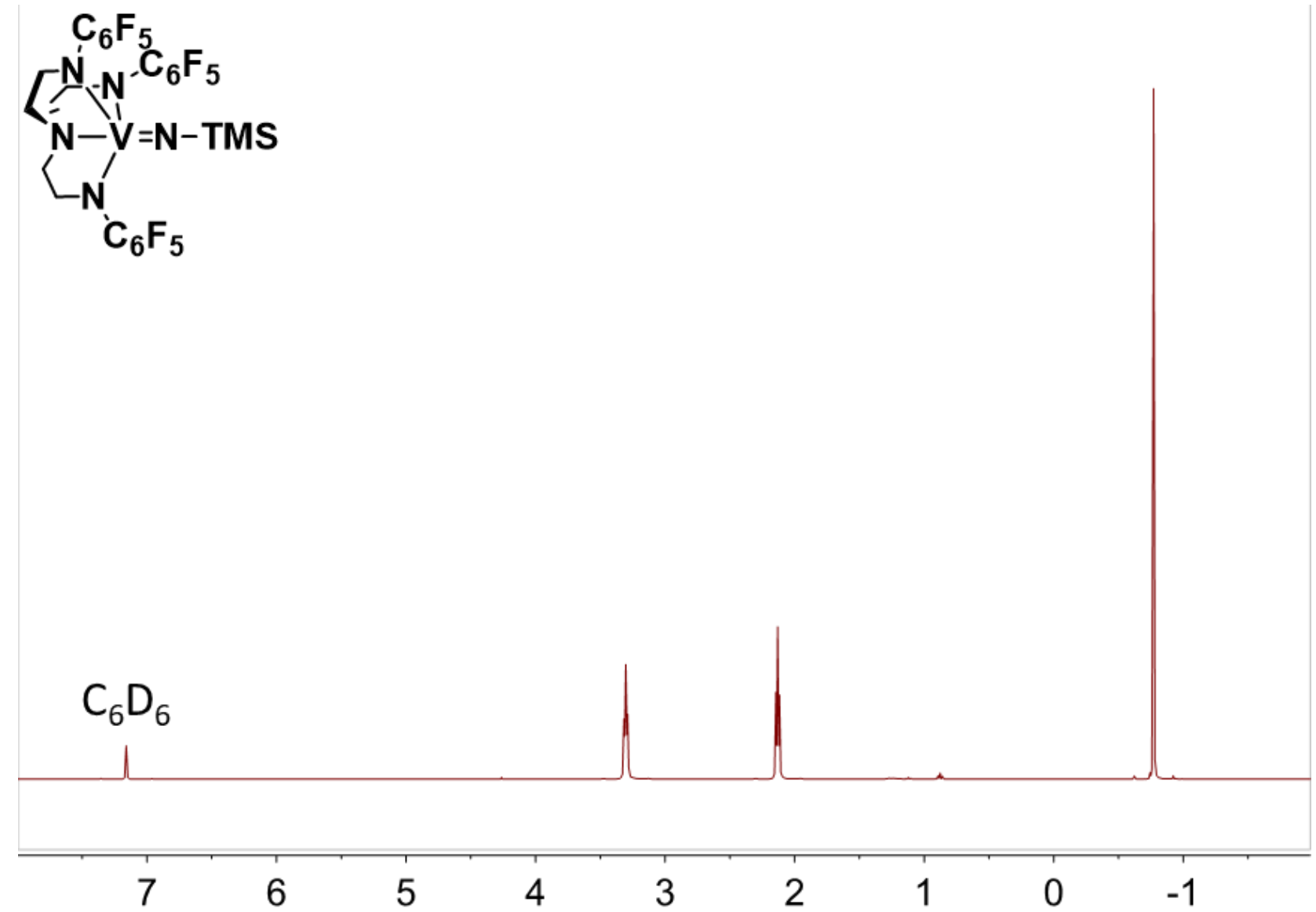

Figure S6. ${ }^{1} \mathrm{H}$ NMR spectrum of (tren)VNTMS (400 MHz, $\left.\mathrm{C}_{6} \mathrm{D}_{6}, 2{ }^{\circ} \mathrm{C}\right)$. 


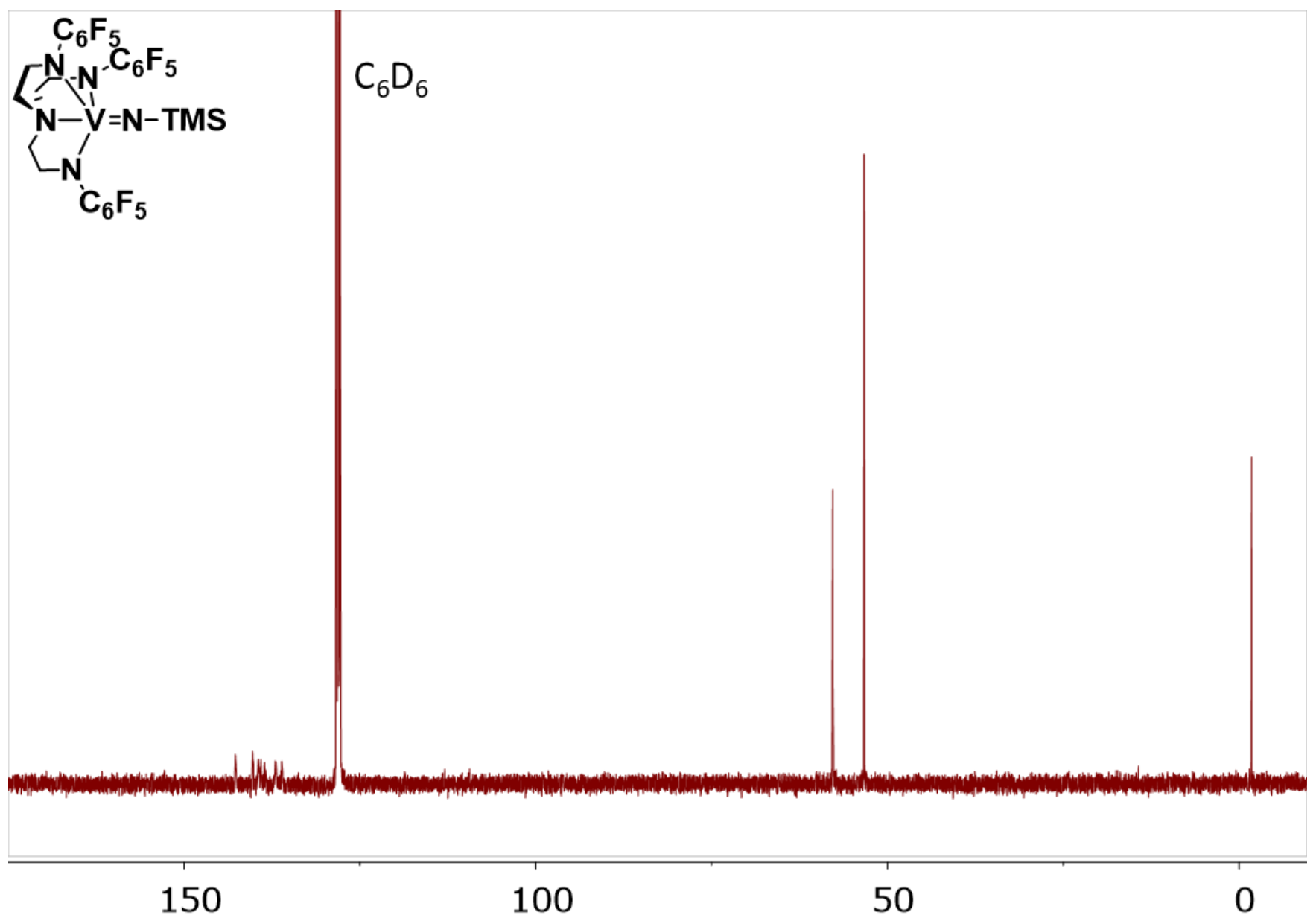

Figure S7. ${ }^{13} \mathrm{C}\left\{{ }^{1} \mathrm{H}\right\}$ NMR spectrum of (tren)VNTMS $\left(100 \mathrm{MHz}, \mathrm{C}_{6} \mathrm{D}_{6}, 25{ }^{\circ} \mathrm{C}\right)$. 

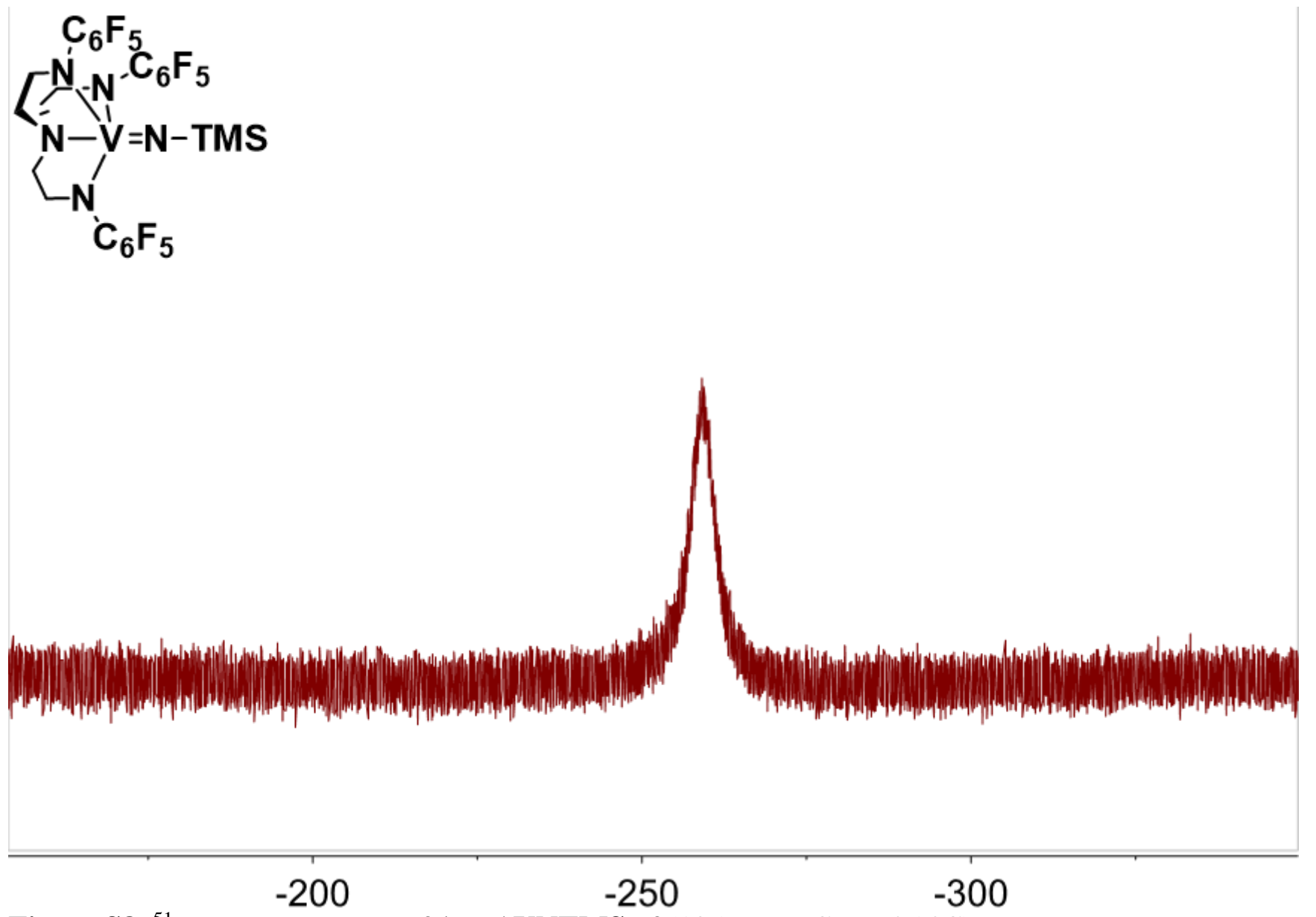

Figure S8. ${ }^{51} \mathrm{~V}$ NMR spectrum of (tren)VNTMS of (105 MHz, 66 D6, $\left.25{ }^{\circ} \mathrm{C}\right)$. 


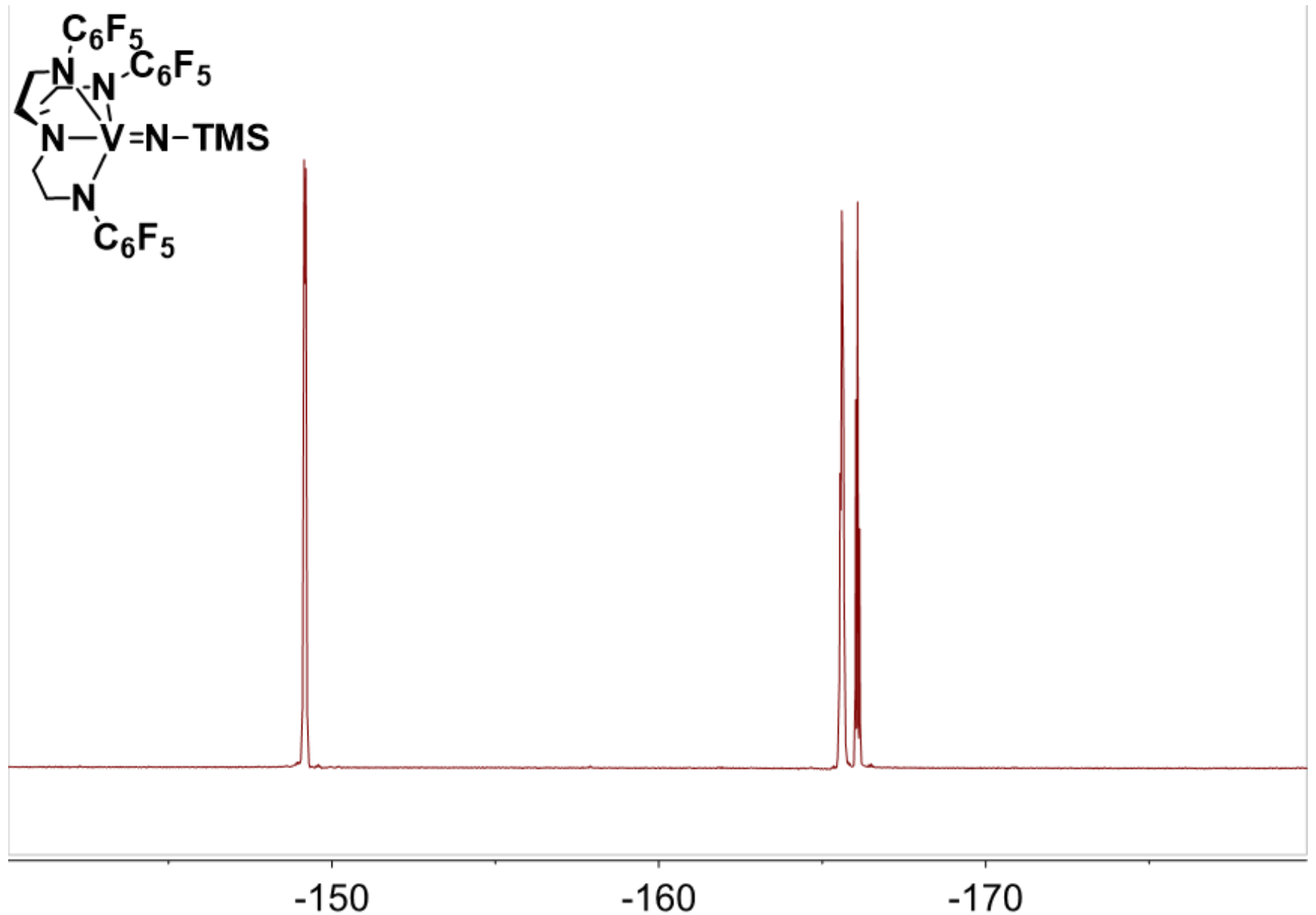

Figure S9. ${ }^{19} \mathrm{~F}\left\{{ }^{1} \mathrm{H}\right\}$ NMR spectrum of (tren)VNTMS $\left(376 \mathrm{MHz}, \mathrm{C}_{6} \mathrm{D}_{6}, 25{ }^{\circ} \mathrm{C}\right)$. 


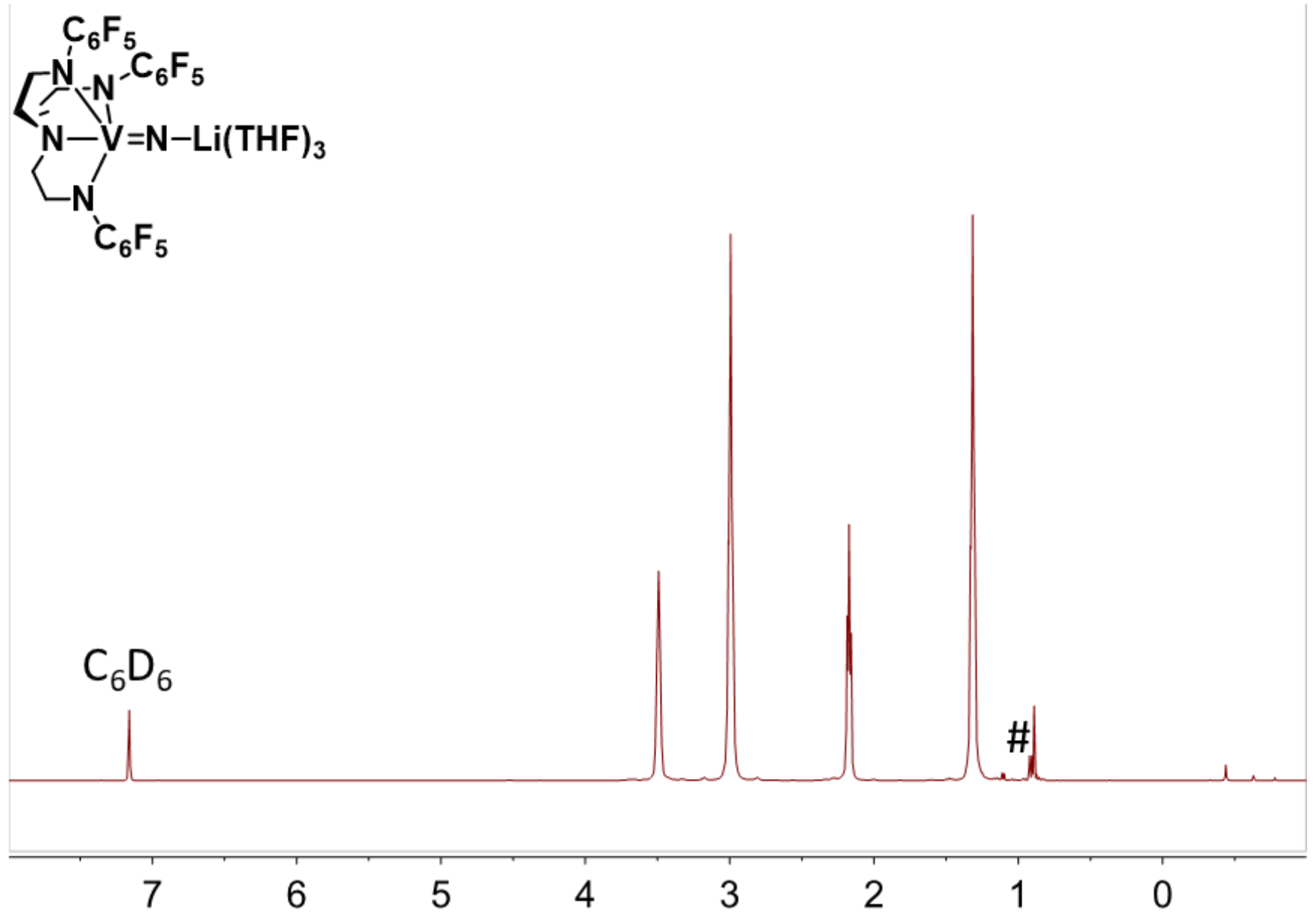

Figure S10. ${ }^{1} \mathrm{H}$ NMR spectrum of (tren)VNLi $\left(400 \mathrm{MHz}, \mathrm{C}_{6} \mathrm{D}_{6}, 25{ }^{\circ} \mathrm{C}\right) .(\#=$ pentane) 


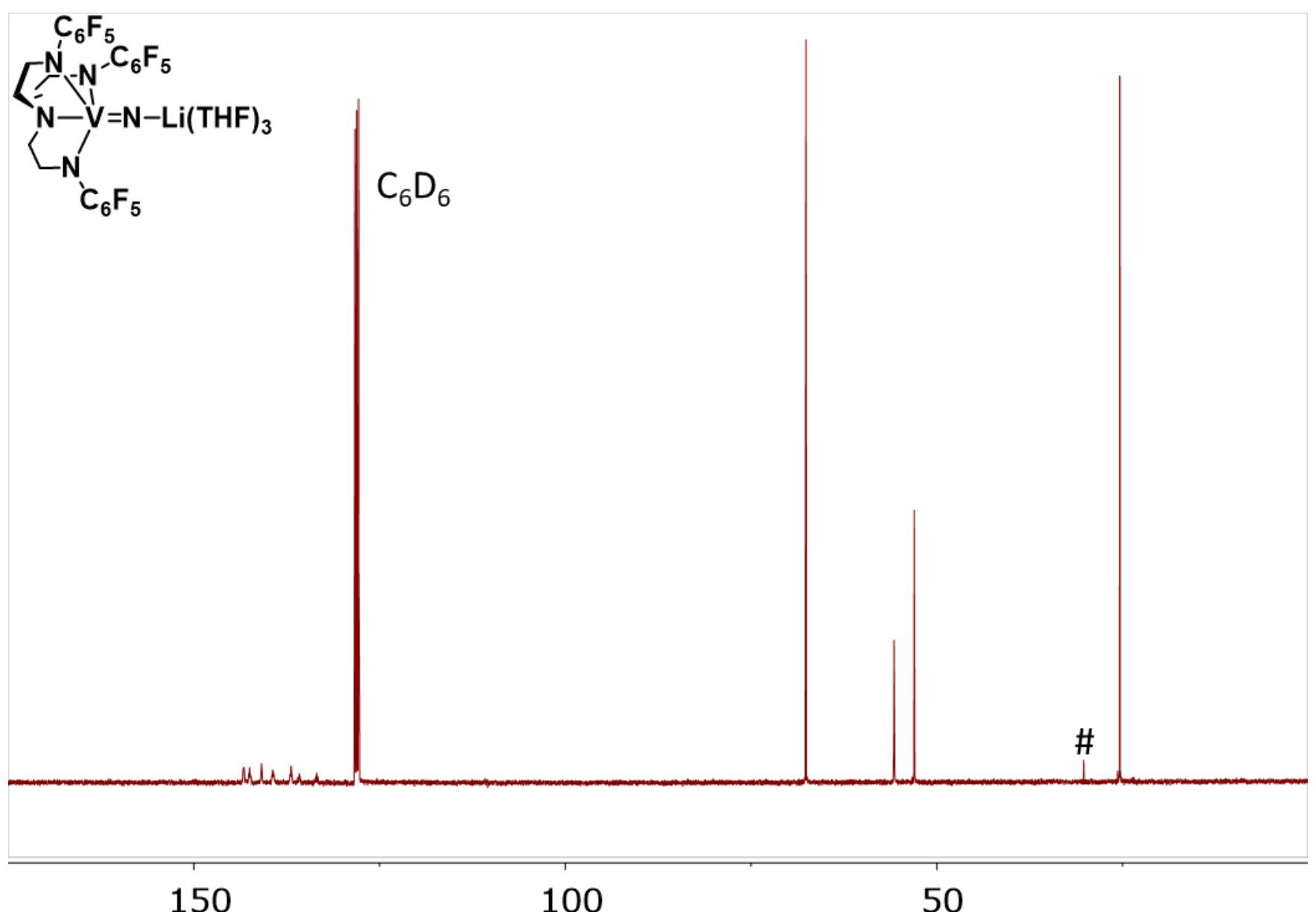

Figure S11. ${ }^{13} \mathrm{C}\left\{{ }^{1} \mathrm{H}\right\}$ NMR spectrum of (tren)VNLi $\left(100 \mathrm{MHz}, \mathrm{C}_{6} \mathrm{D}_{6}, 25{ }^{\circ} \mathrm{C}\right)$. (\# = pentane) 


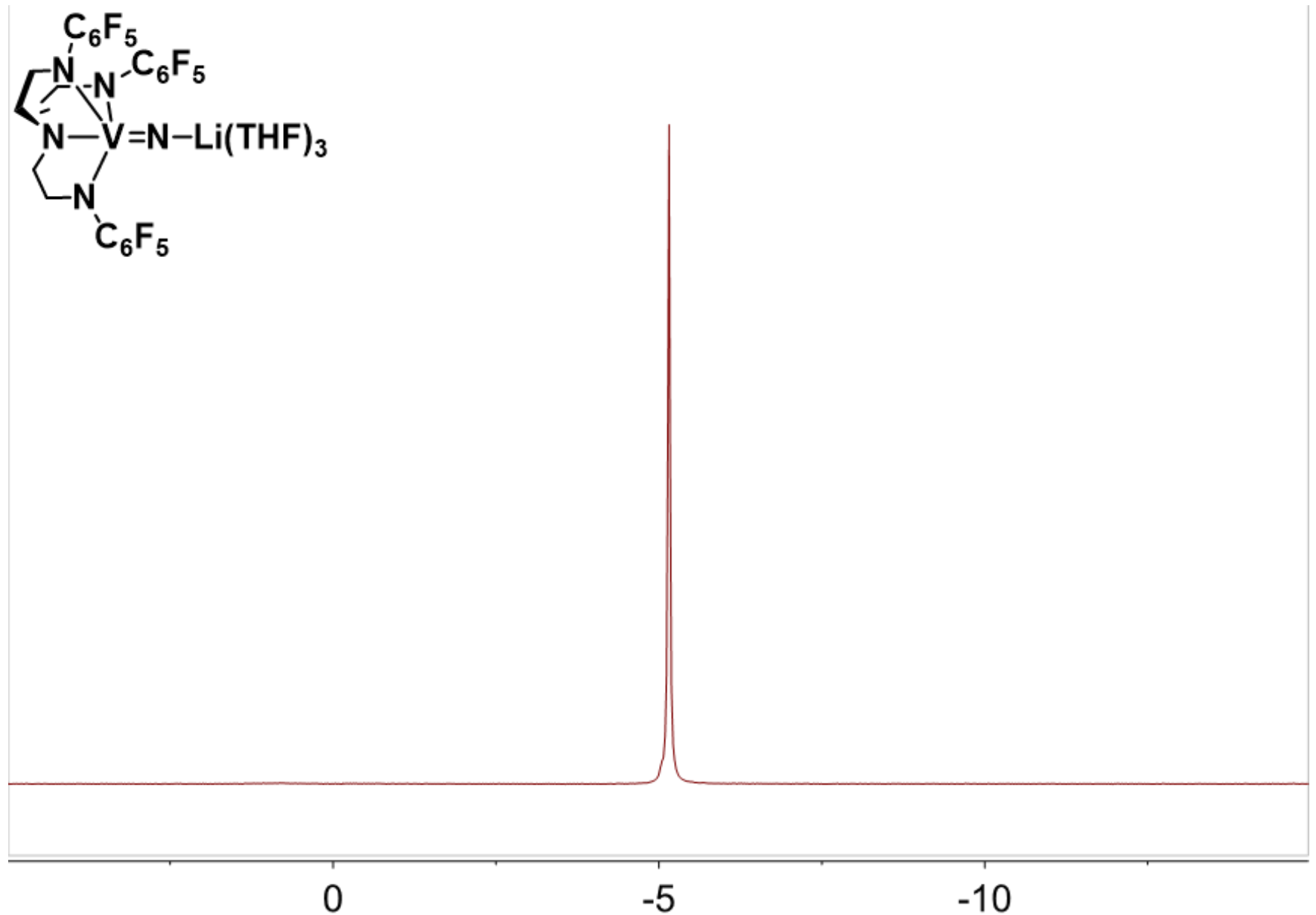

Figure S12. ${ }^{7} \mathrm{Li}\left\{{ }^{1} \mathrm{H}\right\}$ NMR spectrum of (tren)VNLi $\left(155 \mathrm{MHz}, \mathrm{C}_{6} \mathrm{D}_{6}, 25{ }^{\circ} \mathrm{C}\right)$. 

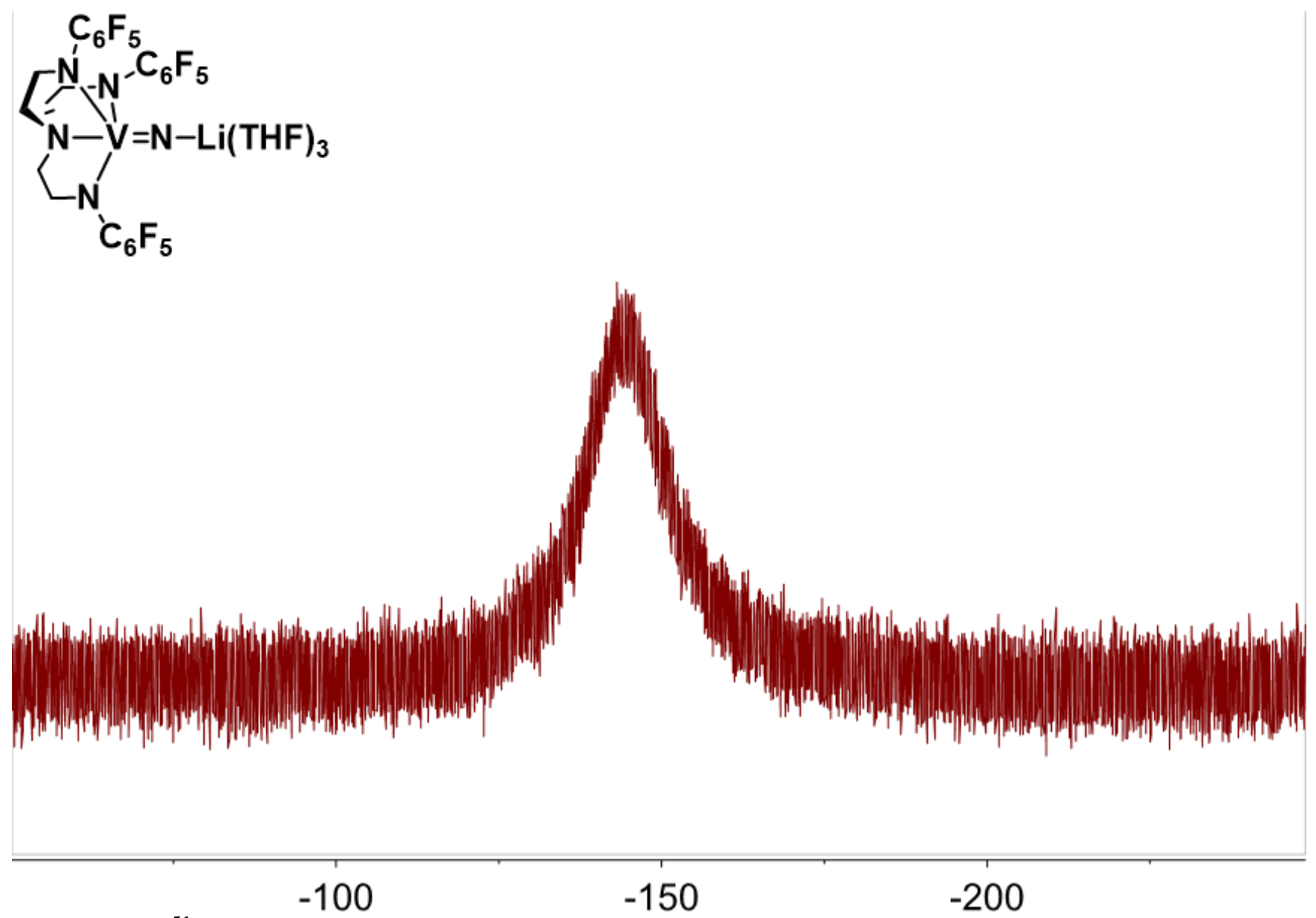

Figure S13. ${ }^{51} \mathrm{~V}$ NMR spectrum of (tren)VNLi $\left(105 \mathrm{MHz}, \mathrm{C}_{6} \mathrm{D}_{6}, 25{ }^{\circ} \mathrm{C}\right)$. 


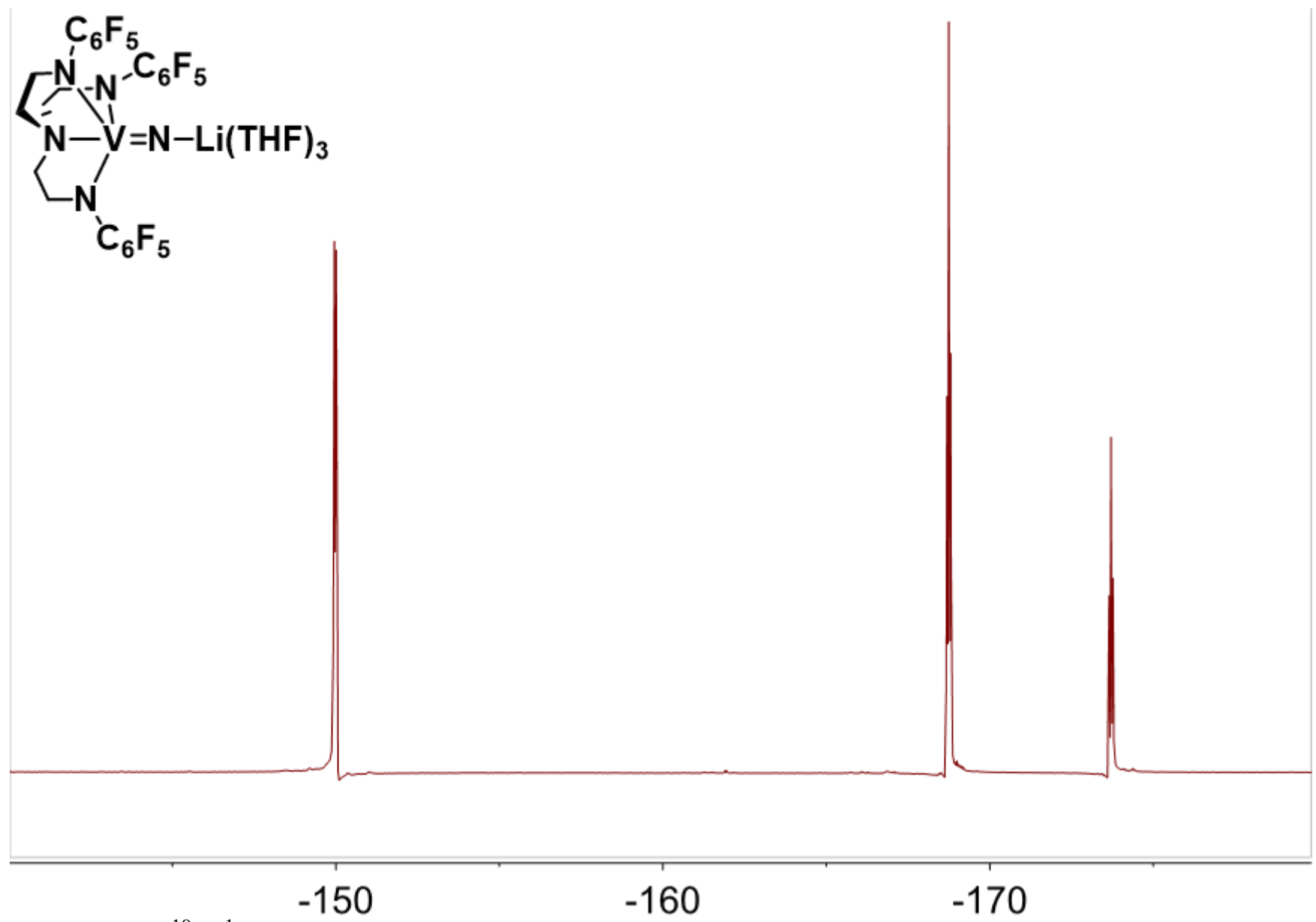

Figure S14. ${ }^{19} \mathrm{~F}\left\{{ }^{1} \mathrm{H}\right\}$ NMR spectrum of (tren)VNLi $\left(376 \mathrm{MHz}, \mathrm{C}_{6} \mathrm{D}_{6}, 25{ }^{\circ} \mathrm{C}\right)$. 


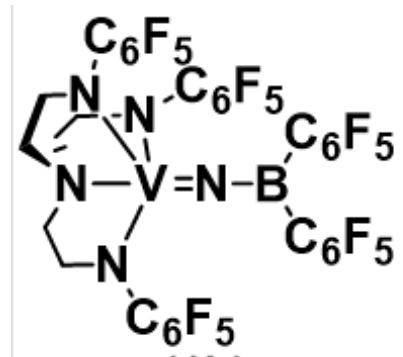

(1b)

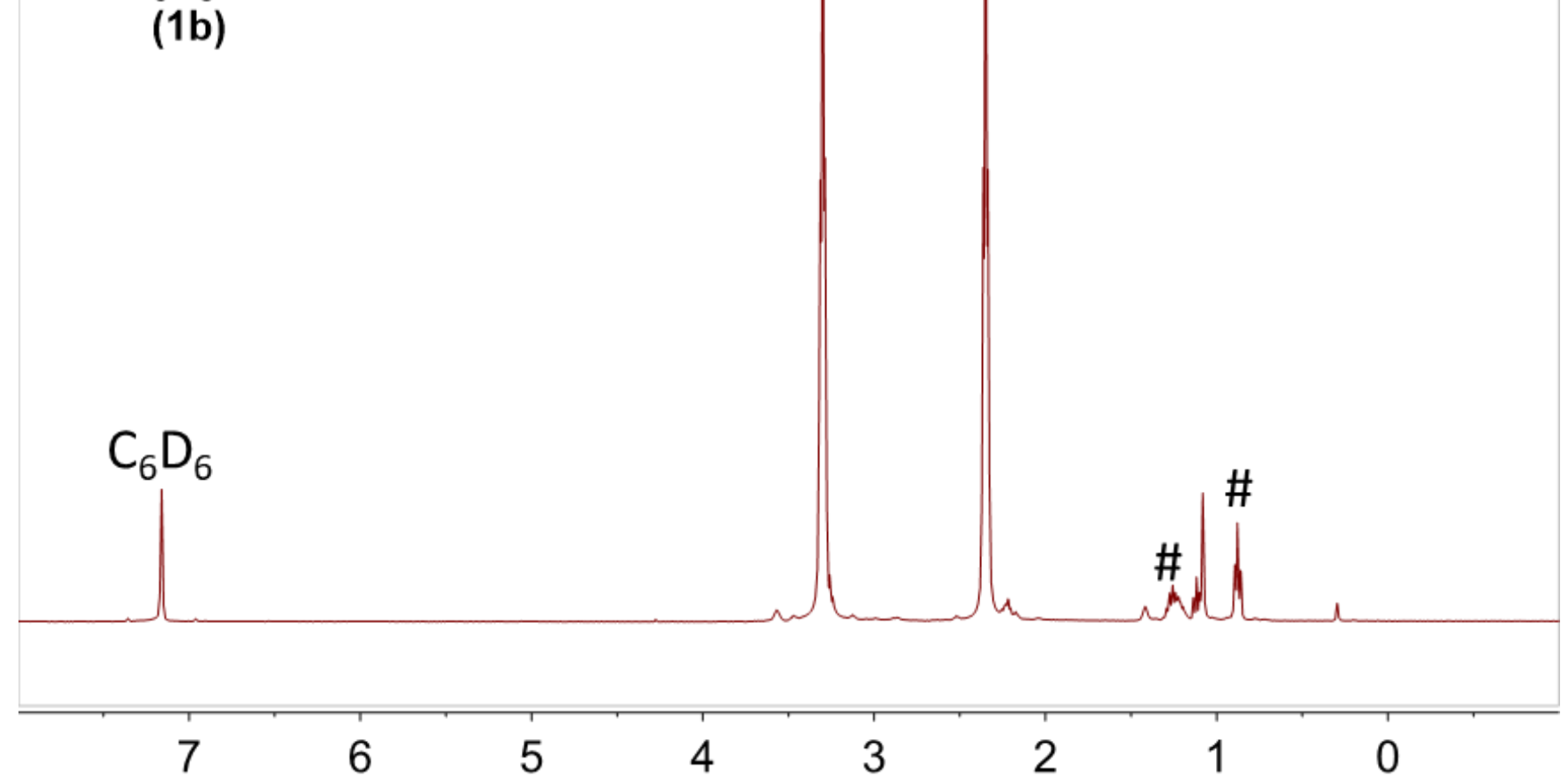

Figure S15. ${ }^{1} \mathrm{H}$ NMR of $\mathbf{1 b}\left(400 \mathrm{MHz}, \mathrm{C}_{6} \mathrm{D}_{6}, 25^{\circ} \mathrm{C}\right)$. (\# = pentane) 


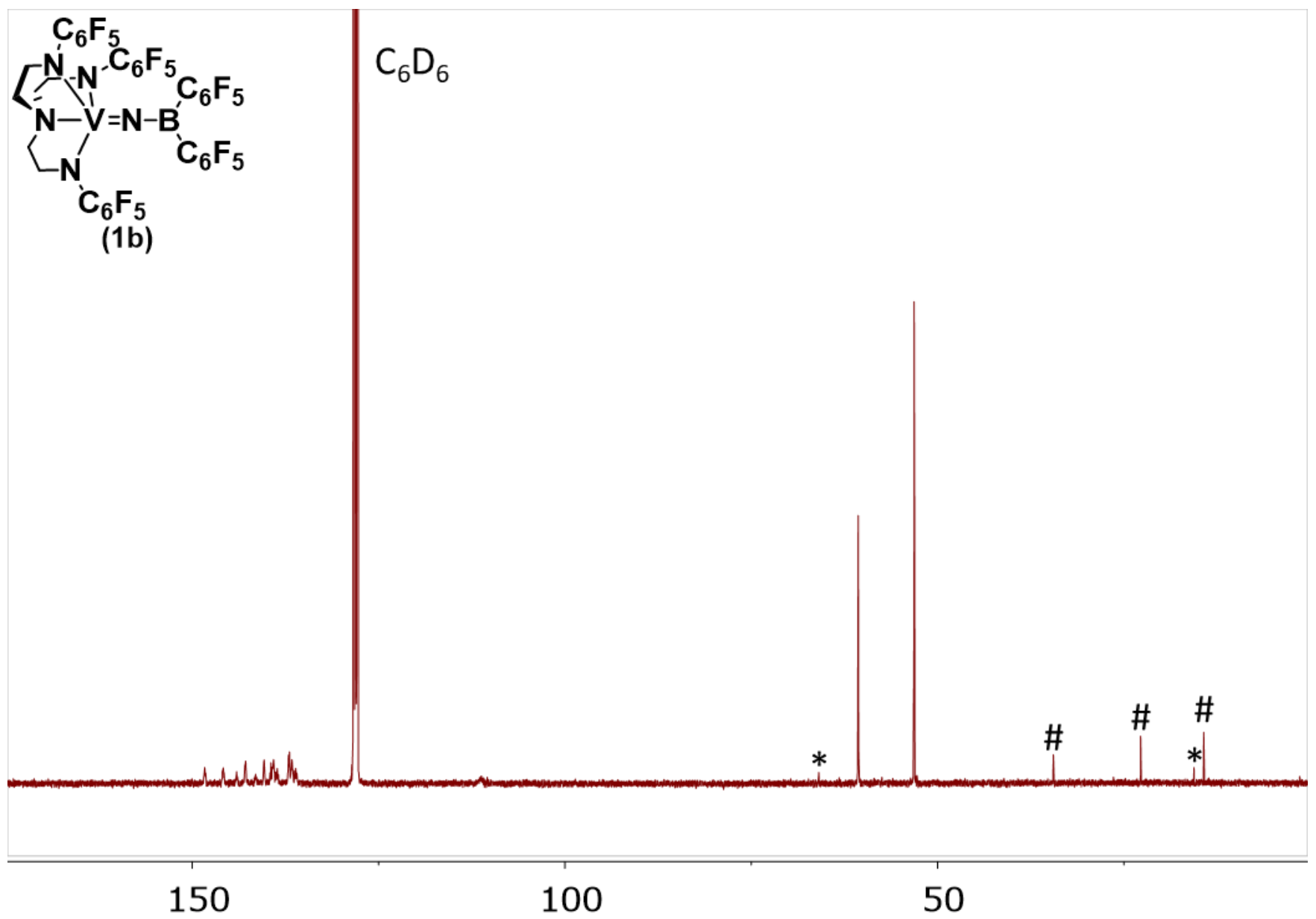

Figure S16. ${ }^{13} \mathrm{C}\left\{{ }^{1} \mathrm{H}\right\}$ NMR spectrum of $\mathbf{1 b}\left(100 \mathrm{MHz}, \mathrm{C}_{6} \mathrm{D}_{6}, 25{ }^{\circ} \mathrm{C}\right) .(\#=$ pentane, $*$ = ether $)$ 


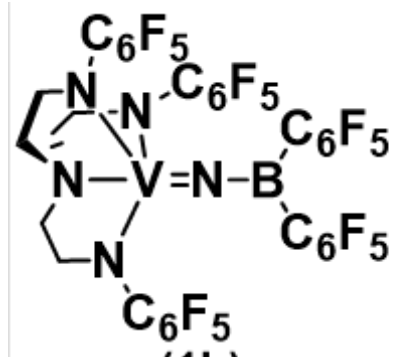

(1b)

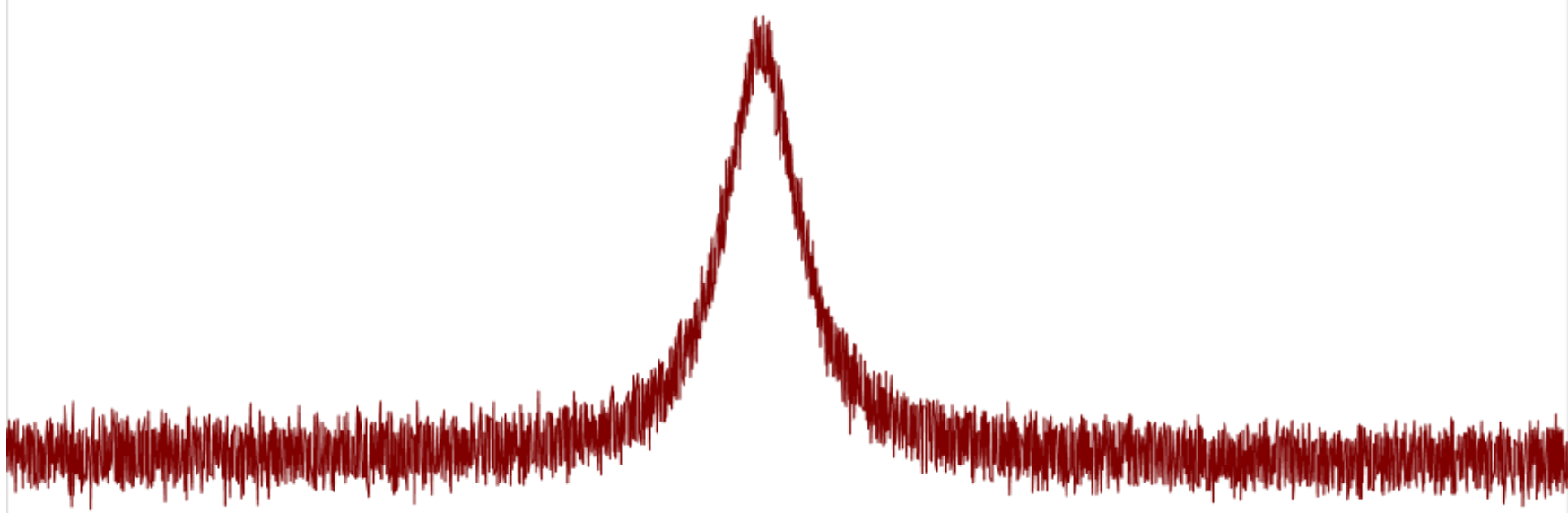

$-50 \quad-60$

$-70$

$-80$

$-90$

$-100$

$-110$

Figure S17. ${ }^{51} \mathrm{~V}$ NMR spectrum of $\mathbf{1 b}\left(105 \mathrm{MHz}, \mathrm{C}_{6} \mathrm{D}_{6}, 25^{\circ} \mathrm{C}\right)$. 


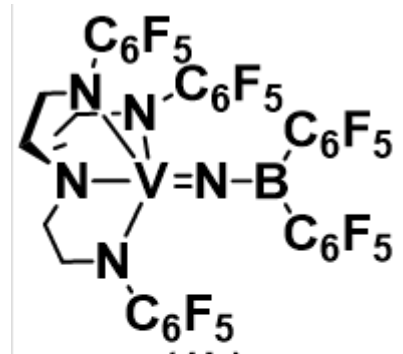

(1b)

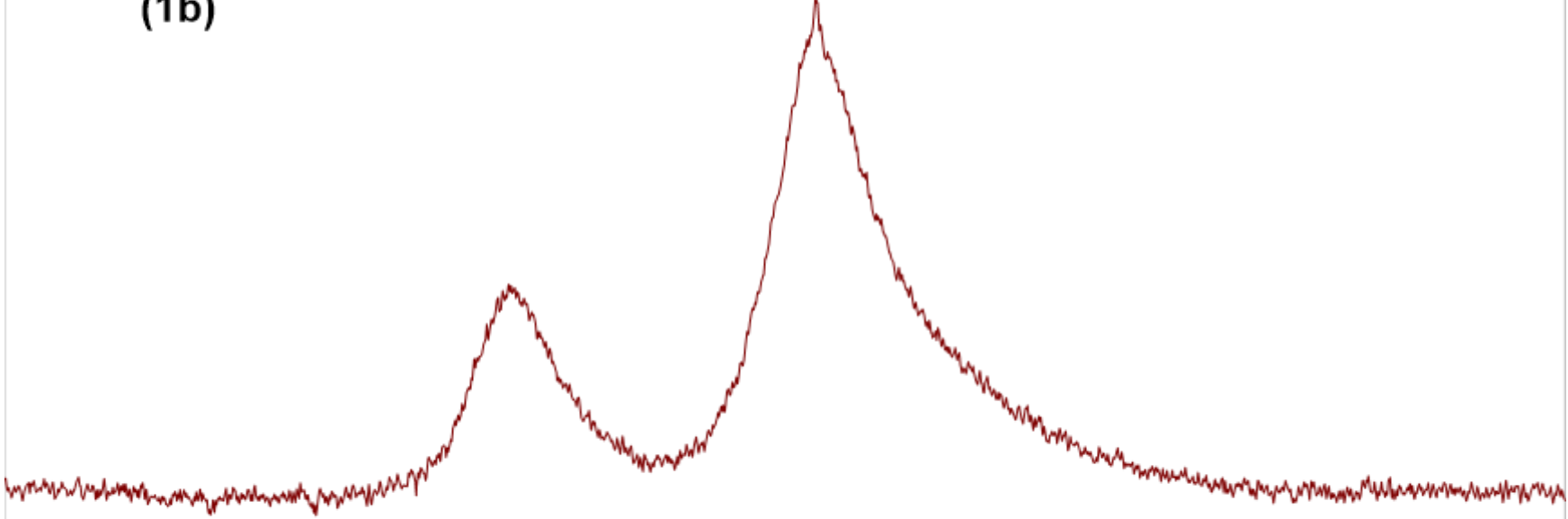

50

0

$-50$

Figure S18. ${ }^{11} \mathrm{~B}\left\{{ }^{1} \mathrm{H}\right\}$ NMR spectrum of $\mathbf{1 b}\left(128 \mathrm{MHz}, \mathrm{C}_{6} \mathrm{D}_{6}, 25{ }^{\circ} \mathrm{C}\right) .(\#=$ borosilicate resonance from NMR tube) 


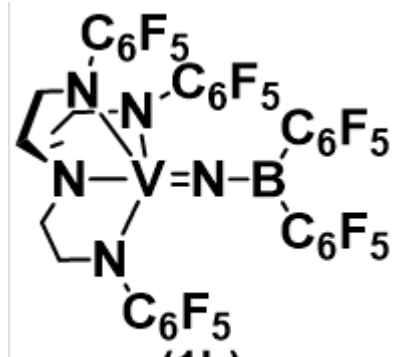

(1b)

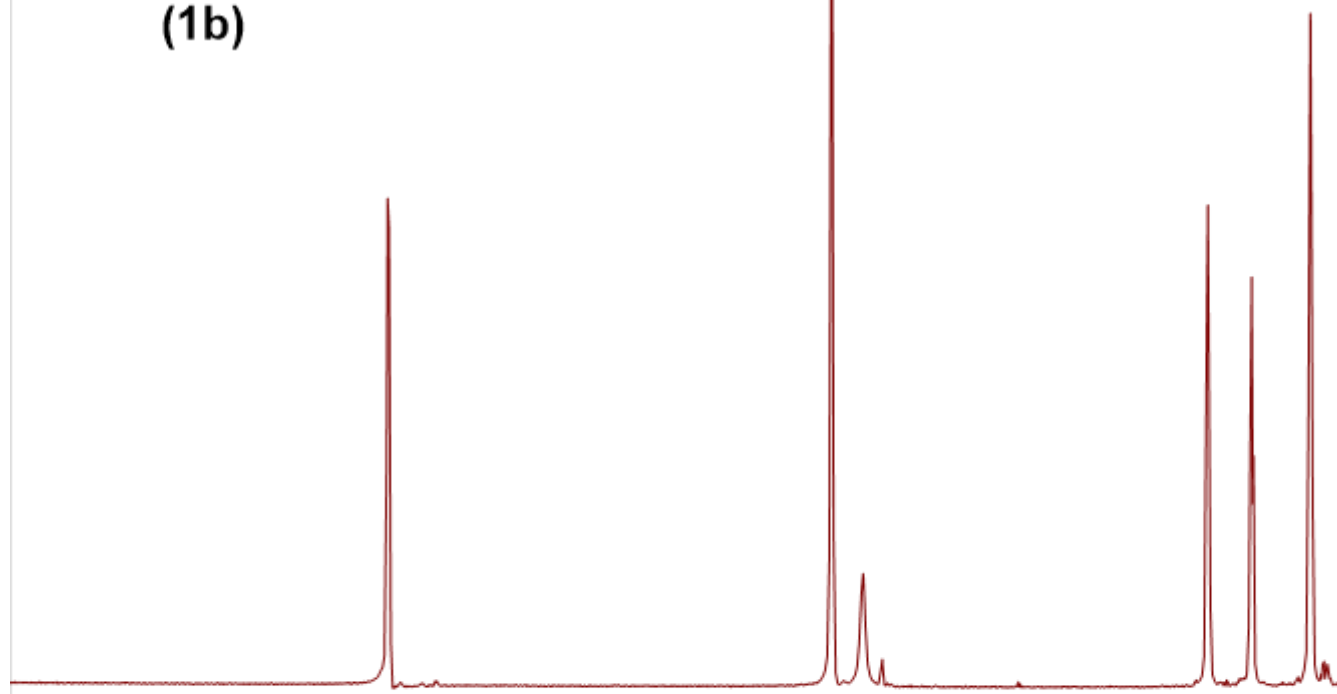

$$
-130
$$

$-160$

$-170$

Figure S19. ${ }^{19} \mathrm{~F}\left\{{ }^{1} \mathrm{H}\right\}$ NMR spectrum of $\mathbf{1 b}\left(376 \mathrm{MHz}, \mathrm{C}_{6} \mathrm{D}_{6}, 25{ }^{\circ} \mathrm{C}\right)$. 

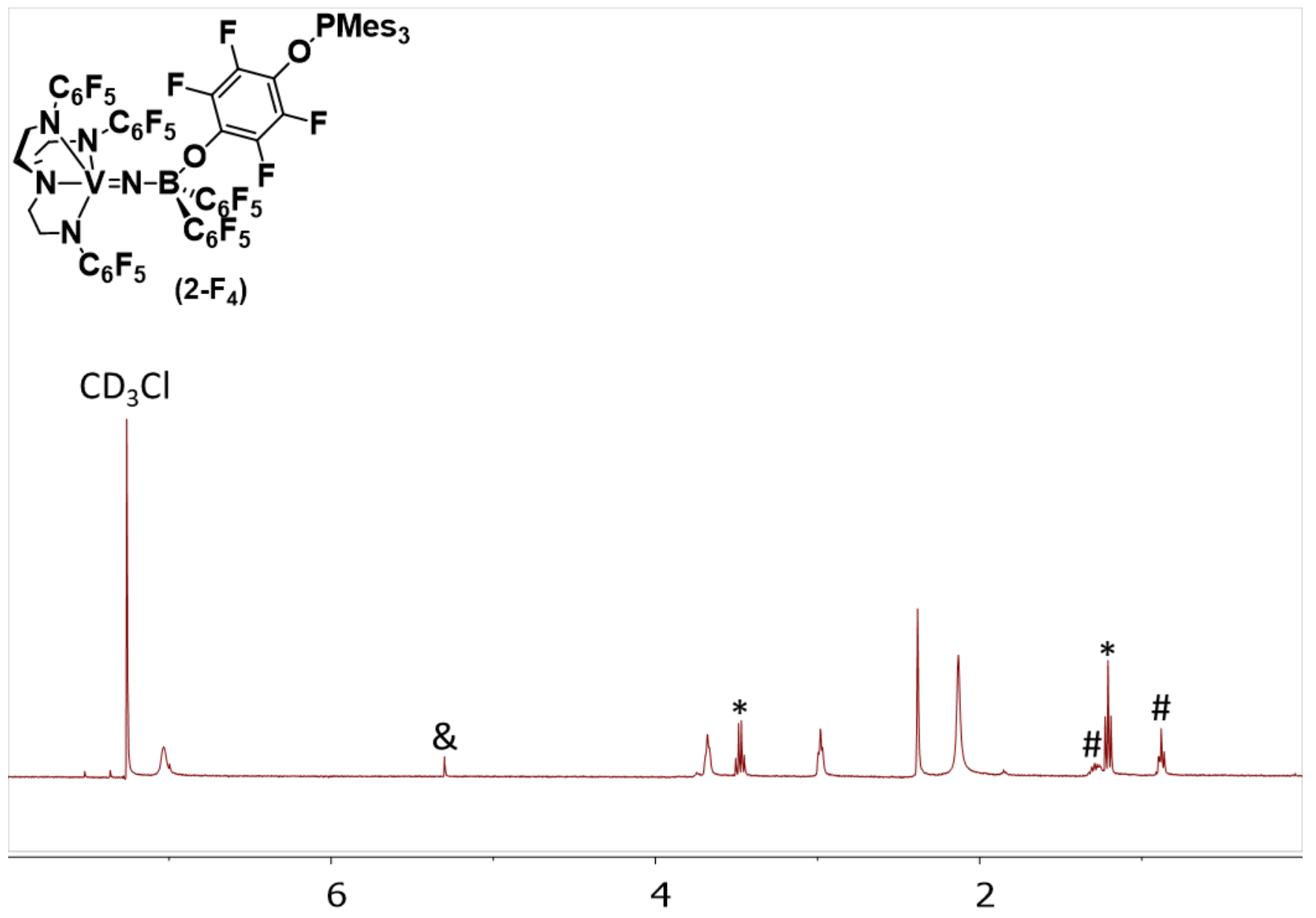

Figure S20. ${ }^{1} \mathrm{H}$ NMR spectrum of $\mathbf{2}-\mathbf{F}_{\mathbf{4}}\left(400 \mathrm{MHz}, \mathrm{CDCl}_{3}, 25^{\circ} \mathrm{C}\right)$. (\# = pentane, $*=$ ether, $\left.\&=\mathrm{DCM}\right)$ 


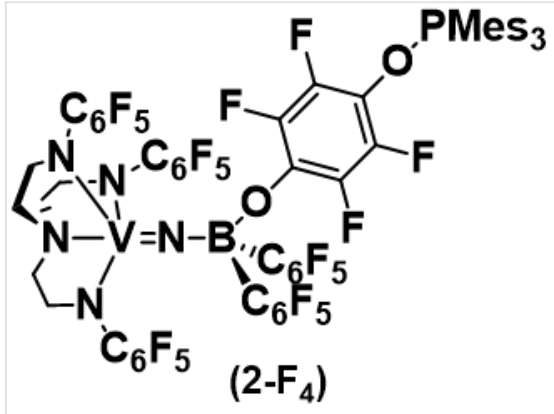

Figure S21. ${ }^{13} \mathrm{C}\left\{{ }^{1} \mathrm{H}\right\}$ NMR spectrum of $\mathbf{2}-\mathbf{F}_{4}\left(126 \mathrm{MHz}, \mathrm{CDCl}_{3}, 25{ }^{\circ} \mathrm{C}\right) .(\#=$ pentane, * $=$ ether, \& $=$ DCM) 

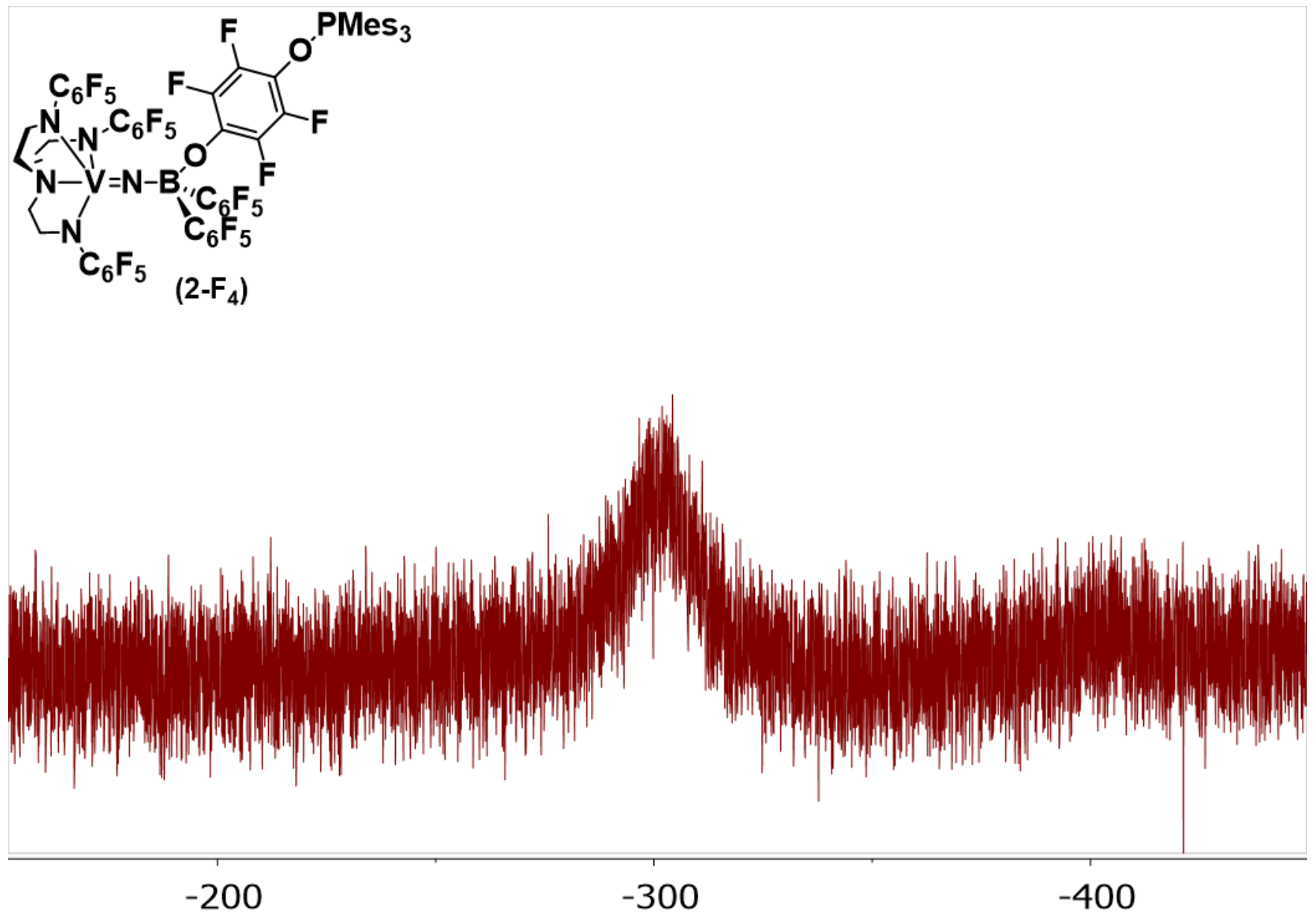

Figure S22. ${ }^{51} \mathrm{~V}$ NMR spectrum of $\mathbf{2}-\mathbf{F}_{4}\left(105 \mathrm{MHz}, \mathrm{CDCl}_{3}, 25{ }^{\circ} \mathrm{C}\right)$. 

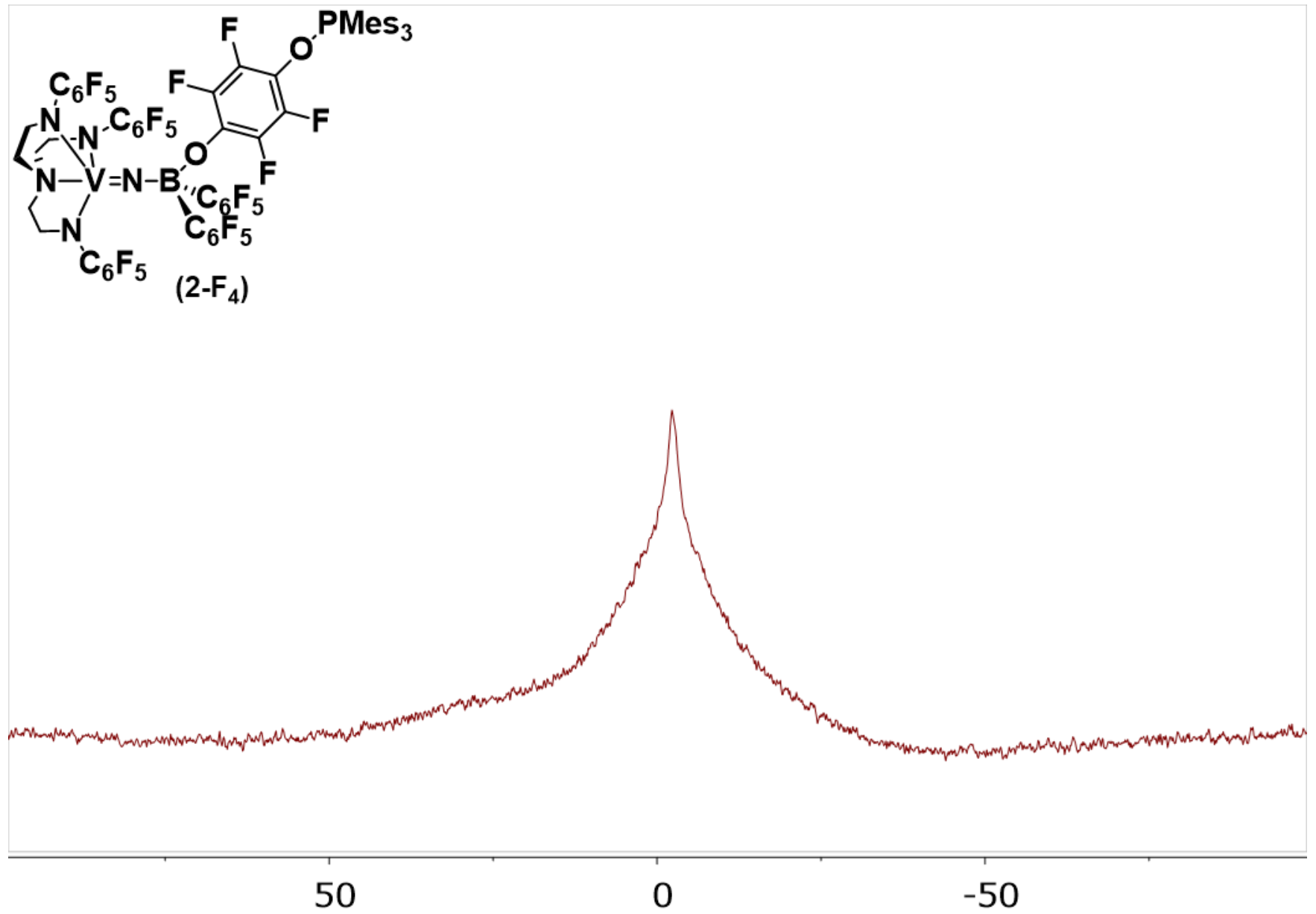

Figure S23. ${ }^{11} \mathrm{~B}\left\{{ }^{1} \mathrm{H}\right\}$ NMR spectrum of $\mathbf{2 - F _ { 4 }}\left(128 \mathrm{MHz}, \mathrm{CDCl}_{3}, 25{ }^{\circ} \mathrm{C}\right)$. 

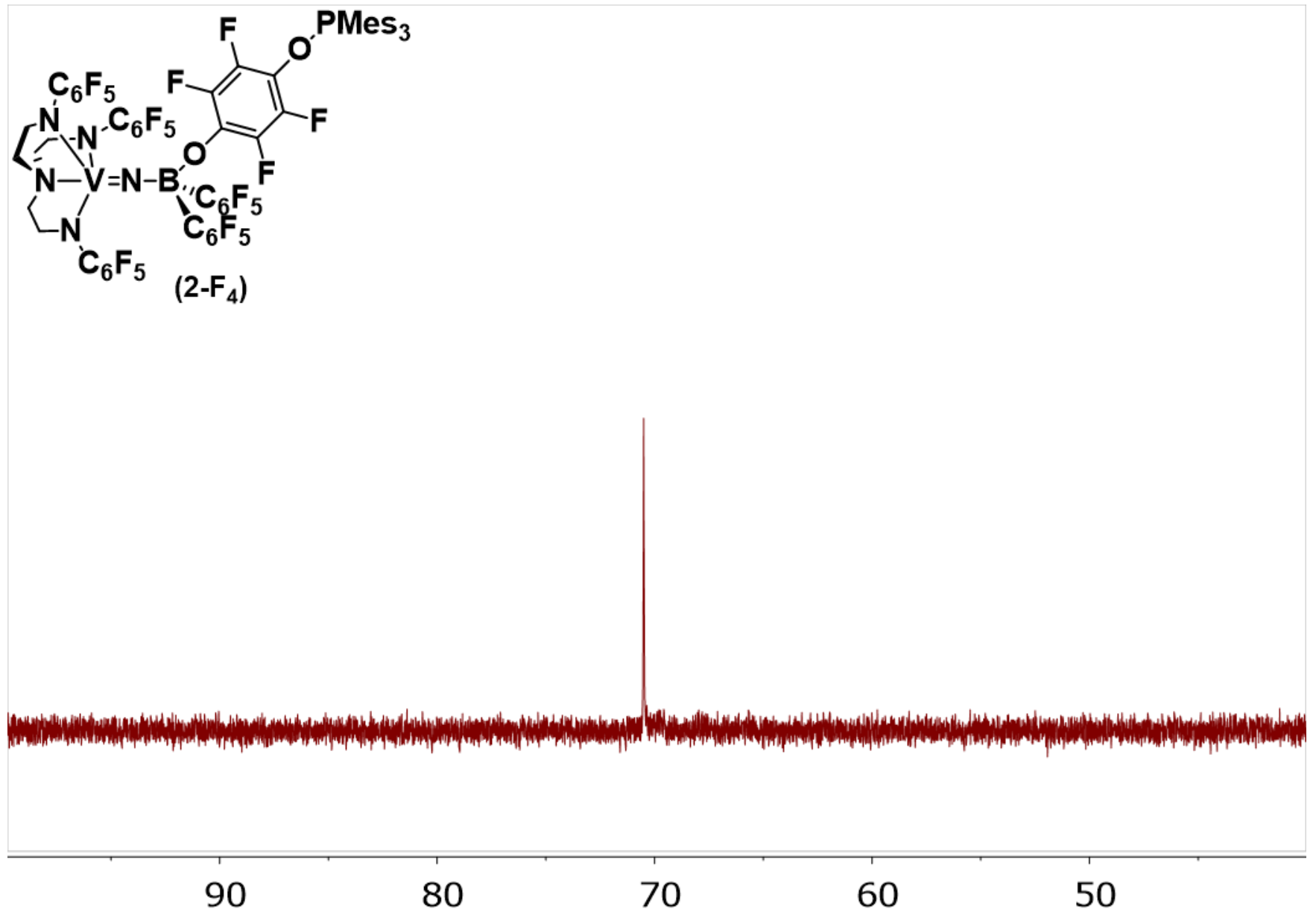

Figure S24. ${ }^{31} \mathrm{P}\left\{{ }^{1} \mathrm{H}\right\}$ NMR spectrum of $\mathbf{2}-\mathbf{F}_{\mathbf{4}}\left(162 \mathrm{MHz}, \mathrm{CDCl}_{3}, 25{ }^{\circ} \mathrm{C}\right)$. 

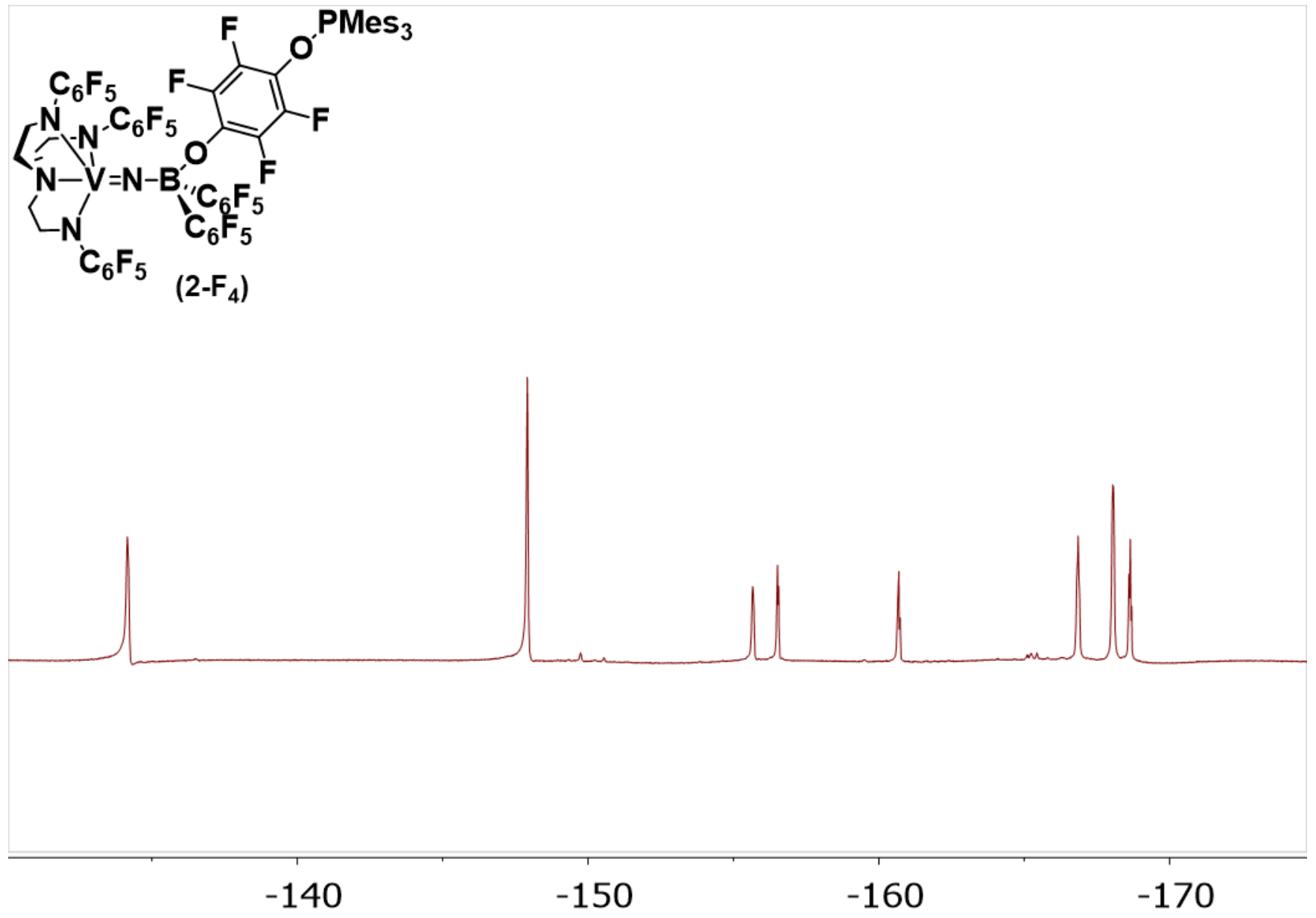

Figure S25. ${ }^{19} \mathrm{~F}\left\{{ }^{1} \mathrm{H}\right\}$ NMR spectrum of $\mathbf{2}-\mathbf{F}_{\mathbf{4}}\left(376 \mathrm{MHz}, \mathrm{CDCl}_{3}, 25{ }^{\circ} \mathrm{C}\right)$. 


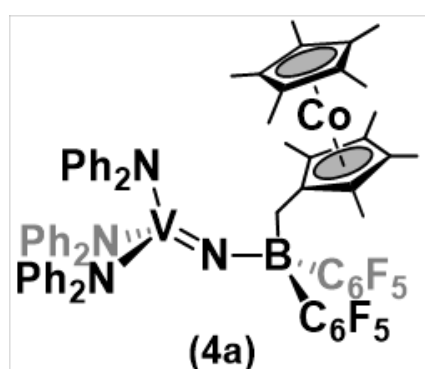

(4a)

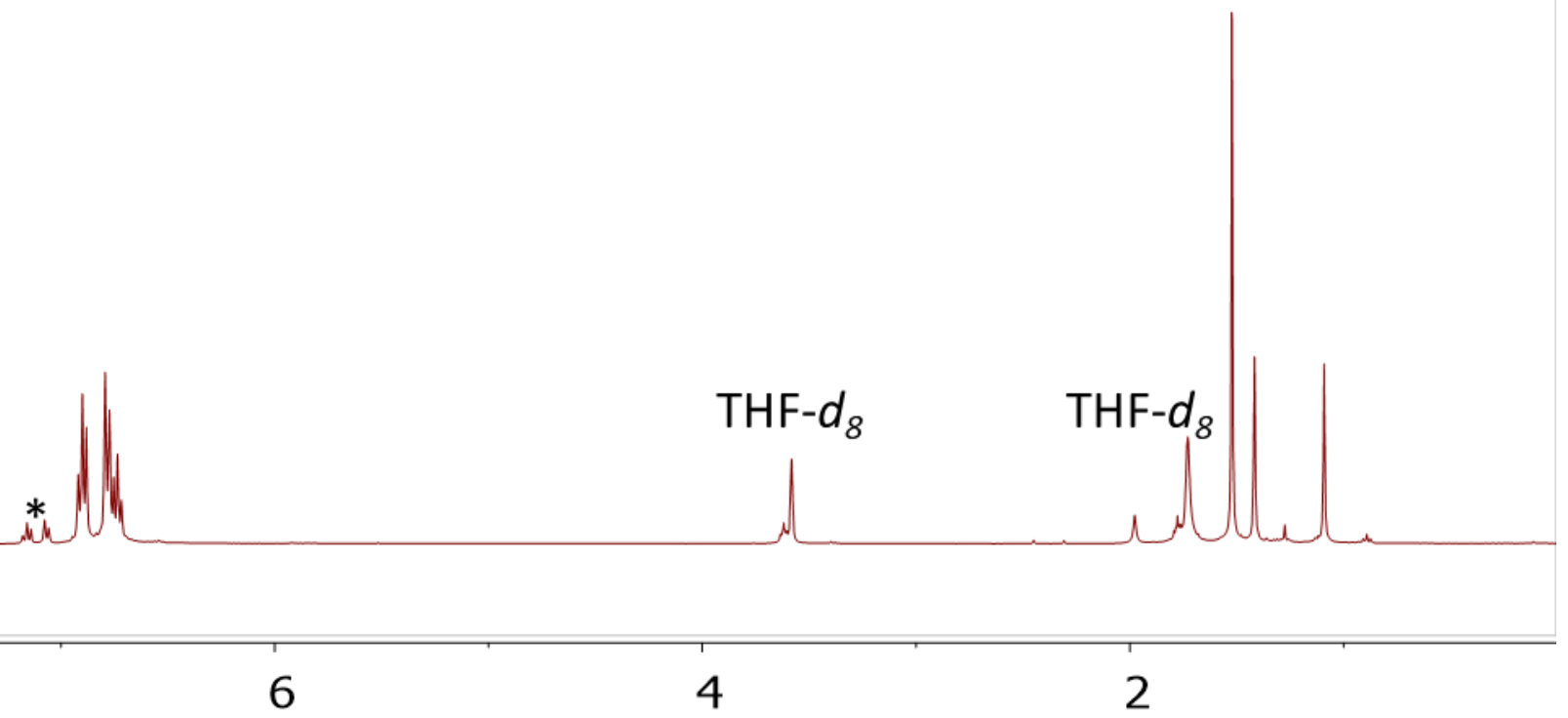

Figure S26. ${ }^{1} \mathrm{H}$ NMR of $4 \mathbf{a}\left(600 \mathrm{MHz}, \mathrm{THF}-d_{8}, 25{ }^{\circ} \mathrm{C}\right) .\left({ }^{*} \pi-\pi\right.$ stacked $\mathrm{C}_{6} \mathrm{H}_{5}$ ring $)$ 


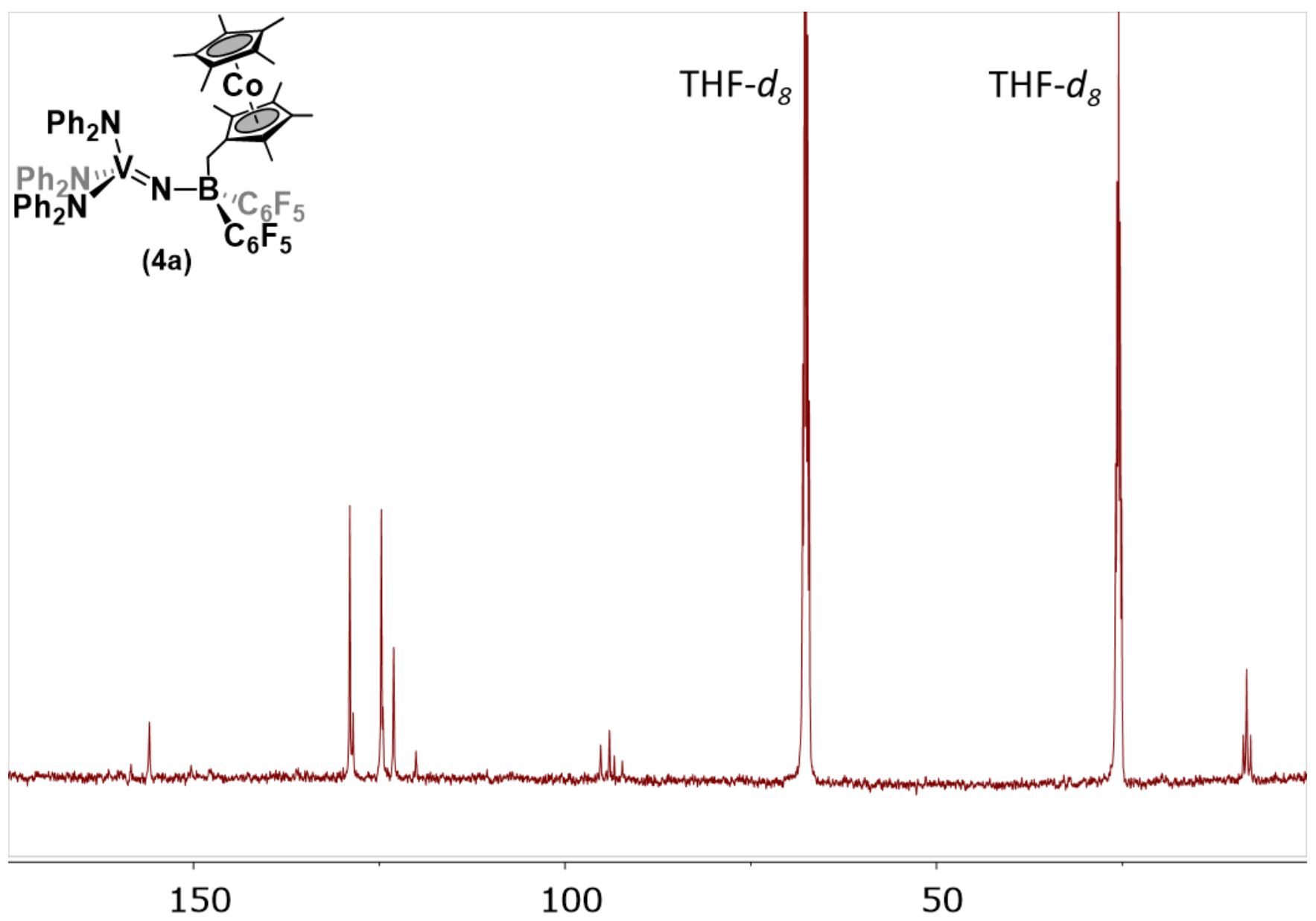

Figure S27. ${ }^{13} \mathrm{C}\left\{{ }^{1} \mathrm{H}\right\}$ NMR spectrum of $\mathbf{4 a}\left(100 \mathrm{MHz}, \mathrm{THF}-d 8,25{ }^{\circ} \mathrm{C}\right)$. 


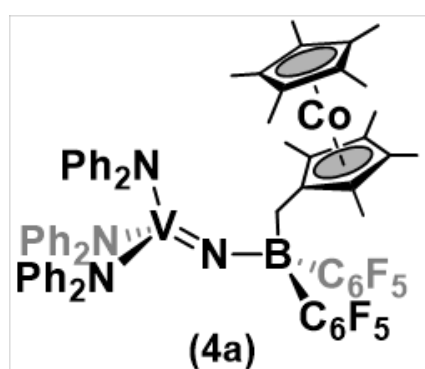

(4a)

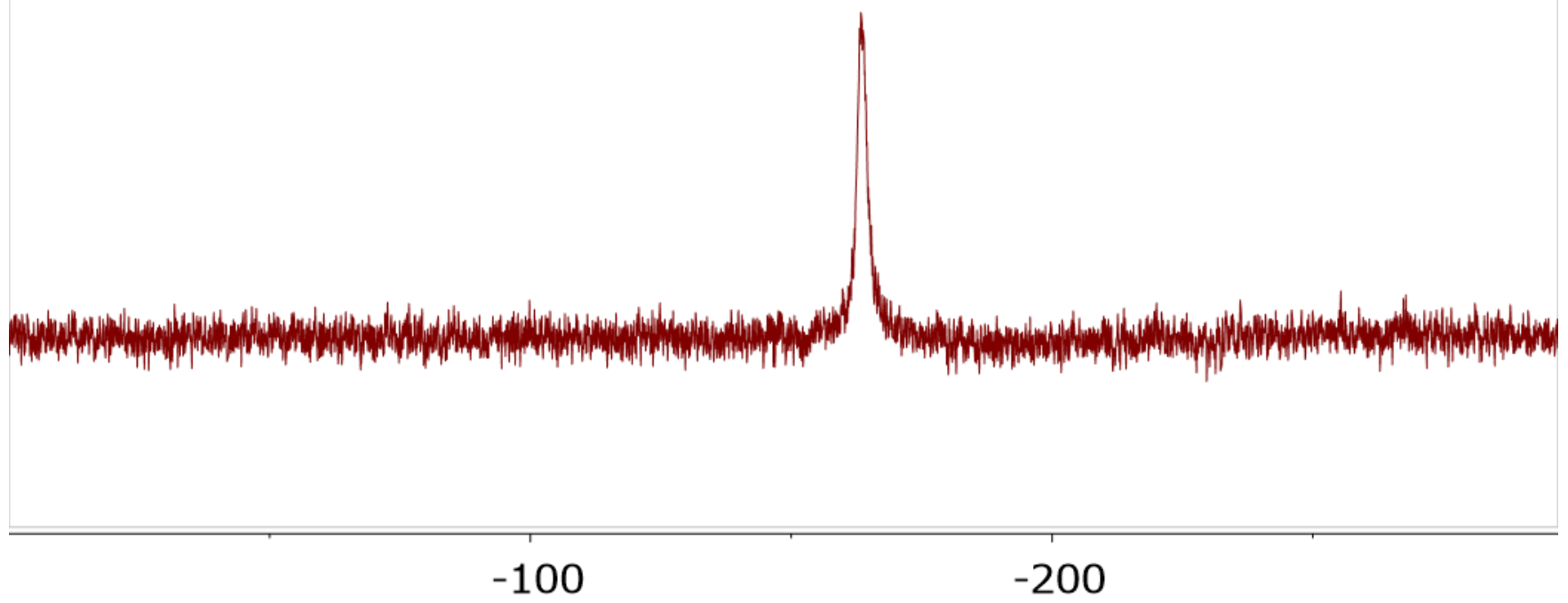

Figure S28. ${ }^{51} \mathrm{~V}$ NMR spectrum of $\mathbf{4 a}\left(105 \mathrm{MHz}\right.$, THF- $\left.d 8,25{ }^{\circ} \mathrm{C}\right)$. 


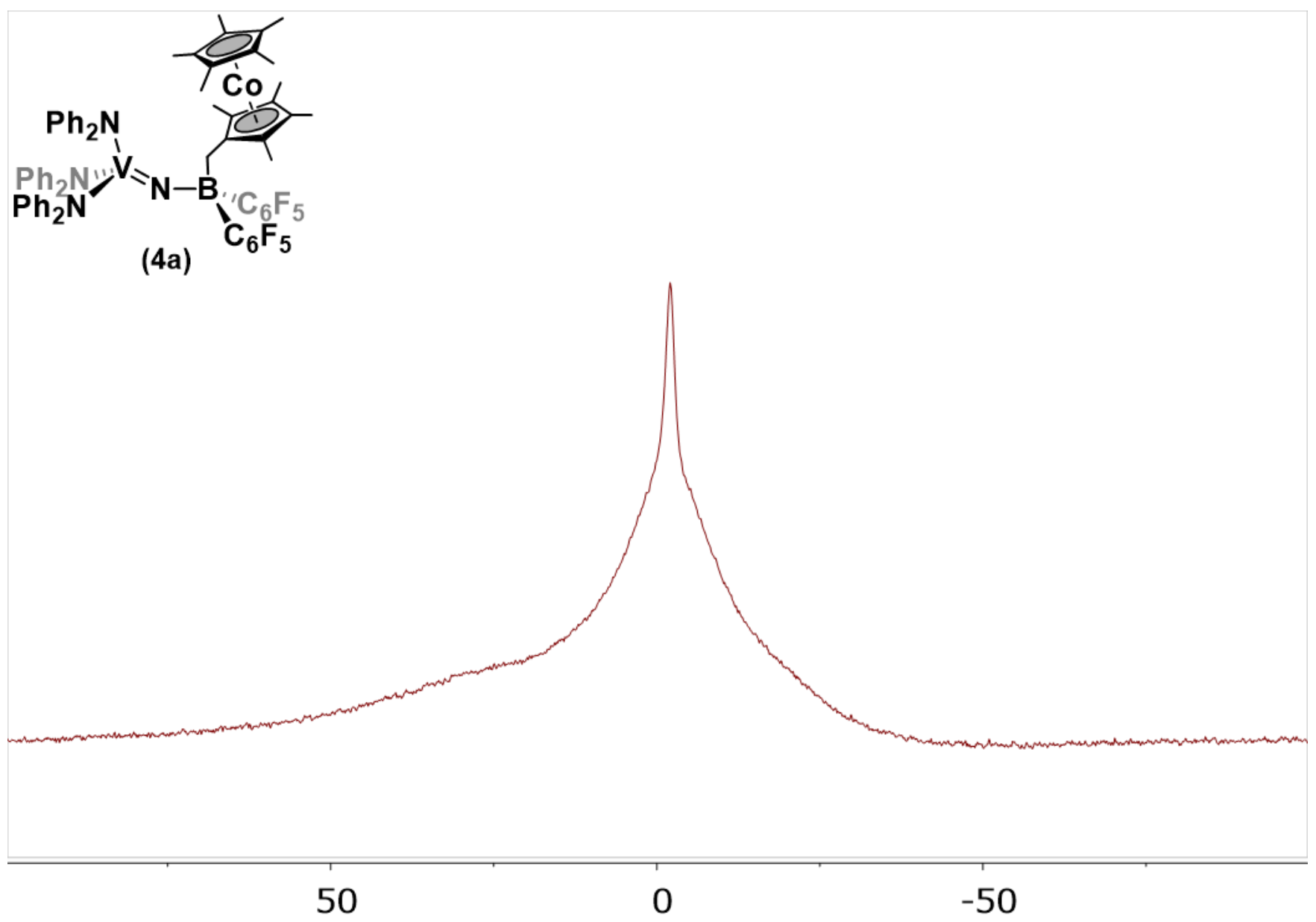

Figure S29. ${ }^{11} \mathrm{~B}\left\{{ }^{1} \mathrm{H}\right\}$ NMR spectrum of $4 \mathbf{a}\left(128 \mathrm{MHz}, \mathrm{THF}-d 8,25{ }^{\circ} \mathrm{C}\right)$. 


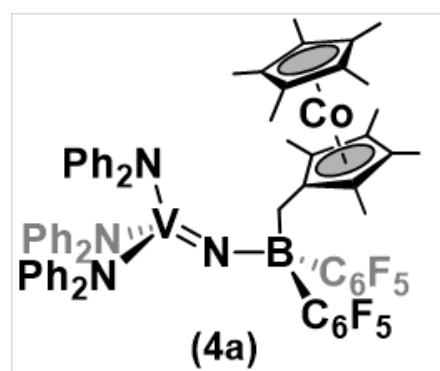

(4a)

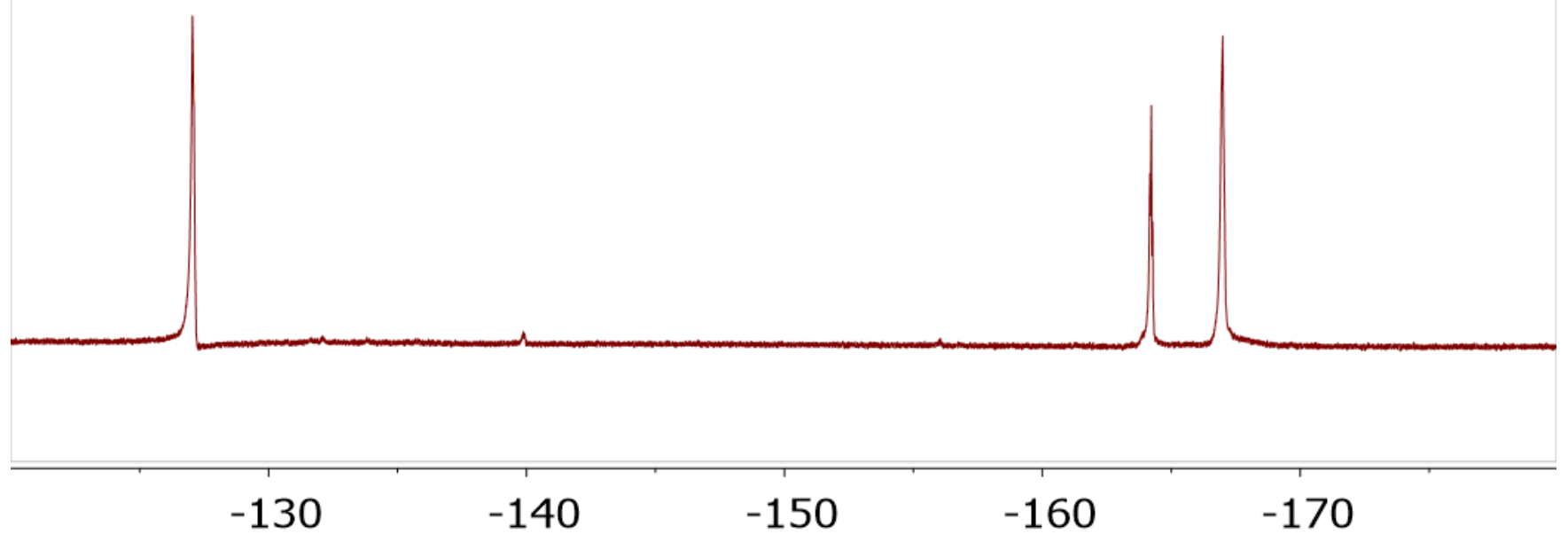

Figure S30. ${ }^{19} \mathrm{~F}\left\{{ }^{1} \mathrm{H}\right\}$ NMR spectrum of $\mathbf{4 a}\left(376 \mathrm{MHz}, \mathrm{THF}-d_{8}, 25{ }^{\circ} \mathrm{C}\right)$. 


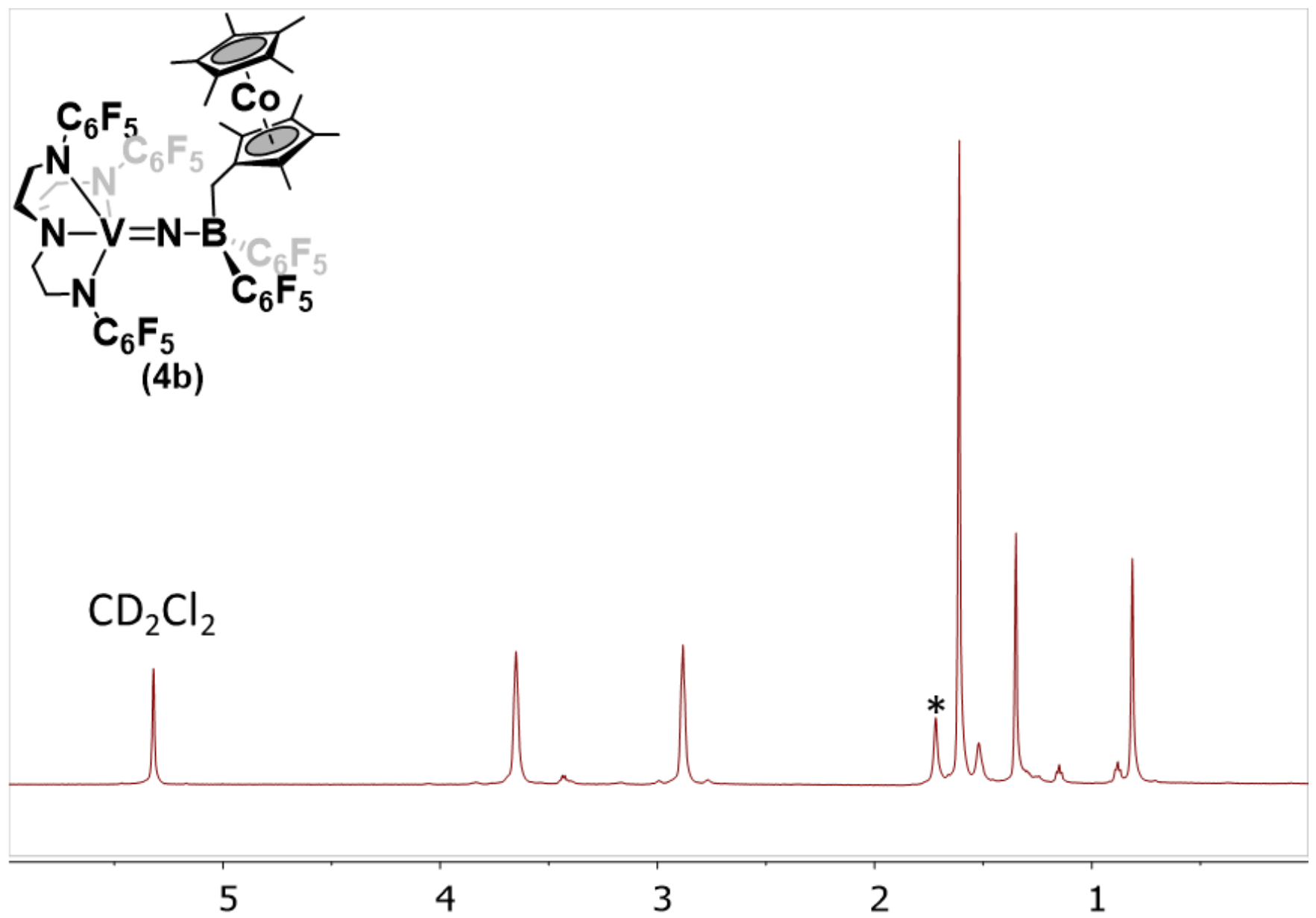

Figure S31. ${ }^{1} \mathrm{H}$ NMR of $\mathbf{4 b}\left(600 \mathrm{MHz}, \mathrm{CD}_{2} \mathrm{Cl}_{2}, 25^{\circ} \mathrm{C}\right) .(*$ unidentified resonance $)$ 


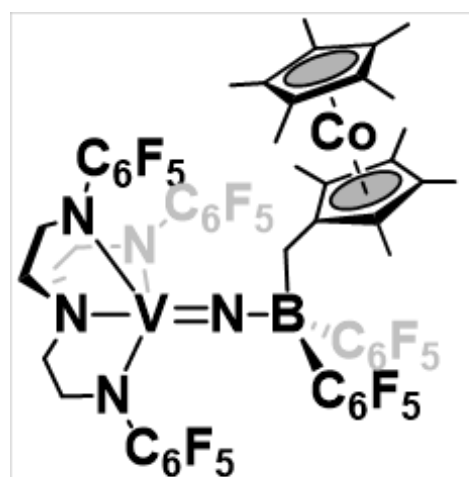

(4b)

\section{$\mathrm{CD}_{2} \mathrm{Cl}_{2}$}

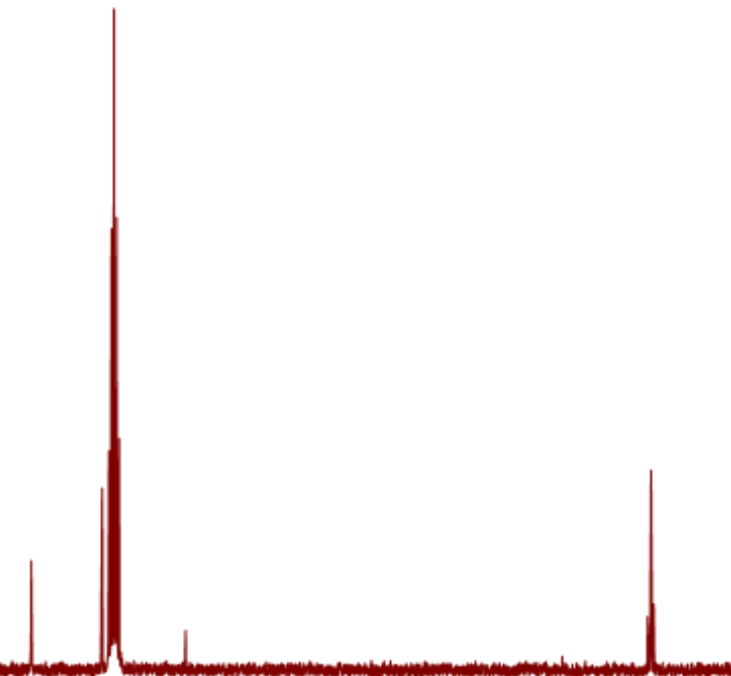

\section{0}

50

Figure S32. ${ }^{13} \mathrm{C}\left\{{ }^{1} \mathrm{H}\right\}$ NMR spectrum of $\mathbf{4 b}\left(100 \mathrm{MHz}, \mathrm{CD}_{2} \mathrm{Cl}_{2}, 25{ }^{\circ} \mathrm{C}\right)$. 


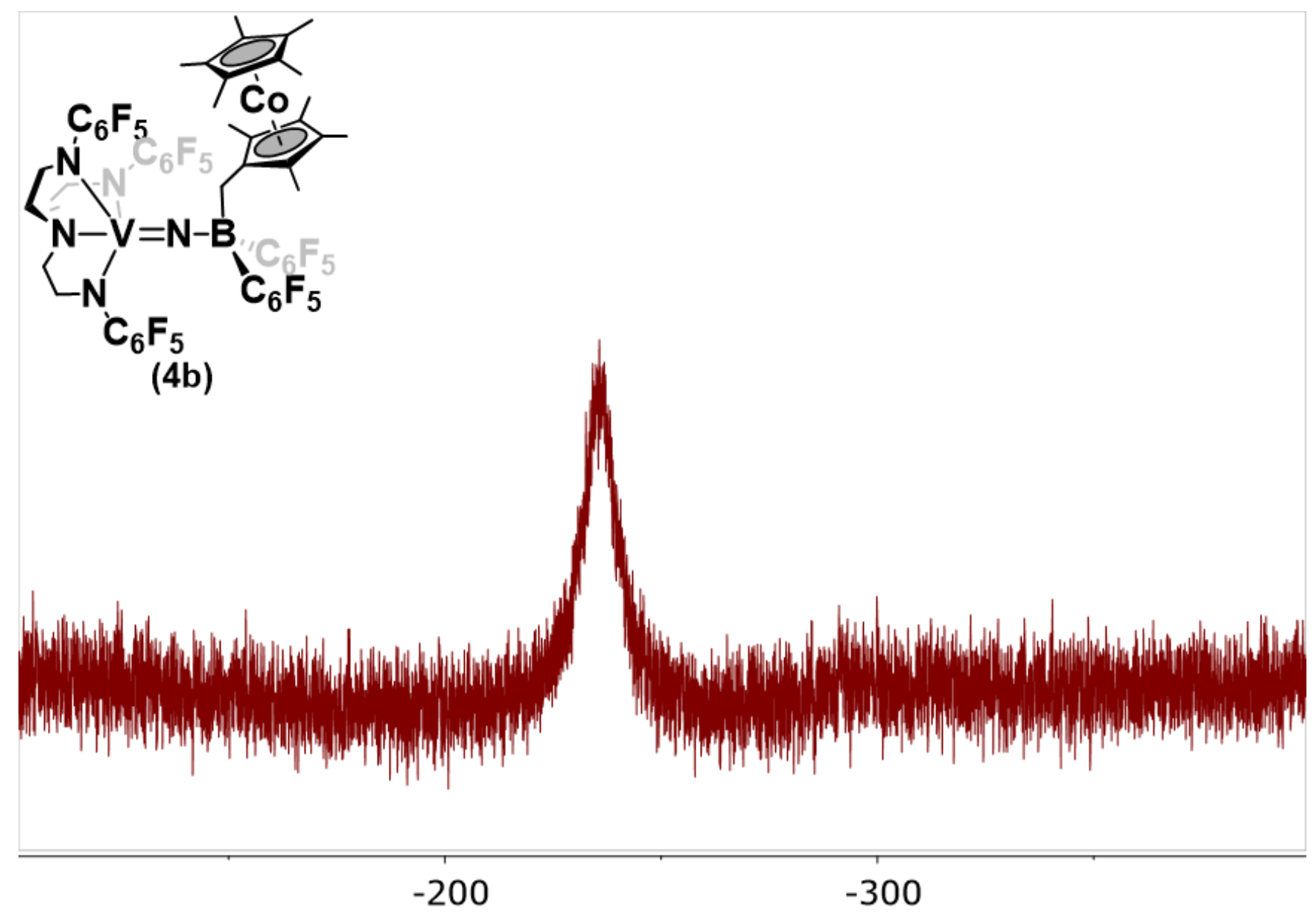

Figure S33. ${ }^{51} \mathrm{~V}$ NMR spectrum of $4 \mathbf{b}\left(105 \mathrm{MHz}, \mathrm{CD}_{2} \mathrm{Cl}_{2}, 25^{\circ} \mathrm{C}\right)$. 


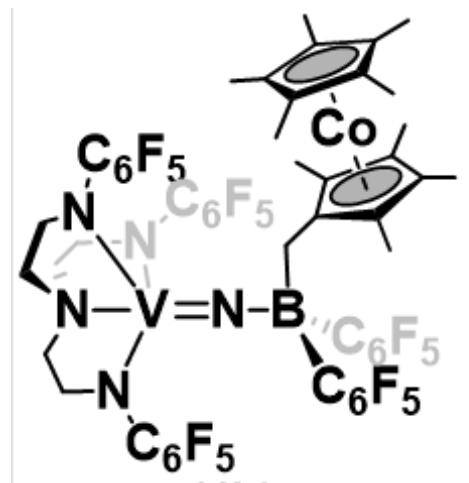

(4b)

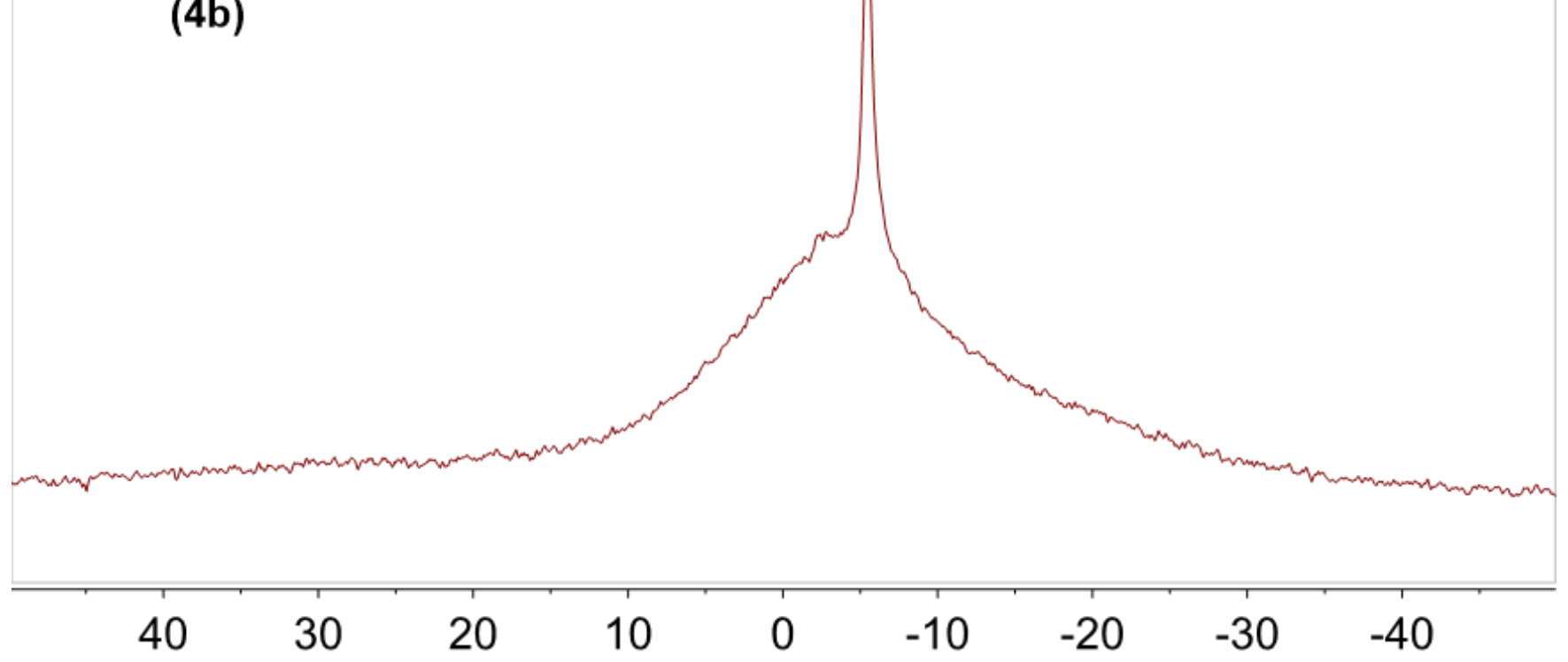

Figure S34. ${ }^{11} \mathrm{~B}\left\{{ }^{1} \mathrm{H}\right\}$ NMR spectrum of $\mathbf{4 b}\left(128 \mathrm{MHz}, \mathrm{CD}_{2} \mathrm{Cl}_{2}, 25{ }^{\circ} \mathrm{C}\right)$. 


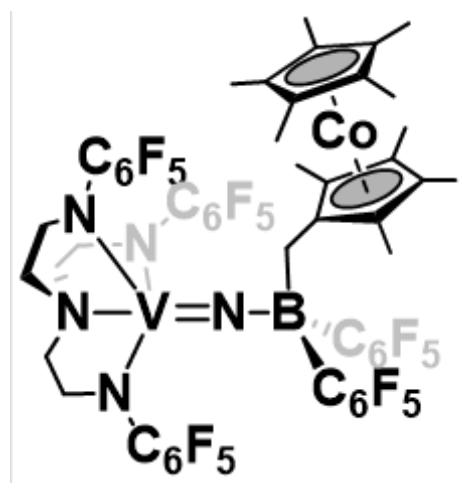

(4b)

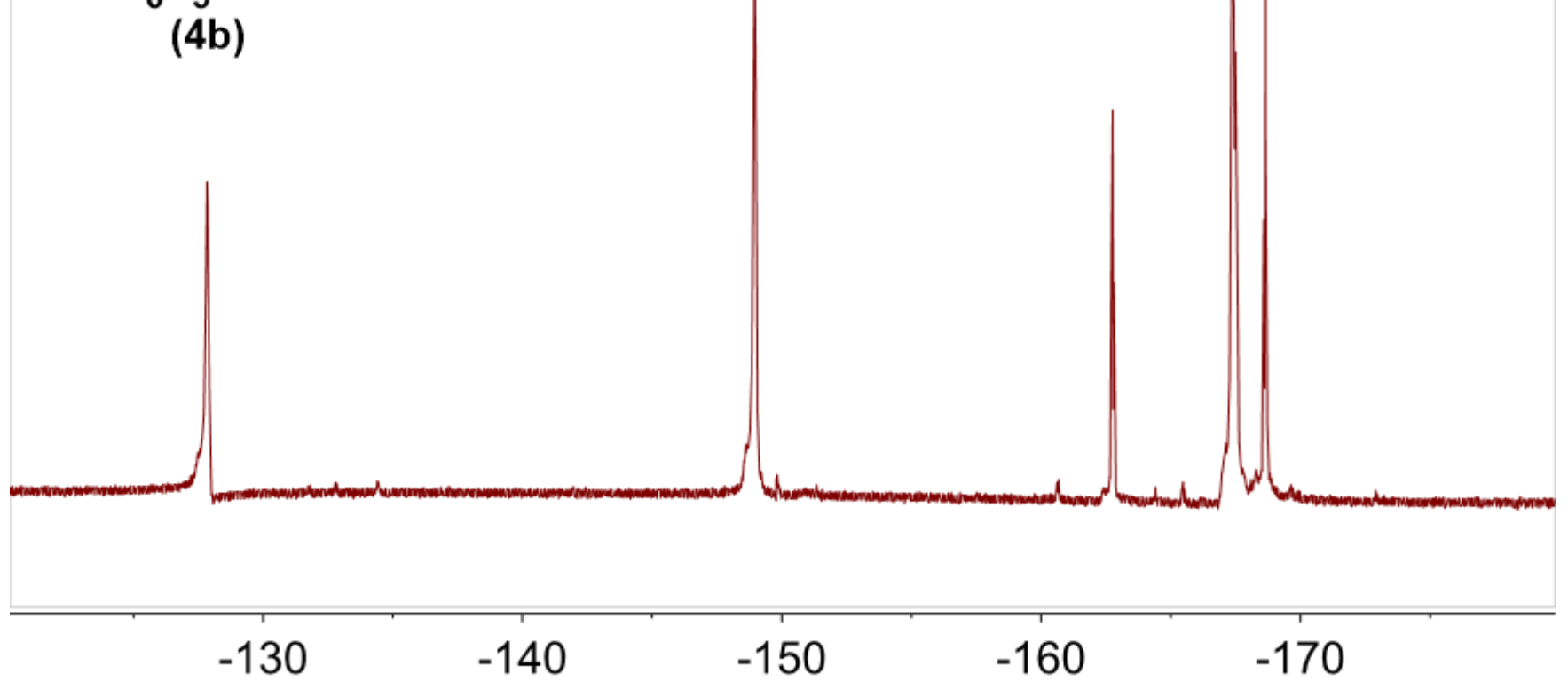

Figure S35. ${ }^{19} \mathrm{~F}\left\{{ }^{1} \mathrm{H}\right\}$ NMR spectrum of $\mathbf{4 b}\left(376 \mathrm{MHz}, \mathrm{CD}_{2} \mathrm{Cl}_{2}, 25{ }^{\circ} \mathrm{C}\right)$. 


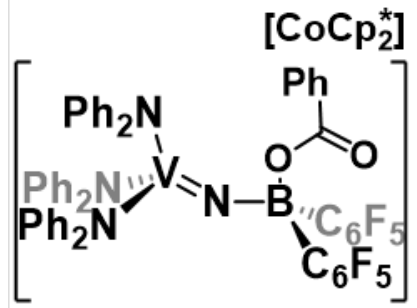

(5a)

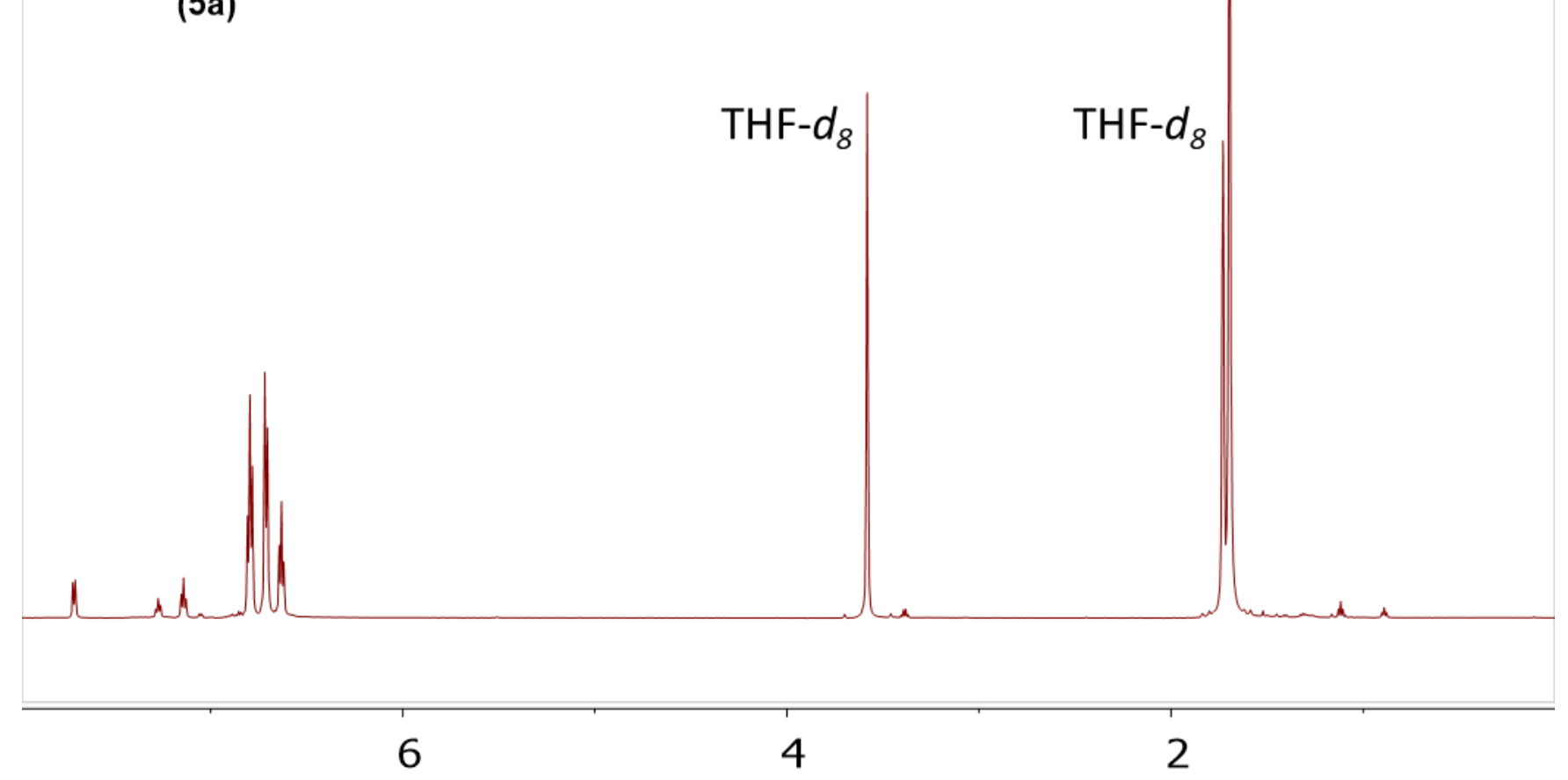

Figure S36. ${ }^{1} \mathrm{H}$ NMR spectrum of $\mathbf{5 a}\left(600 \mathrm{MHz}, \mathrm{THF}-d 8,25{ }^{\circ} \mathrm{C}\right)$. 


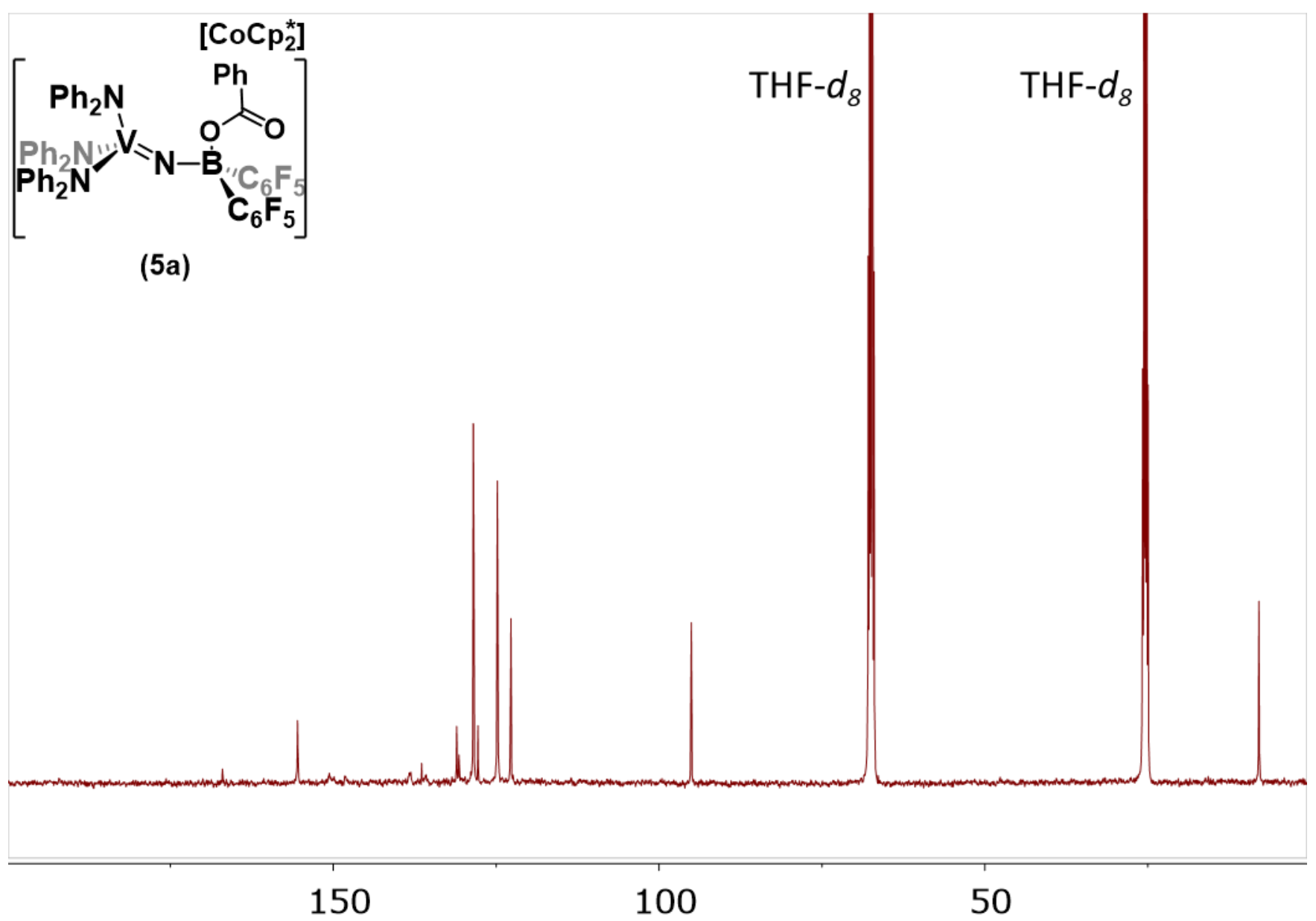

Figure S37. ${ }^{13} \mathrm{C}\left\{{ }^{1} \mathrm{H}\right\}$ NMR spectrum of $\mathbf{5 a}\left(100 \mathrm{MHz}, \mathrm{THF}-d s, 25{ }^{\circ} \mathrm{C}\right)$. 


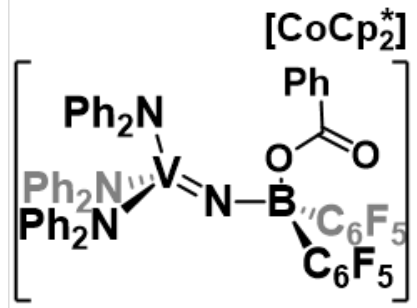

(5a)

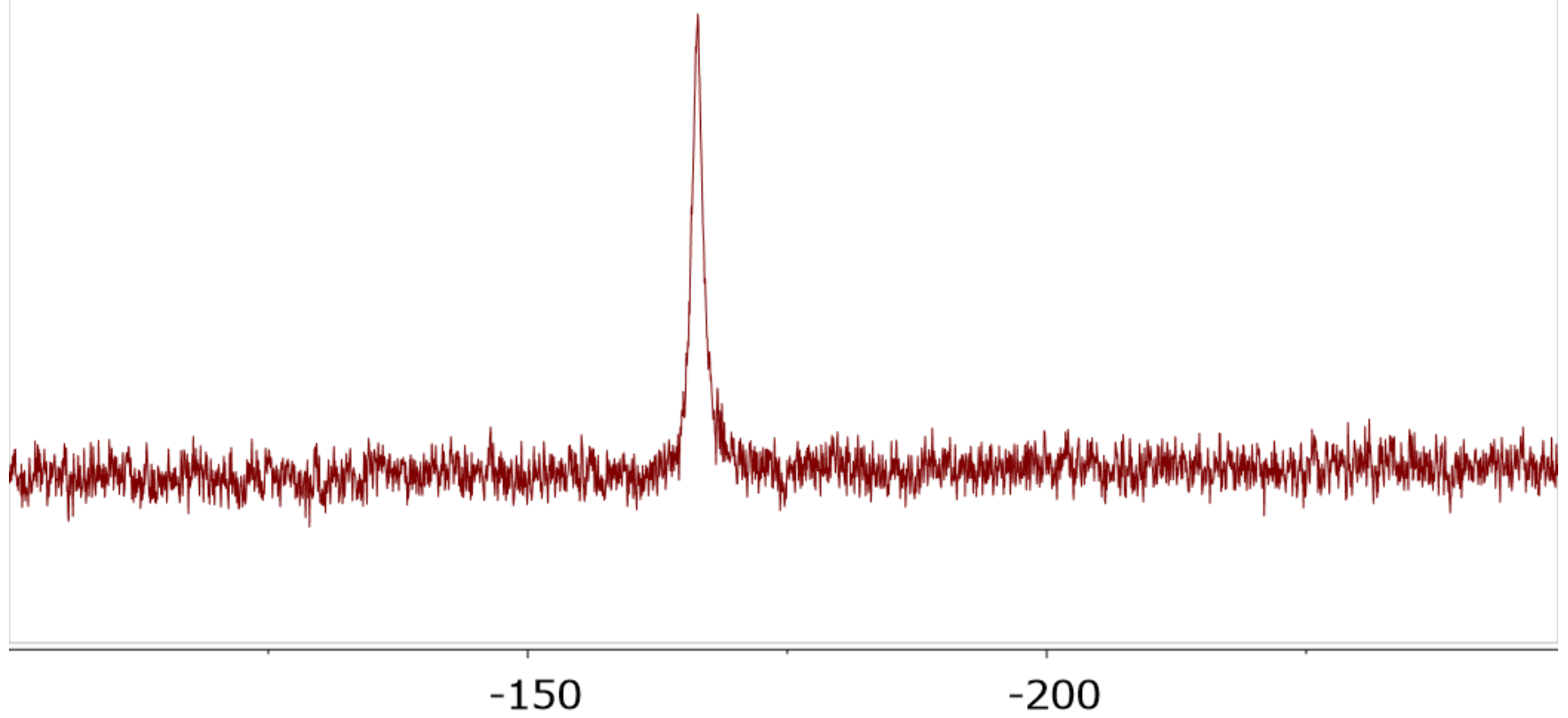

Figure S38. ${ }^{51} \mathrm{~V}$ NMR spectrum of $\mathbf{5 a}\left(105 \mathrm{MHz}, \mathrm{THF}-d 8,25{ }^{\circ} \mathrm{C}\right)$. 


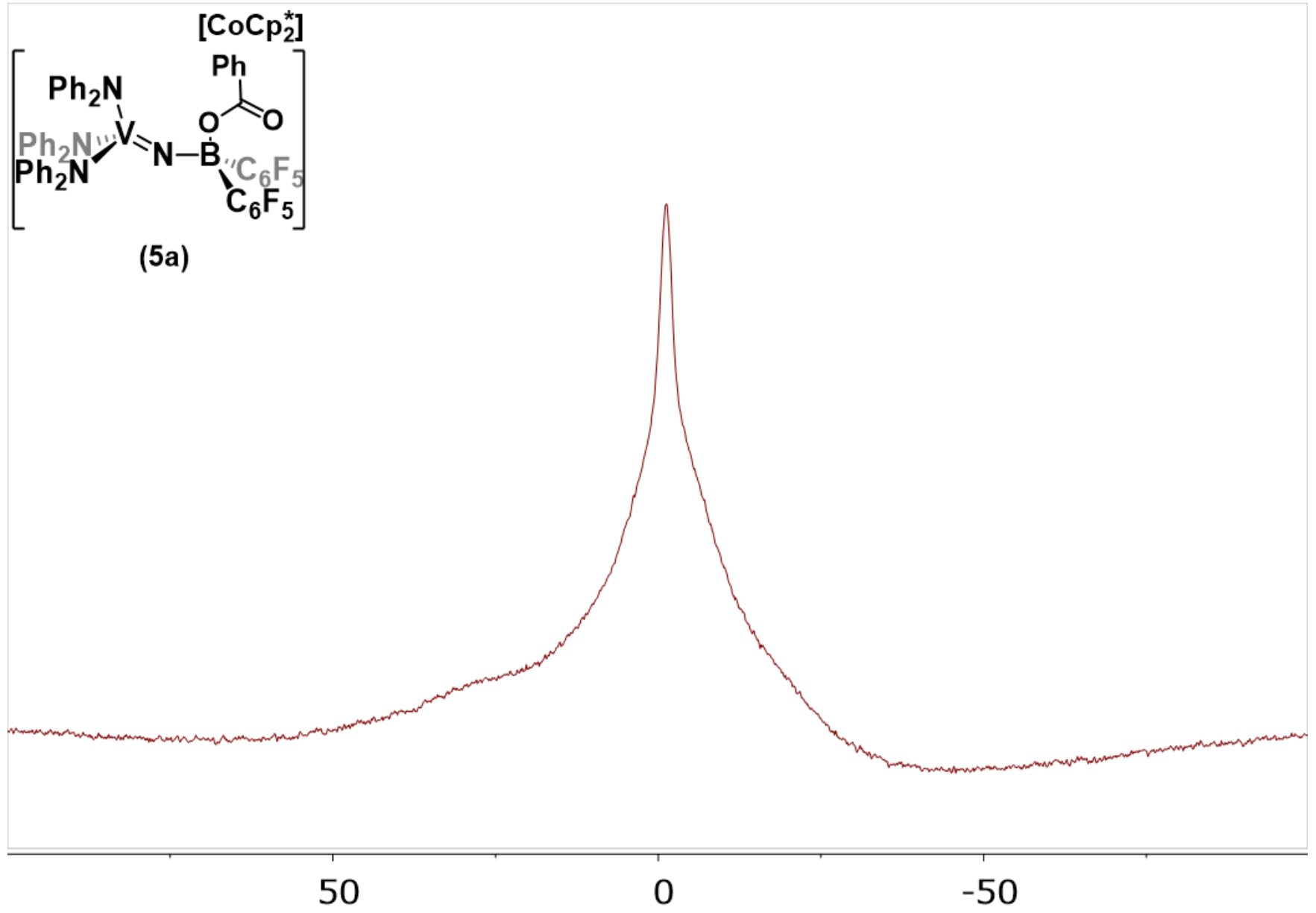

Figure S39. ${ }^{11} \mathrm{~B}\left\{{ }^{1} \mathrm{H}\right\}$ NMR spectrum of 5a $\left(128 \mathrm{MHz}, \mathrm{THF}-d s, 25{ }^{\circ} \mathrm{C}\right)$. 


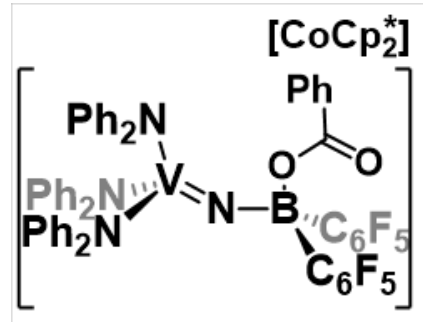

(5a)

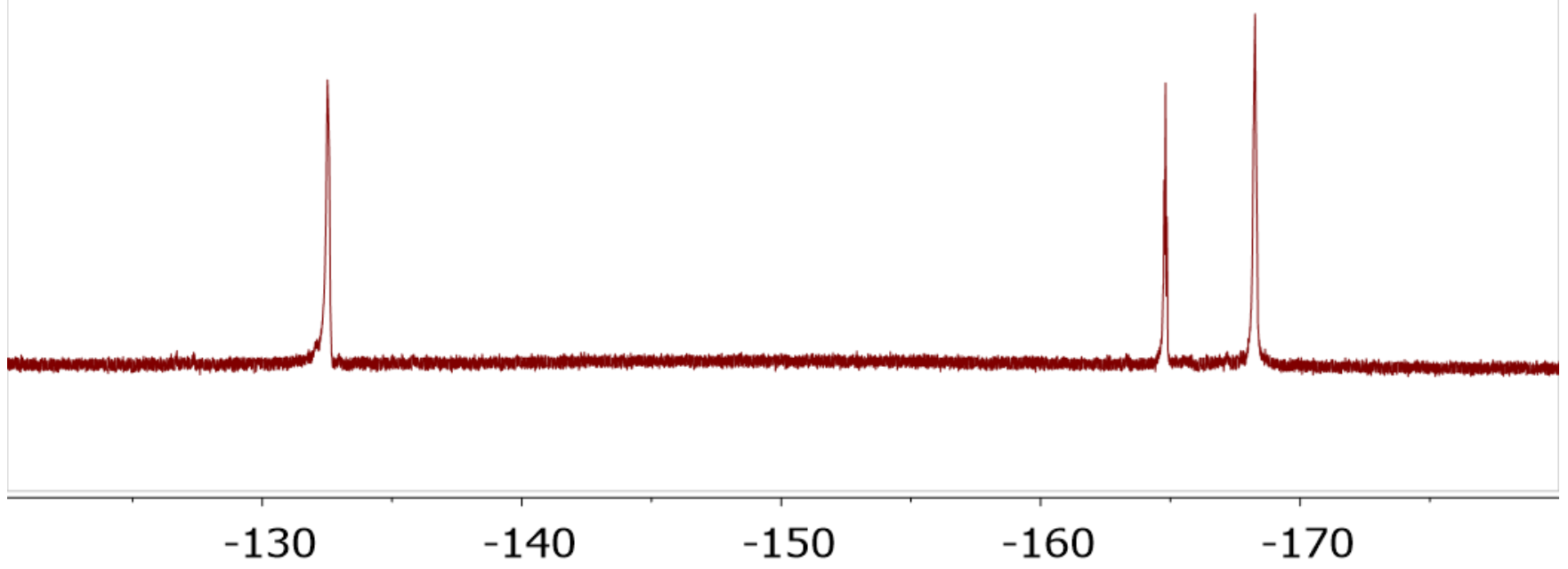

Figure S40. ${ }^{19} \mathrm{~F}\left\{{ }^{1} \mathrm{H}\right\}$ NMR spectrum of $\mathbf{5 a}\left(376 \mathrm{MHz}, \mathrm{THF}-d 8,25{ }^{\circ} \mathrm{C}\right)$. 


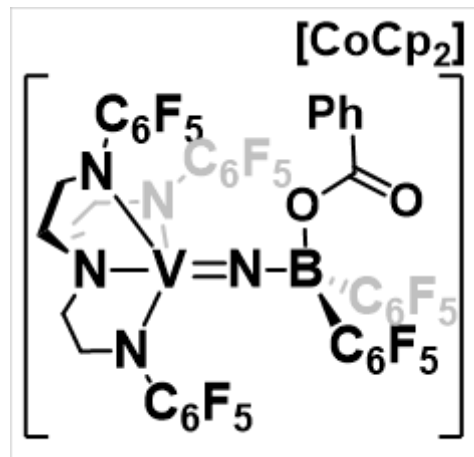

(5b')

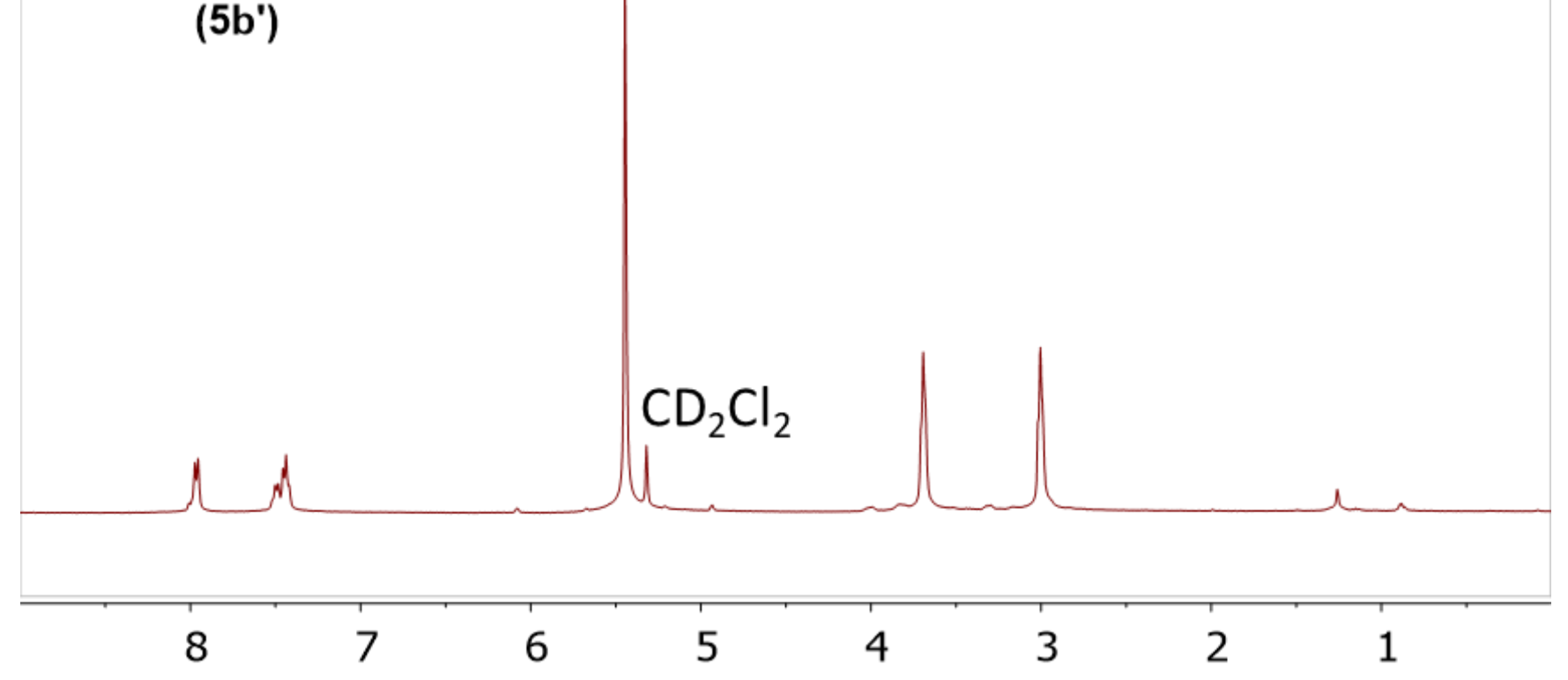

Figure S41. ${ }^{1} \mathrm{H}$ NMR spectrum of $\mathbf{5 b}$ ' (400 MHz, $\left.\mathrm{CD}_{2} \mathrm{Cl}_{2}, 25^{\circ} \mathrm{C}\right)$. 


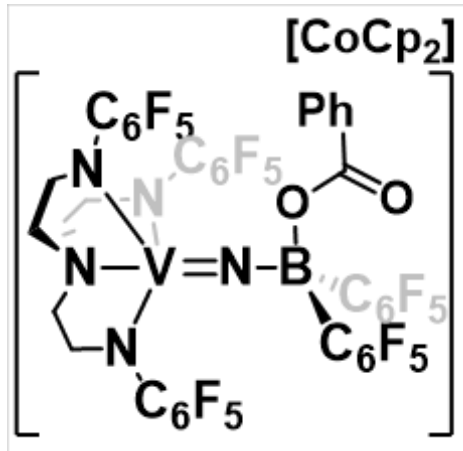

(5b')

\section{$\mathrm{CD}_{2} \mathrm{Cl}_{2}$}

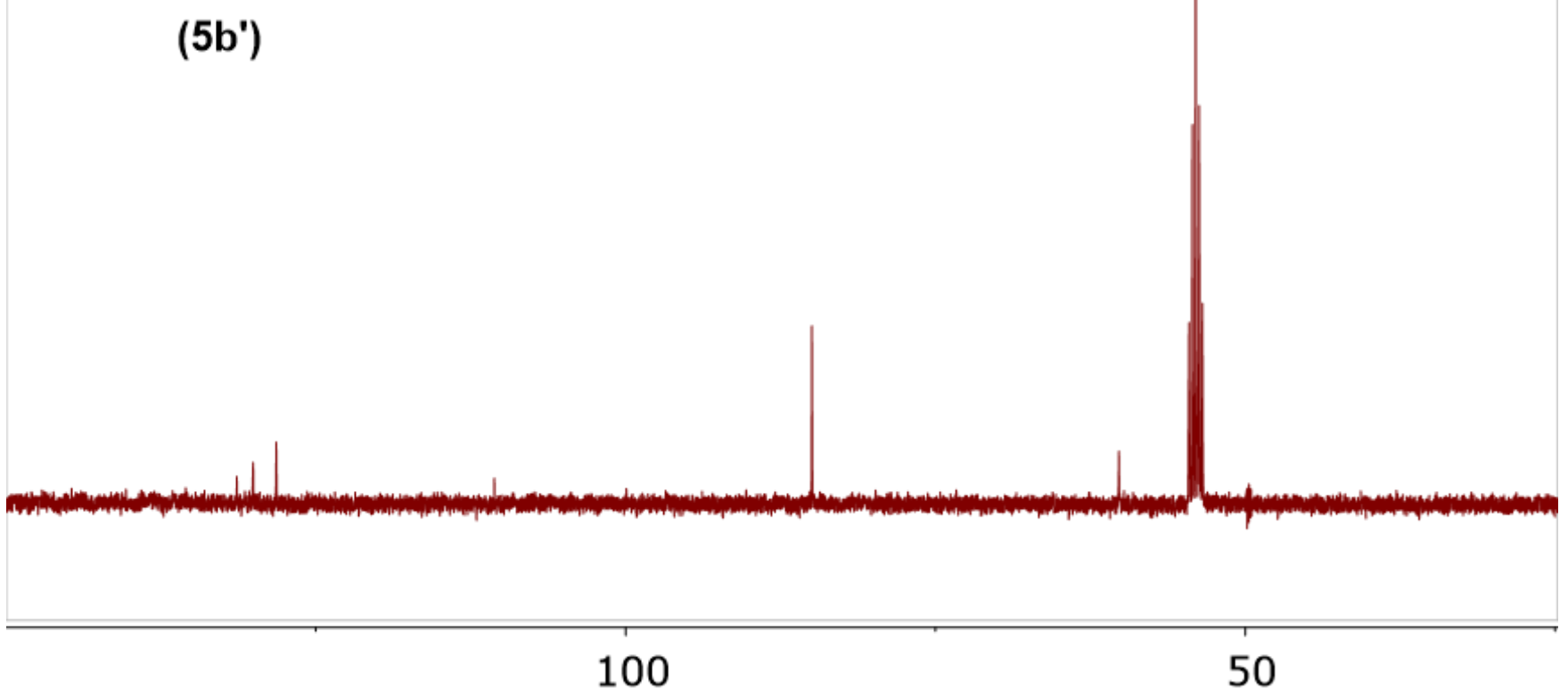

Figure $\mathbf{S 4 2 .}{ }^{13} \mathrm{C}\left\{{ }^{1} \mathrm{H}\right\}$ NMR spectrum of $\mathbf{5} \mathbf{b}^{\prime}\left(100 \mathrm{MHz}, \mathrm{CD}_{2} \mathrm{Cl}_{2}, 25{ }^{\circ} \mathrm{C}\right)$. 


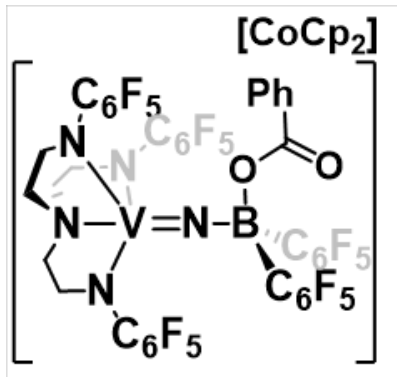

(5b')

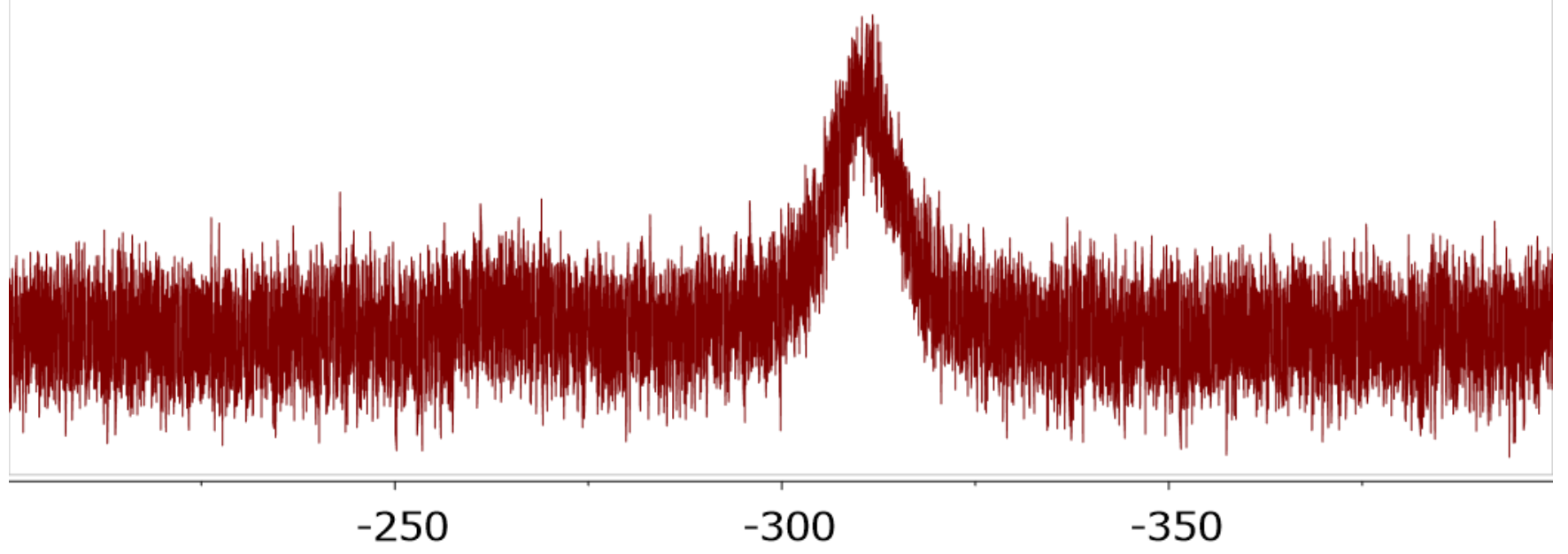

Figure S43. ${ }^{51} \mathrm{~V}$ NMR spectrum of $\mathbf{5} \mathbf{b}^{\prime}\left(105 \mathrm{MHz}, \mathrm{CD}_{2} \mathrm{Cl}_{2}, 25{ }^{\circ} \mathrm{C}\right)$. 


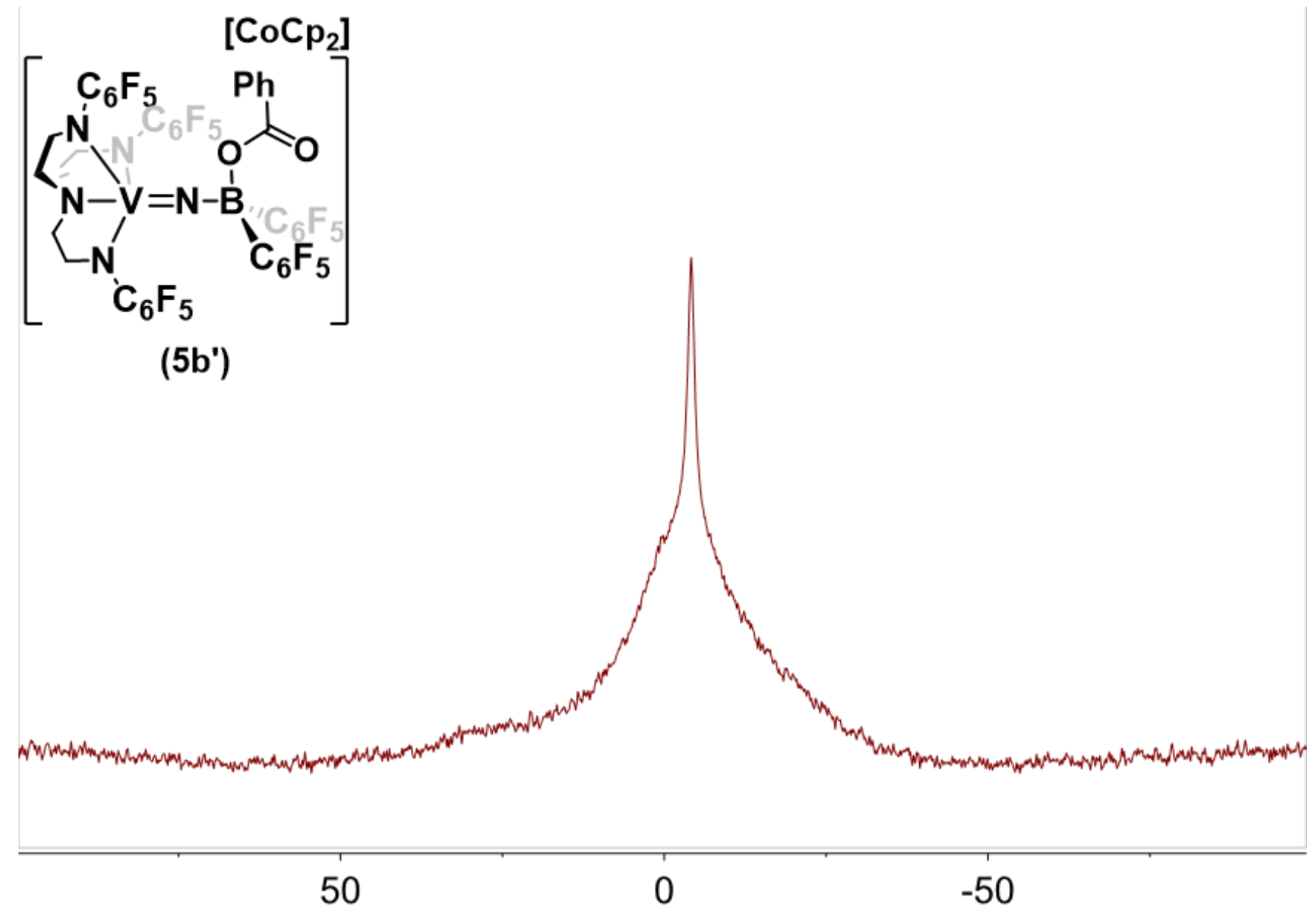

Figure S44. ${ }^{11} \mathrm{~B}\left\{{ }^{1} \mathrm{H}\right\}$ NMR spectrum of $\mathbf{5} \mathbf{b}^{\prime}\left(128 \mathrm{MHz}, \mathrm{CD}_{2} \mathrm{Cl}_{2}, 25{ }^{\circ} \mathrm{C}\right)$. 


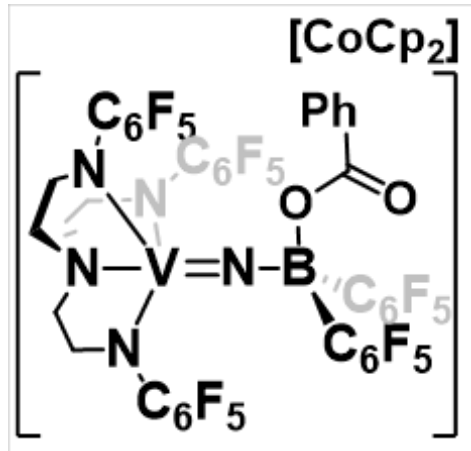

$\left(5 b^{\prime}\right)$

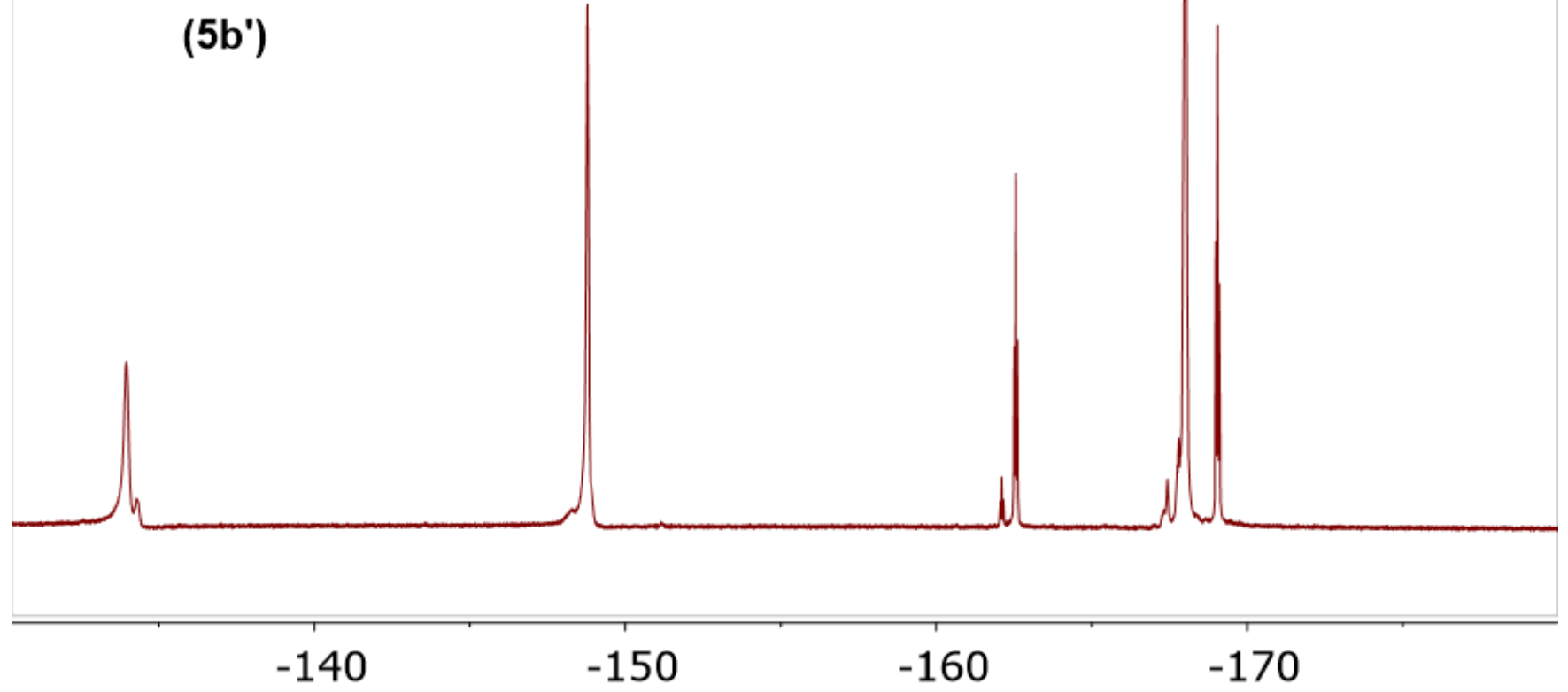

Figure $\mathbf{S 4 5} .{ }^{19} \mathrm{~F}\left\{{ }^{1} \mathrm{H}\right\}$ NMR spectrum of $\mathbf{5} \mathbf{b}^{\prime}\left(376 \mathrm{MHz}, \mathrm{CD}_{2} \mathrm{Cl}_{2}, 25{ }^{\circ} \mathrm{C}\right)$. 
$\mathrm{C}_{6} \mathrm{H}^{\mathrm{N}-\mathrm{H}_{6} \mathrm{H}_{5}} \mathrm{C}_{6} \mathrm{~F}_{5}$

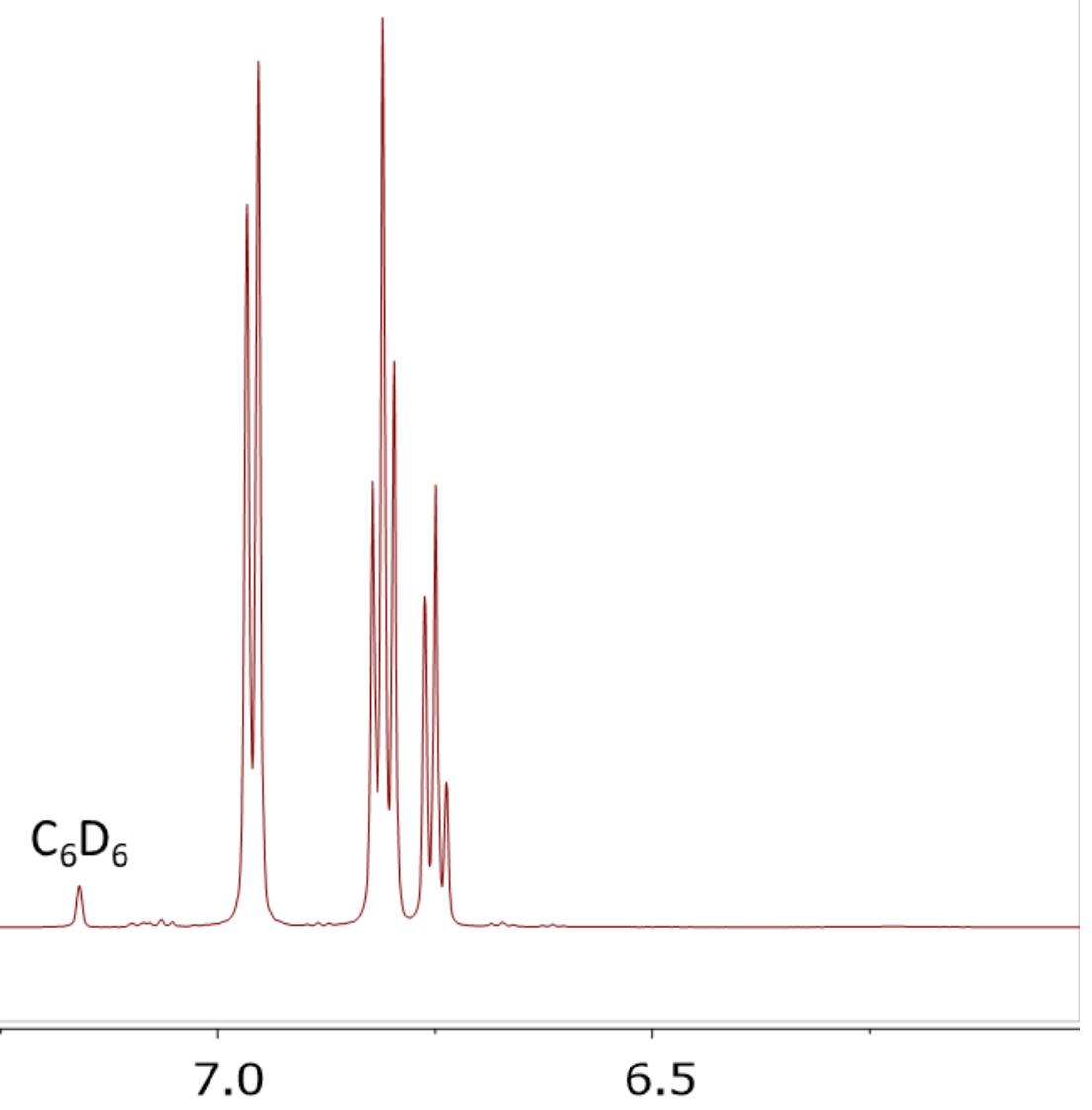

Figure S46. ${ }^{1} \mathrm{H} \mathrm{NMR}$ of $\left(\mathrm{Ph}_{2} \mathrm{~N}\right)_{2} \mathrm{NB}\left(\mathrm{C}_{6} \mathrm{~F}_{5}\right)_{2}\left(600 \mathrm{MHz}, \mathrm{C}_{6} \mathrm{D}_{6}, 25{ }^{\circ} \mathrm{C}\right)$. 


$$
\mathrm{C}_{6} \mathrm{H}^{\mathrm{C}} \mathrm{C}_{6} \mathrm{H}_{5} \mathrm{~F}_{5}
$$$$
\mathrm{C}_{6} \mathrm{D}_{6}
$$

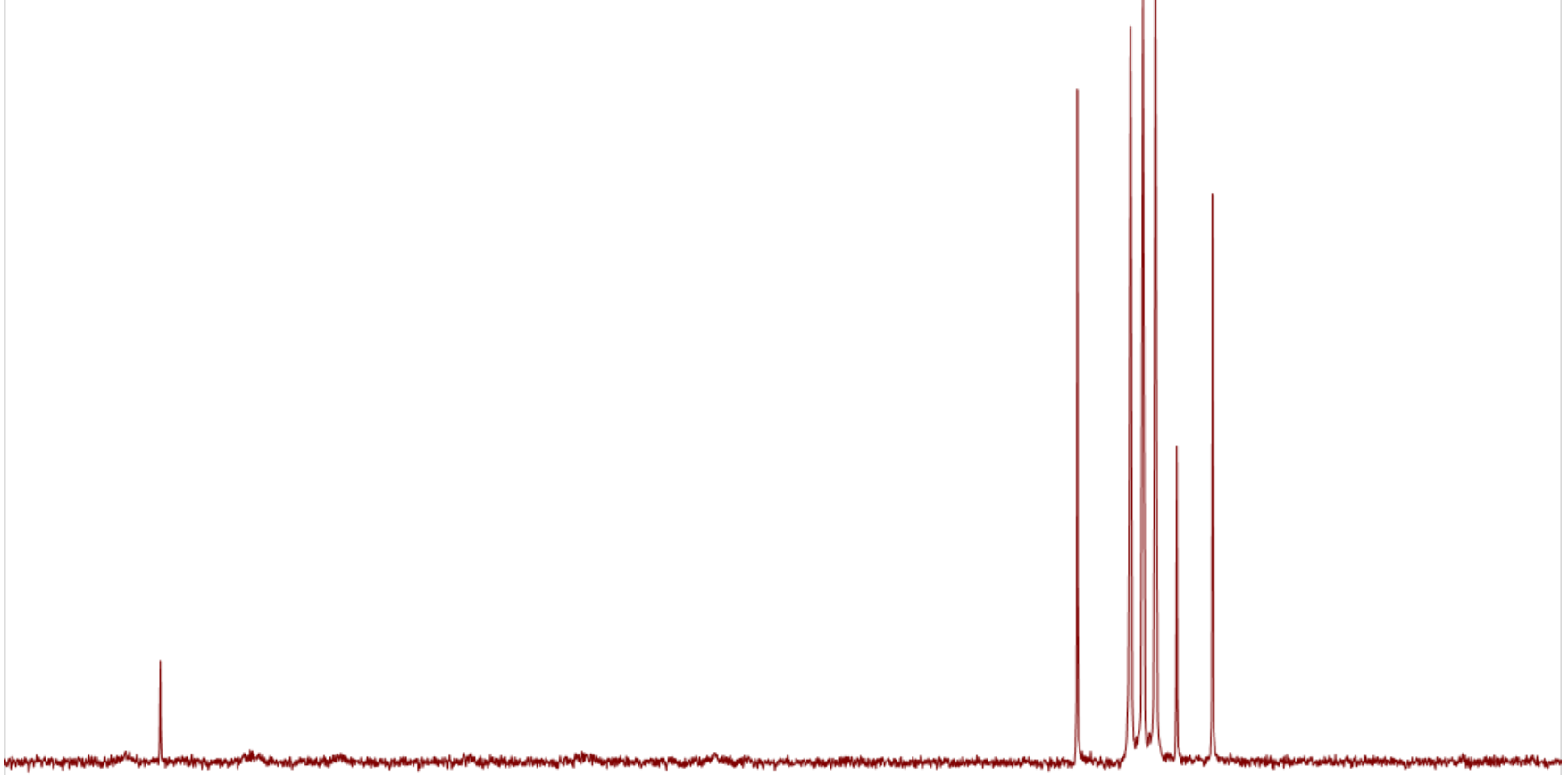

Figure S47. ${ }^{13} \mathrm{C}\left\{{ }^{1} \mathrm{H}\right\}$ NMR spectrum of $\left(\mathrm{Ph}_{2} \mathrm{~N}\right)_{2} \mathrm{NB}\left(\mathrm{C}_{6} \mathrm{~F}_{5}\right)_{2}\left(100 \mathrm{MHz}, \mathrm{C}_{6} \mathrm{D}_{6}, 25{ }^{\circ} \mathrm{C}\right)$. 
$\mathrm{C}_{6} \mathrm{H}_{5}^{\mathrm{N}-\mathrm{C}_{6} \mathrm{H}_{5}} \stackrel{\mathrm{C}_{6} \mathrm{~F}_{5}}{\mathrm{C}_{6} \mathrm{~F}_{5}}$

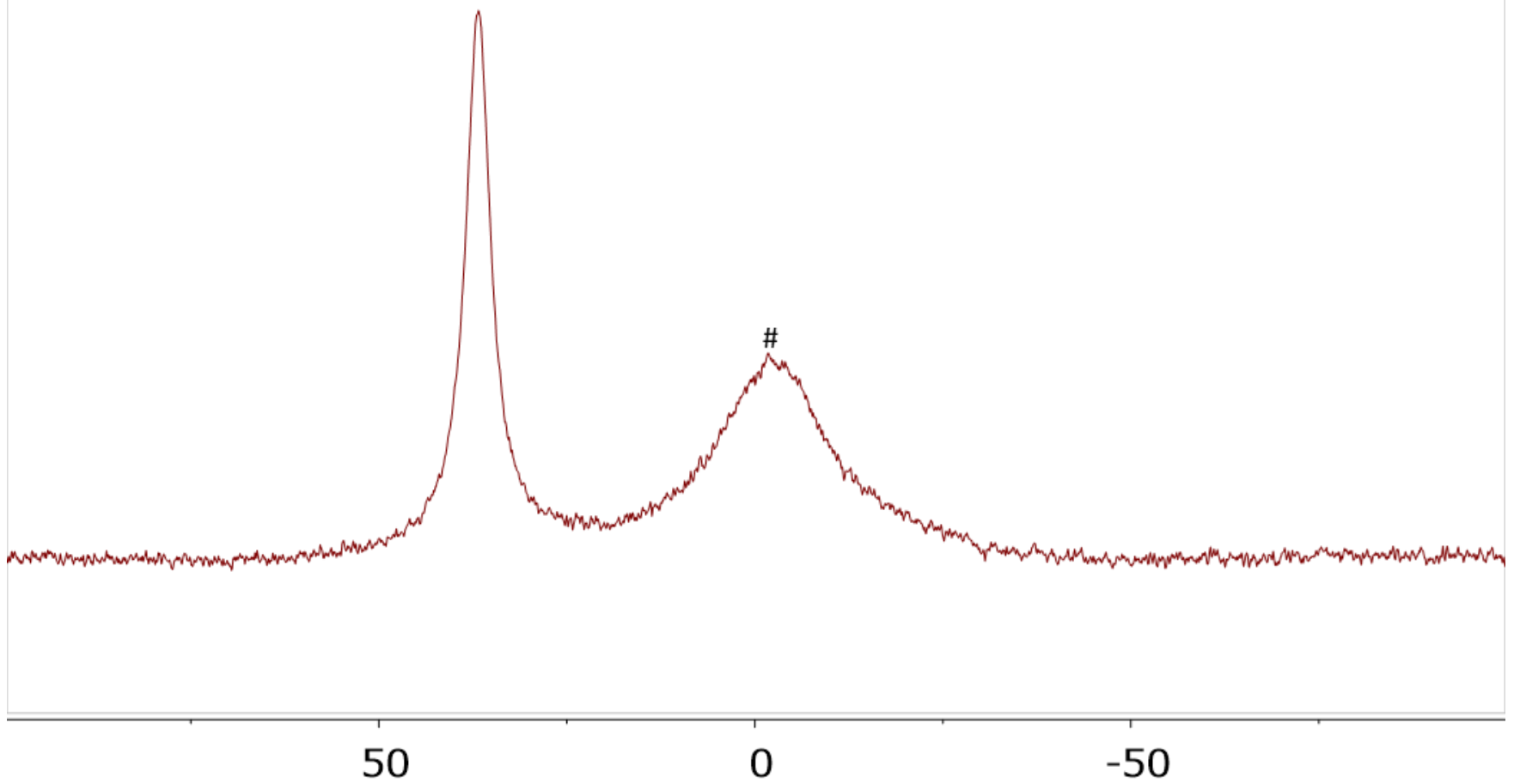

Figure S48. ${ }^{11} \mathrm{~B}\left\{{ }^{1} \mathrm{H}\right\}$ NMR spectrum of $\left(\mathrm{Ph}_{2} \mathrm{~N}\right)_{2} \mathrm{NB}\left(\mathrm{C}_{6} \mathrm{~F}_{5}\right)_{2}\left(128 \mathrm{MHz}, \mathrm{C}_{6} \mathrm{D}_{6}, 25{ }^{\circ} \mathrm{C}\right)$. (\# = borosilicate resonance from NMR tube) 
$\mathrm{C}_{6} \mathrm{H}_{5}^{\mathrm{C}-\mathrm{C}_{6} \mathrm{H}_{5}} \stackrel{\mathrm{C}_{6} \mathrm{~F}_{5}}{\mathrm{C}_{5}}$
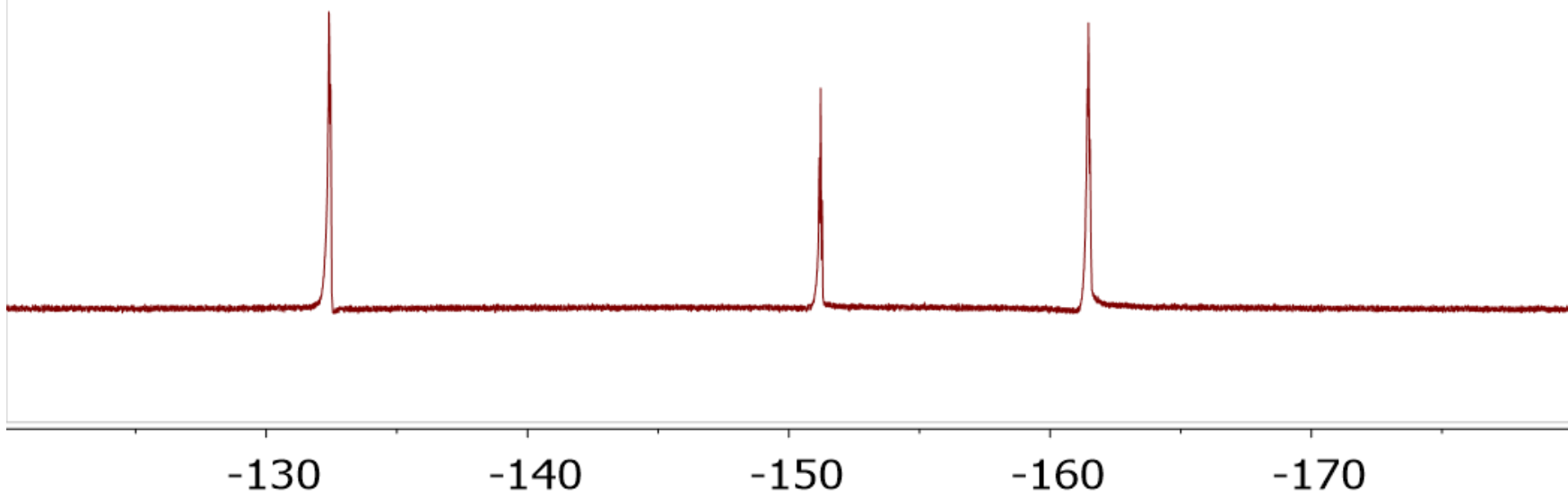

Figure S49. ${ }^{19} \mathrm{~F}\left\{{ }^{1} \mathrm{H}\right\}$ NMR spectrum of $\left(\mathrm{Ph}_{2} \mathrm{~N}\right)_{2} \mathrm{NB}\left(\mathrm{C}_{6} \mathrm{~F}_{5}\right)_{2}\left(376 \mathrm{MHz}, \mathrm{C}_{6} \mathrm{D}_{6}, 25{ }^{\circ} \mathrm{C}\right)$. 


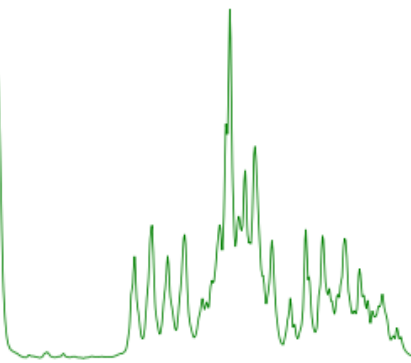

\section{r.t. $5 \mathrm{~min}$}

\begin{tabular}{|c|c|c|c|c|c|c|c|c|c|c|c|c|c|c|c|c|c|c|}
\hline 1 & 1 & 1 & 1 & 1 & 1 & 1 & 1 & 1 & 1 & 1 & 1 & 1 & 1 & 1 & 1 & 1 & 1 & 1 \\
\hline 7.9 & 7.8 & 7.7 & 7.6 & 7.5 & 7.4 & 7.3 & 7.2 & 7.1 & $\begin{array}{c}7.0 \\
\text { f1 (ppm) }\end{array}$ & 6.9 & 6.8 & 6.7 & 6.6 & 6.5 & 6.4 & 6.3 & 6.2 & 6.1 \\
\hline
\end{tabular}

Figure S50. Stacked ${ }^{1} \mathrm{H}$ NMR spectra monitoring the decomposition of $\mathbf{1 a}\left(400 \mathrm{MHz}, \mathrm{C}_{6} \mathrm{D}_{6}, 25{ }^{\circ} \mathrm{C}\right)$. 


\section{r.t. $16 \mathrm{~h}$}

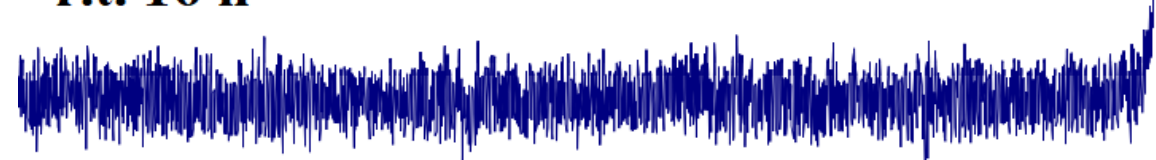

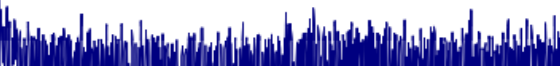
Nin

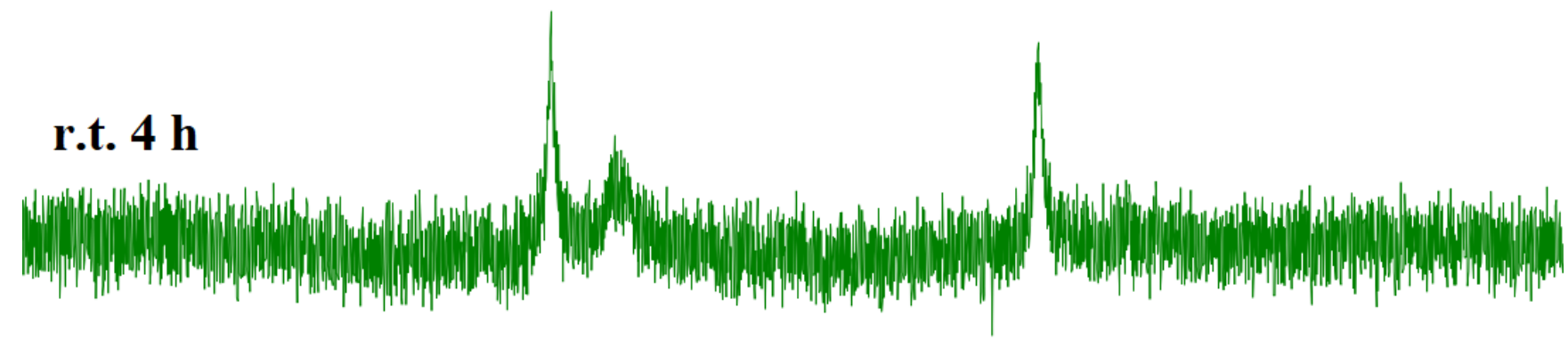

\section{r.t. $5 \mathrm{~min}$}

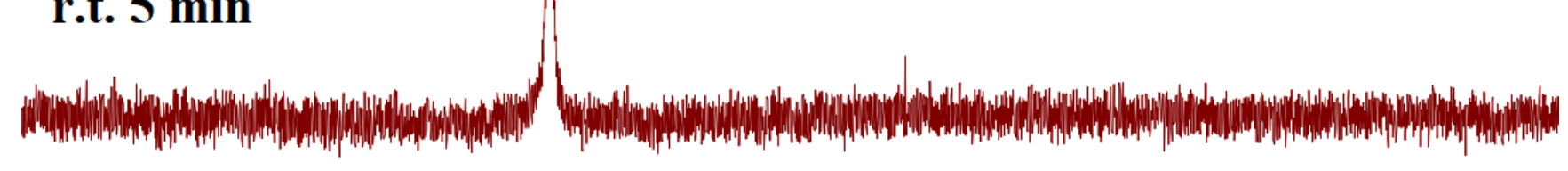

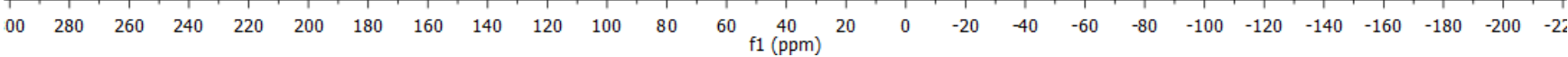

Figure S51. Stacked ${ }^{51} \mathrm{~V}$ NMR spectra monitoring the decomposition of $\mathbf{1 a}\left(105 \mathrm{MHz}, \mathrm{C}_{6} \mathrm{D}_{6}, 25{ }^{\circ} \mathrm{C}\right)$. 

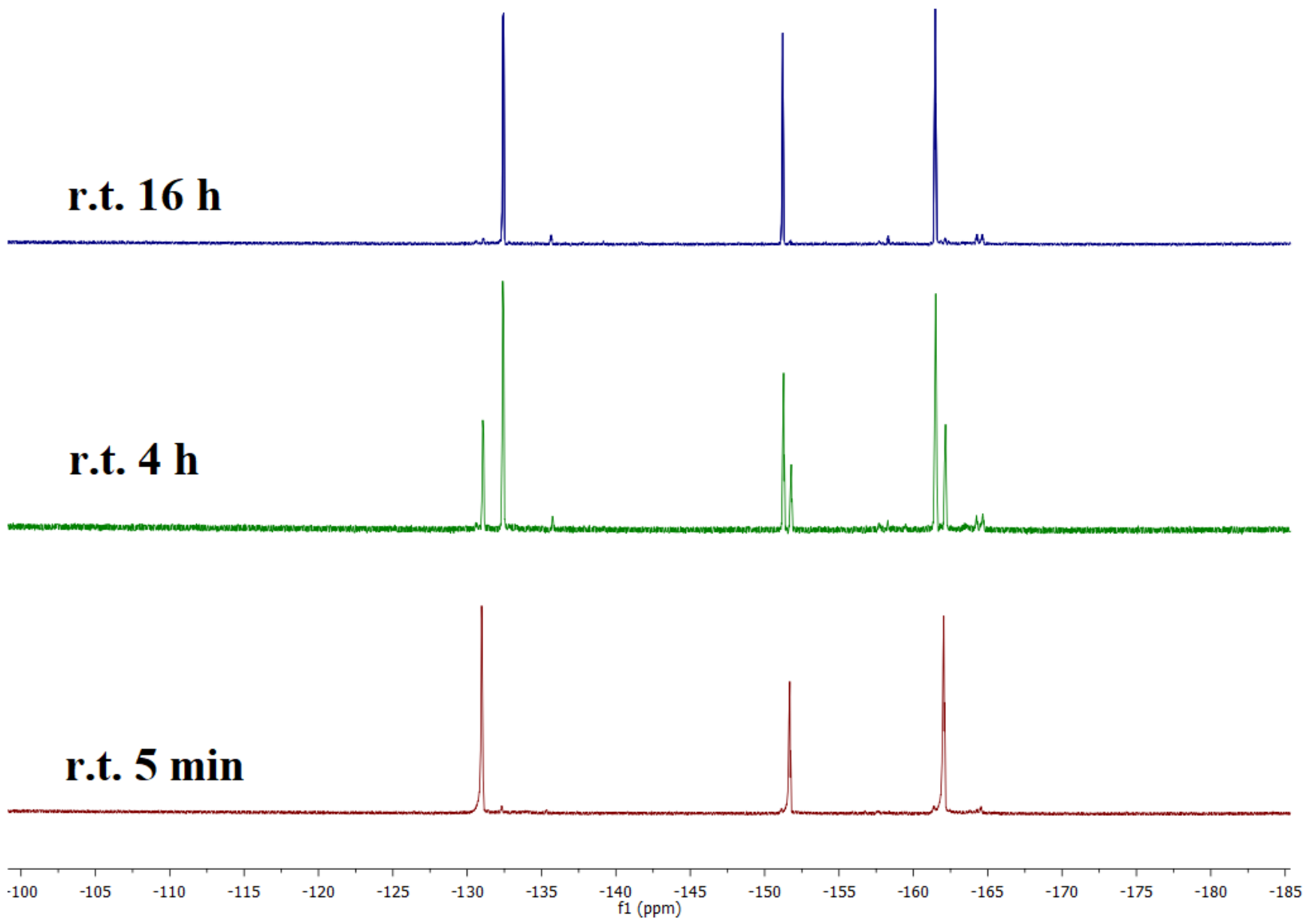

Figure S52. Stacked ${ }^{19} \mathrm{~F}\left\{{ }^{1} \mathrm{H}\right\}$ NMR spectra monitoring the decomposition of $\mathbf{1 a}\left(376 \mathrm{MHz}, \mathrm{C}_{6} \mathrm{D}_{6}, 25{ }^{\circ} \mathrm{C}\right)$. 


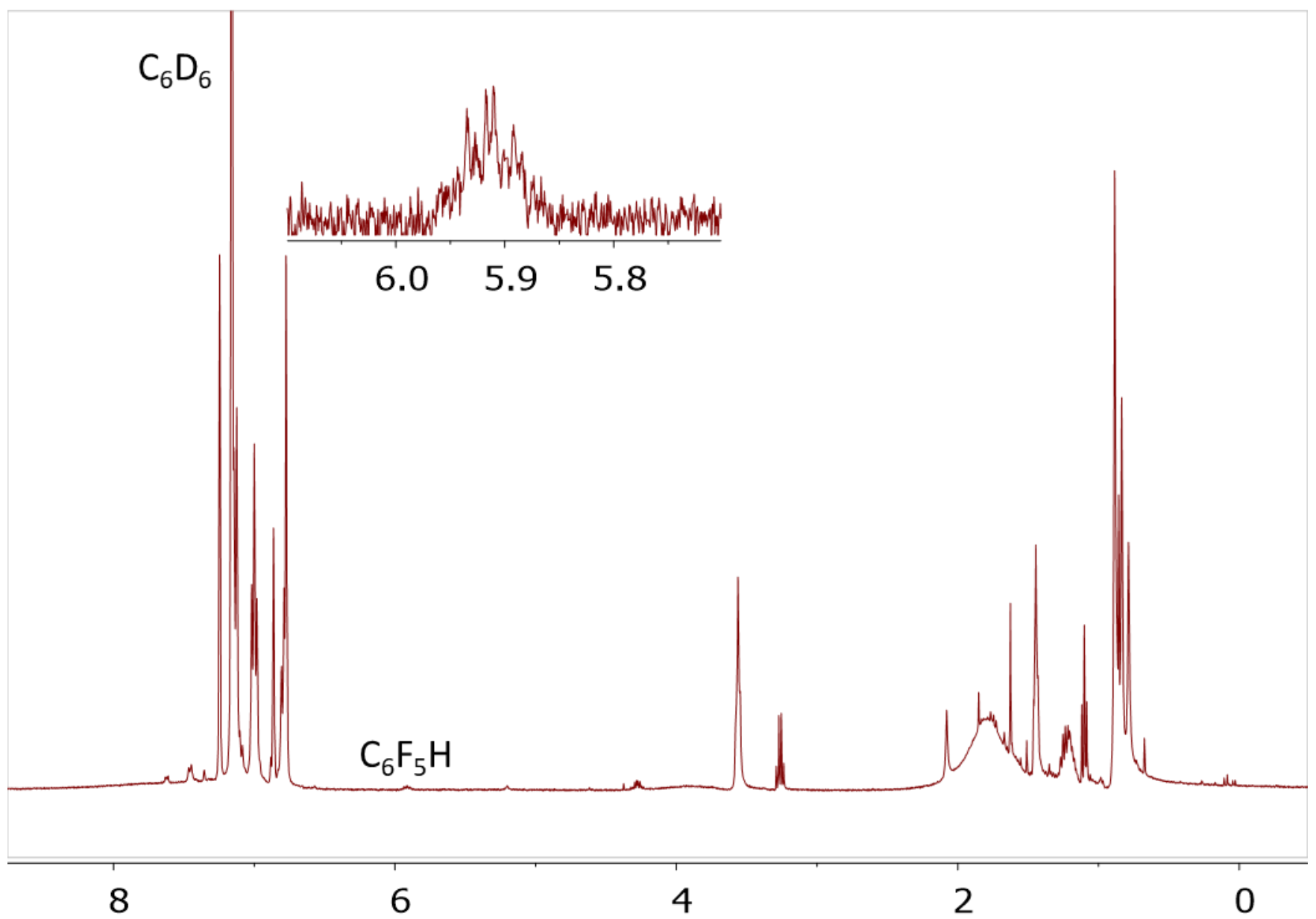

Figure S53. Crude ${ }^{1} \mathrm{H}$ NMR spectrum resulting from the decomposition of $\mathbf{3 a}$ to $\mathbf{4 a}$ and some byproducts, including trace $\mathrm{C}_{6} \mathrm{~F}_{5} \mathrm{H}$, after standing at room temperature for $24 \mathrm{~h}\left(400 \mathrm{MHz}, \mathrm{C}_{6} \mathrm{D}_{6} / \mathrm{C}_{6} \mathrm{D}_{5} \mathrm{Br}(1: 1), 25^{\circ} \mathrm{C}\right)$. 


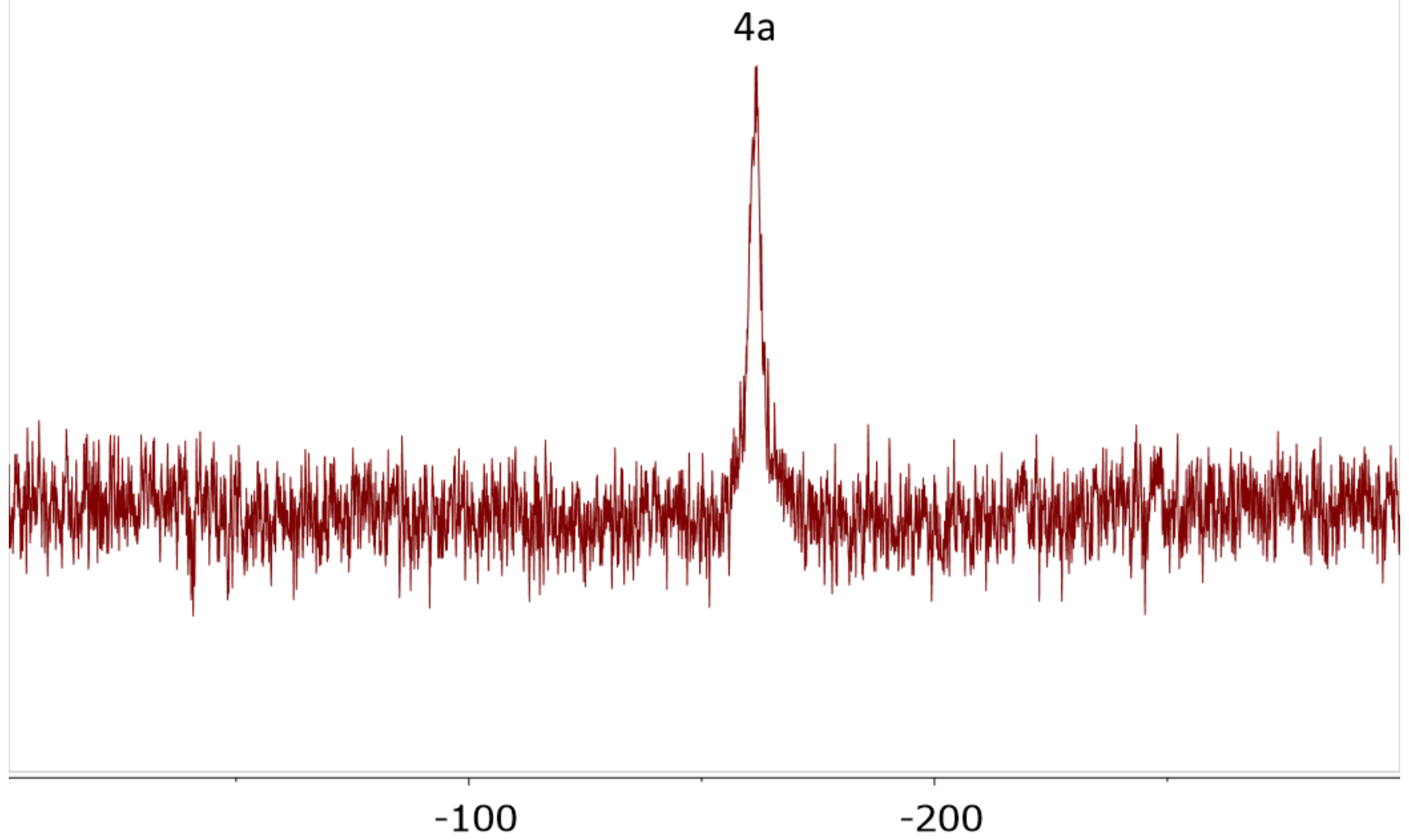

Figure S54. Crude ${ }^{51} \mathrm{~V}$ NMR spectrum resulting from the decomposition of 3a to 4a after standing at room temperature for $24 \mathrm{~h}\left(105 \mathrm{MHz}, \mathrm{C}_{6} \mathrm{D}_{6} / \mathrm{C}_{6} \mathrm{D} 5 \mathrm{Br}(1: 1), 25^{\circ} \mathrm{C}\right)$. 


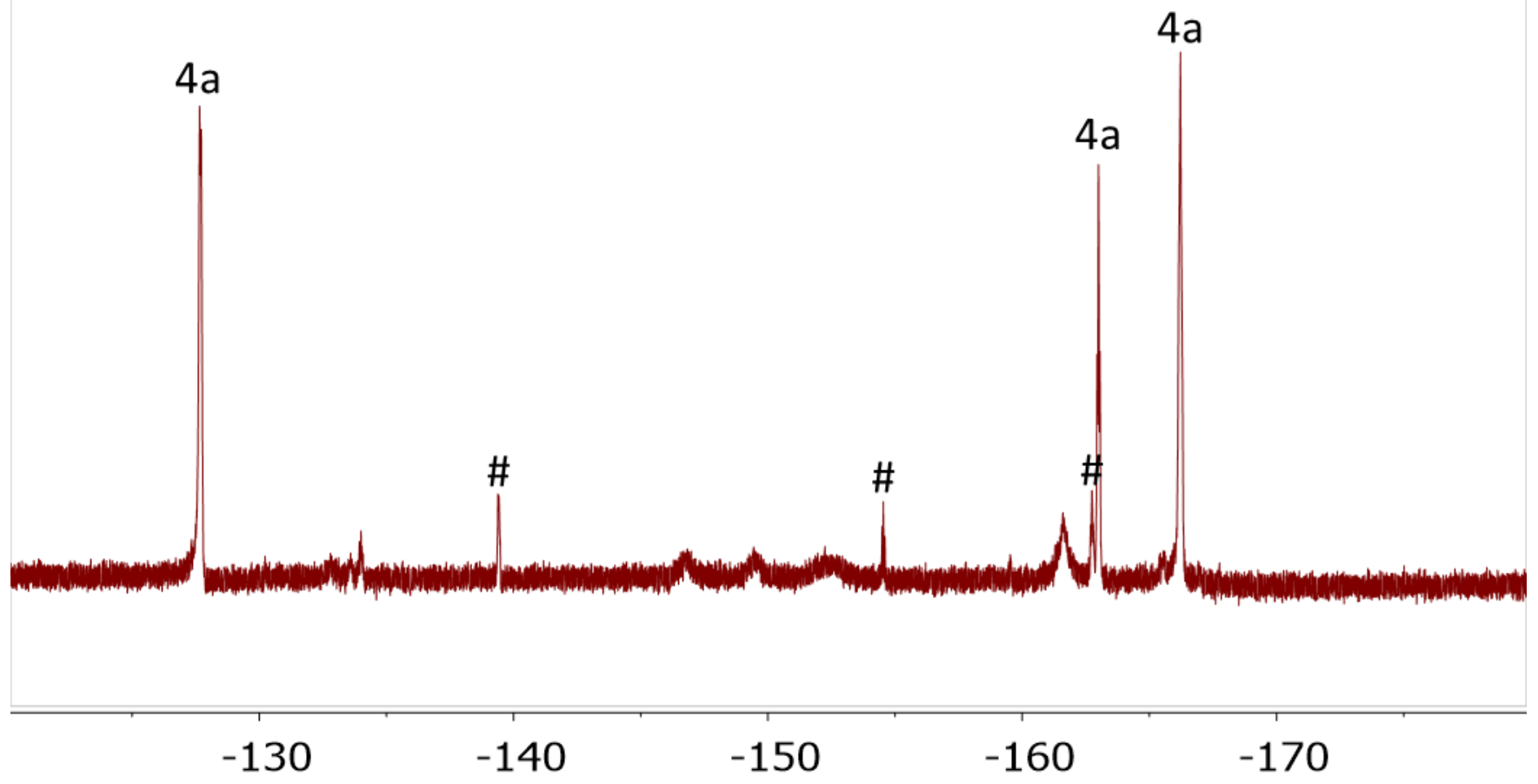

Figure S55. Crude ${ }^{19} \mathrm{~F}\left\{{ }^{1} \mathrm{H}\right\}$ NMR spectrum from the decomposition of 3a to $\mathbf{4 a}$ as the main product (>85\%) after standing at room temperature for $24 \mathrm{~h}$. Trace $\mathrm{C}_{6} \mathrm{~F}_{5} \mathrm{H}$ (\#) is also observed among other unknown byproducts $\left(376 \mathrm{MHz}, \mathrm{C}_{6} \mathrm{D}_{6} / \mathrm{C}_{6} \mathrm{D}_{5} \mathrm{Br}(1: 1), 25^{\circ} \mathrm{C}\right)$. 


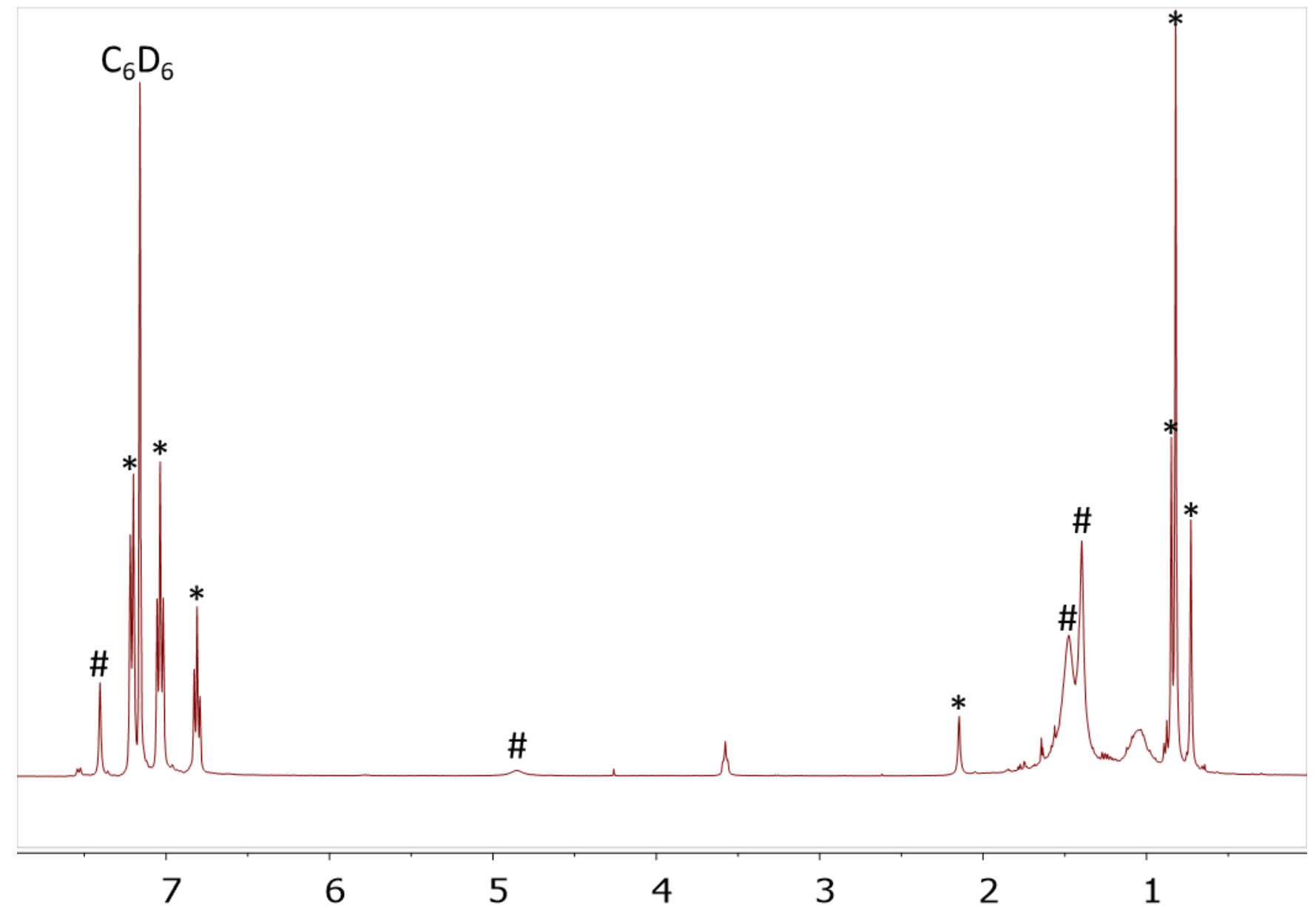

Figure S56. Crude ${ }^{1} \mathrm{H}$ NMR spectrum resulting from the reaction of $\mathbf{3 a}$ with 2,4,6- ${ }^{t} \mathrm{Bu}_{3} \mathrm{C}_{6} \mathrm{H}_{2} \mathrm{O} \bullet$ to produce $4 \mathbf{a}\left(^{*}\right)$ and $2,4,6-{ }^{t} \mathrm{Bu}_{3} \mathrm{PhOH}(\#)$ in a $1: 1$ ratio $\left(400 \mathrm{MHz}, \mathrm{C}_{6} \mathrm{D}_{6}, 25{ }^{\circ} \mathrm{C}\right) .{ }^{51} \mathrm{~V},{ }^{11} \mathrm{~B}$, and ${ }^{19} \mathrm{~F}$ NMR spectra were the same as those of pure, isolated $4 \mathbf{a}$. 

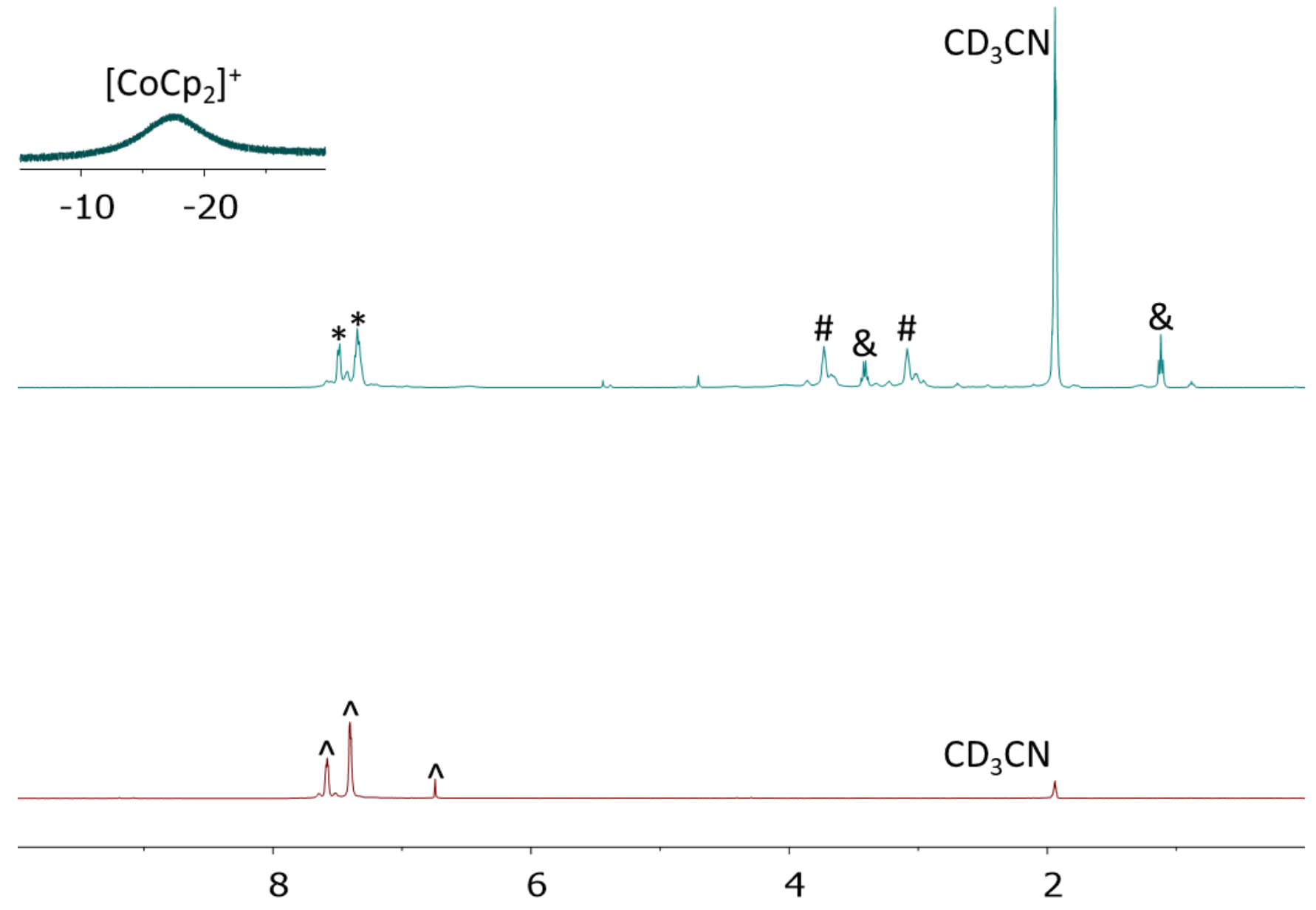

Figure S57. (bottom) ${ }^{1} \mathrm{H}$ NMR spectrum of $\mathrm{Ph}_{3} \mathrm{SnH}\left({ }^{\wedge}\right)$. (top) Crude ${ }^{1} \mathrm{H}$ NMR spectrum following the reaction of $\mathbf{3} \mathbf{b}^{\prime}$ with $\mathrm{Ph}_{3} \mathrm{SnH}$ showing complete consumption of $\mathrm{Ph}_{3} \mathrm{SnH}$, aromatic resonances for $\mathrm{Ph}_{3} \mathrm{Sn}-$ $\mathrm{SnPh}_{3}(*)$, resonances for a tren-containing species (\#), and a upfield $\left[\mathrm{CoCp}_{2}\right]^{+}$resonance $(400 \mathrm{MHz}$, $\left.\mathrm{CD}_{3} \mathrm{CN}, 25^{\circ} \mathrm{C}\right)$. 


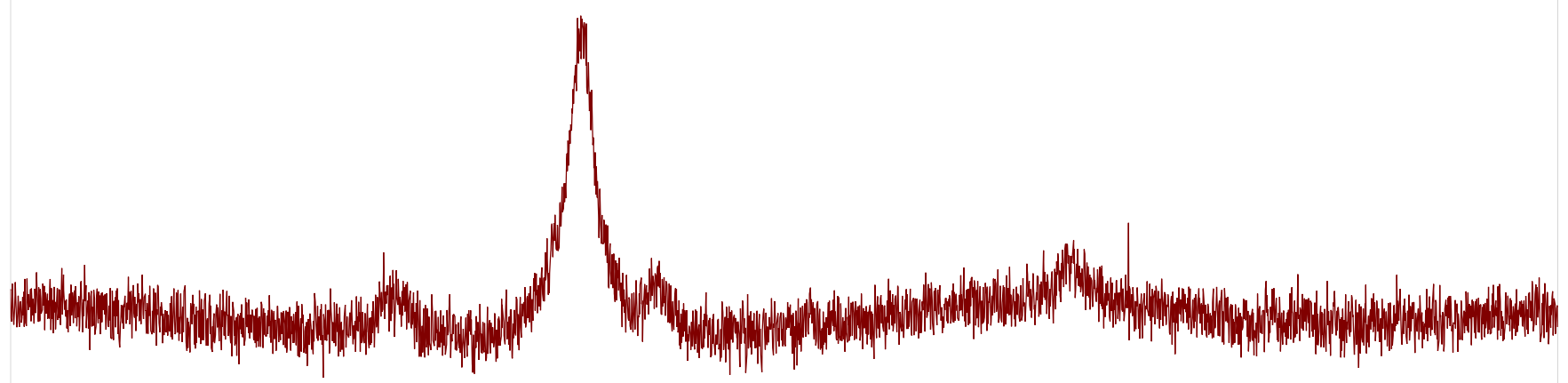

\section{$-300-400$}

Figure S58. Crude ${ }^{51} \mathrm{~V}$ NMR spectrum from the reaction between $\mathbf{3} \mathbf{b}^{\prime}$ with $\mathrm{Ph} 3 \mathrm{SnH}$ showing a major vanadium species at $-310.9 \mathrm{ppm}$ after complete consumption of $\mathrm{Ph}_{3} \mathrm{SnH}\left(105 \mathrm{MHz}, \mathrm{CD}_{3} \mathrm{CN}, 25{ }^{\circ} \mathrm{C}\right)$. 


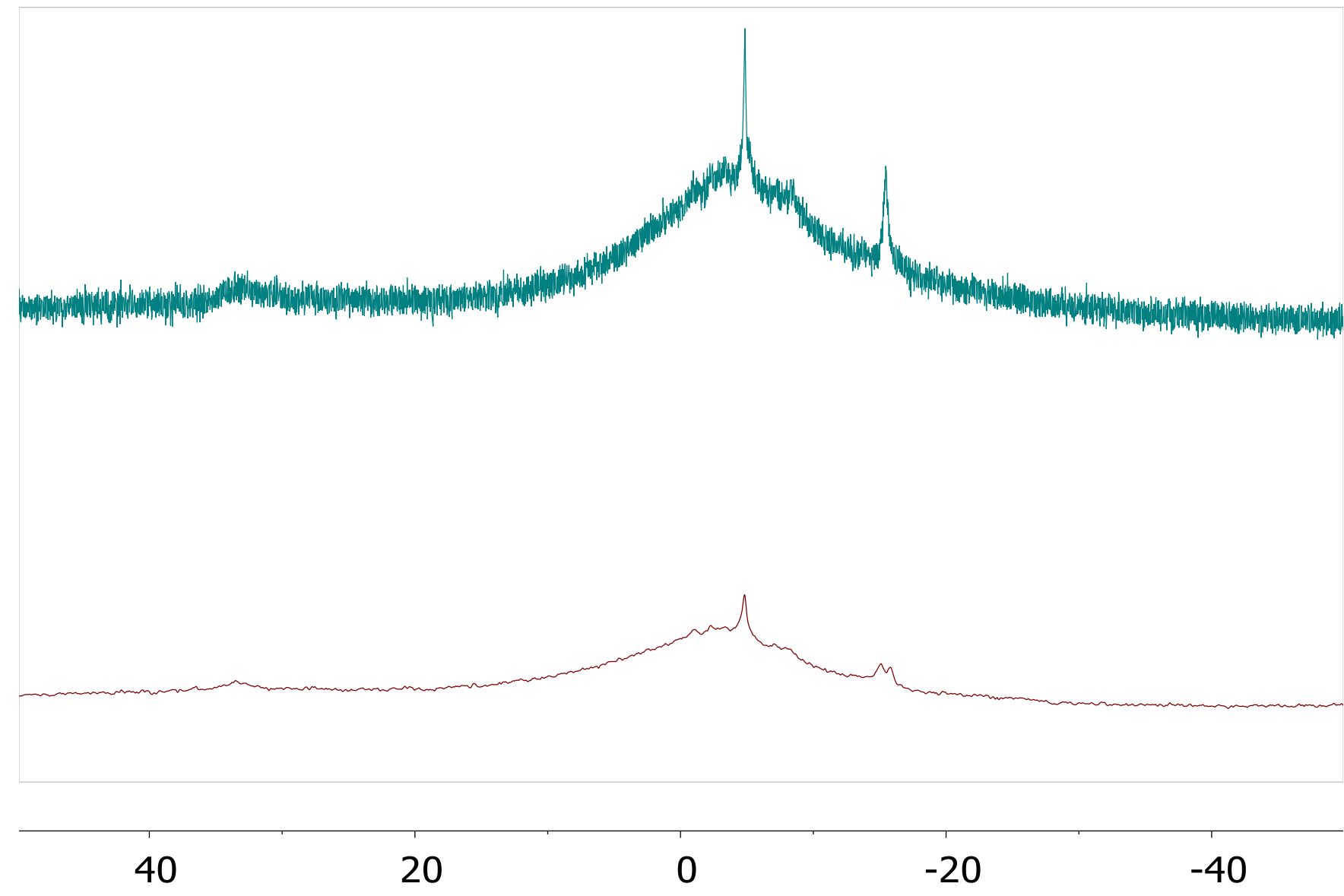

Figure S59. (bottom) Crude ${ }^{11} \mathrm{~B}$ NMR spectrum from the reaction between $\mathbf{3} \mathbf{b}^{\prime}$ and $\mathrm{Ph}_{3} \mathrm{SnH}$ showing a doublet at -15.4 ppm after complete consumption of $\mathrm{Ph} 3 \mathrm{SnH}$. (top) ${ }^{11} \mathrm{~B}\left\{{ }^{1} \mathrm{H}\right\} \mathrm{NMR}$ spectrum of the same reaction showing the doublet resonance collapsing into a singlet, indicative of a $\mathrm{B}-\mathrm{H}$ bond $(128 \mathrm{MHz}$, $\left.\mathrm{CD}_{3} \mathrm{CN}, 25^{\circ} \mathrm{C}\right)$. 


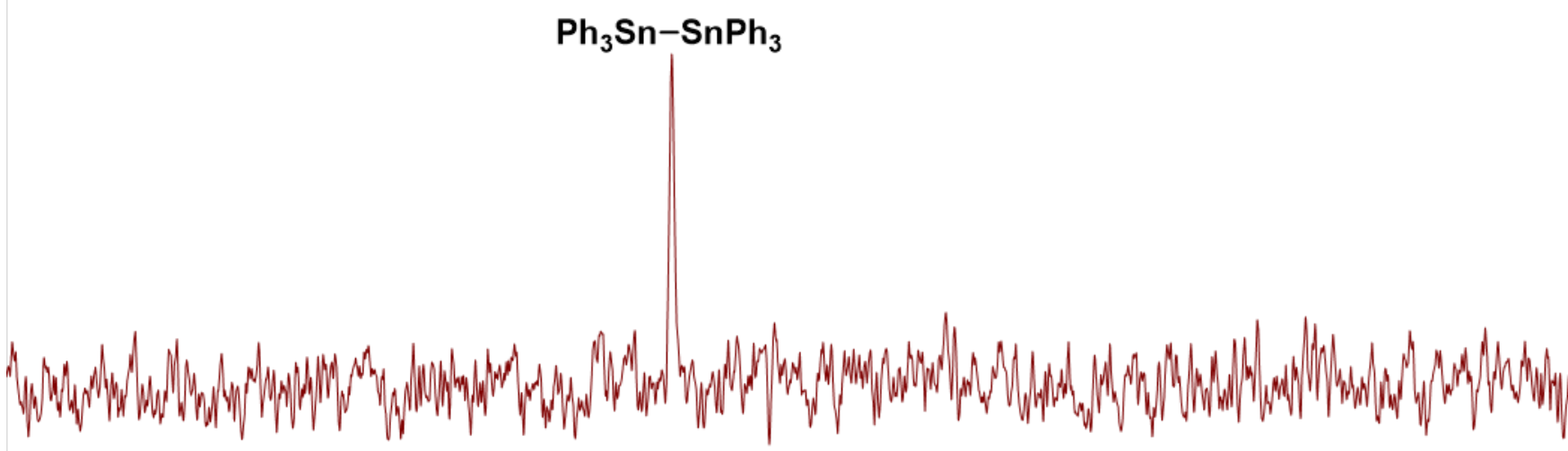

$-120 \quad-140 \quad-160 \quad-180$

Figure S60. Crude ${ }^{119} \mathrm{Sn}$ NMR spectrum from the reaction between $\mathbf{3} \mathbf{b}^{\prime}$ with $\mathrm{Ph}_{3} \mathrm{SnH}$ revealing a lone singlet at $-142.4 \mathrm{ppm}$ that is assigned to $\mathrm{Ph}_{3} \mathrm{Sn}-\mathrm{SnPh}_{3}$ and further corroborated by XRD studies (see manuscript. (149 $\mathrm{MHz}, \mathrm{CD}_{3} \mathrm{CN}, 25^{\circ} \mathrm{C}$ ). 


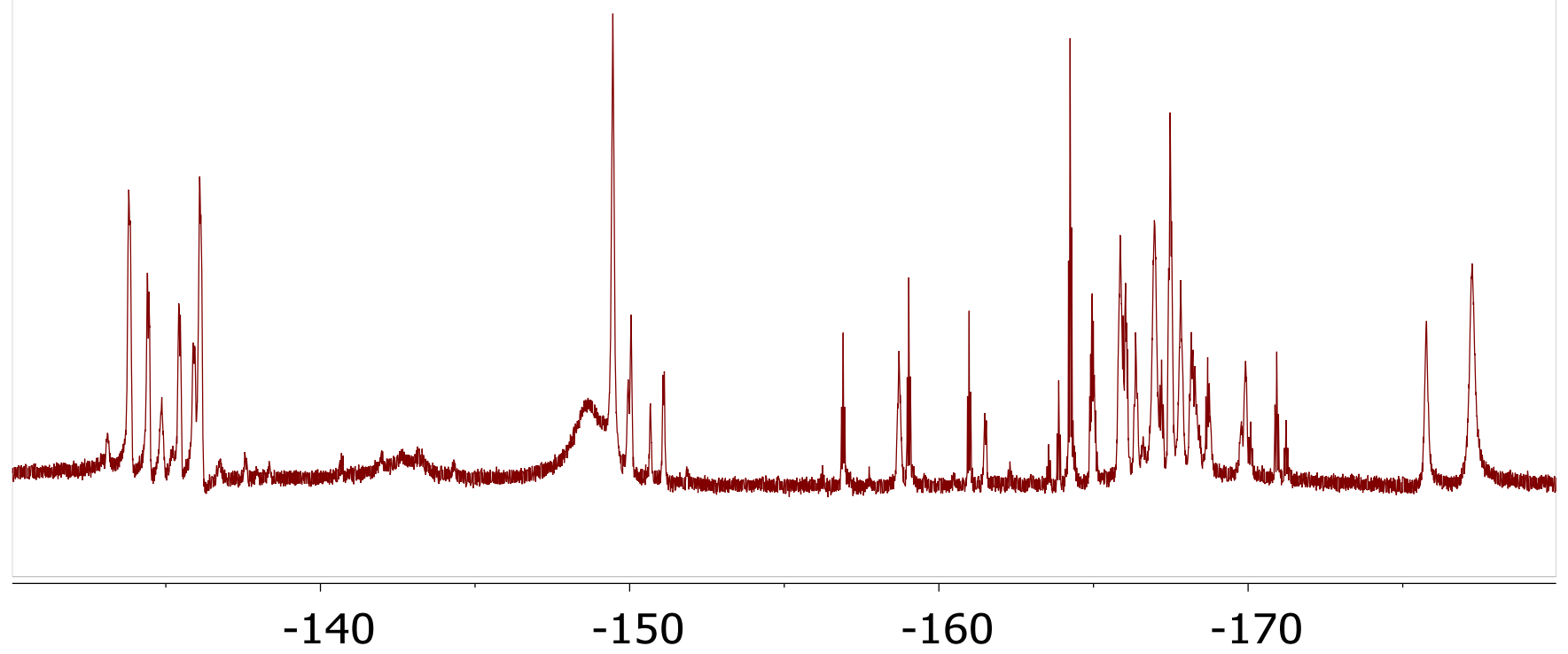

Figure S61. Crude ${ }^{19} \mathrm{~F}\left\{{ }^{1} \mathrm{H}\right\}$ NMR spectrum from the reaction between $3 \mathbf{b}^{\prime}$ with $\mathrm{Ph} 3 \mathrm{SnH}_{\mathrm{S}}$ showing multiple unidentified species. (376 MHz, $\mathrm{CD}_{3} \mathrm{CN}, 25^{\circ} \mathrm{C}$ ). 


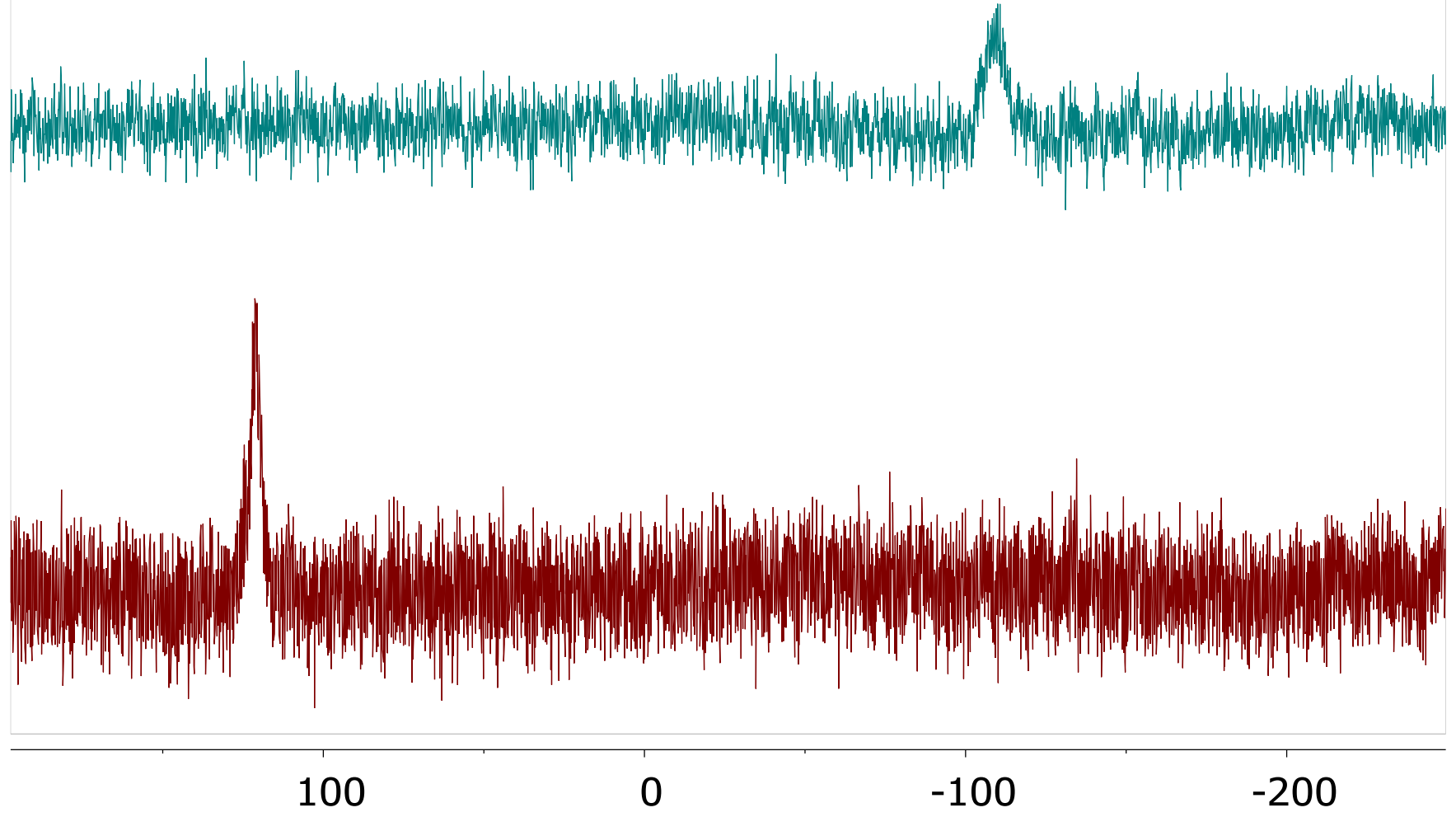

Figure S62. (bottom) ${ }^{51} \mathrm{~V}$ NMR spectrum of 1a. (top) ${ }^{51} \mathrm{~V}$ NMR spectrum of $\mathbf{1 a}$ after coordination of THF. (105 MHz, $\mathrm{C}_{6} \mathrm{D}_{6}, 25^{\circ} \mathrm{C}$ ). 


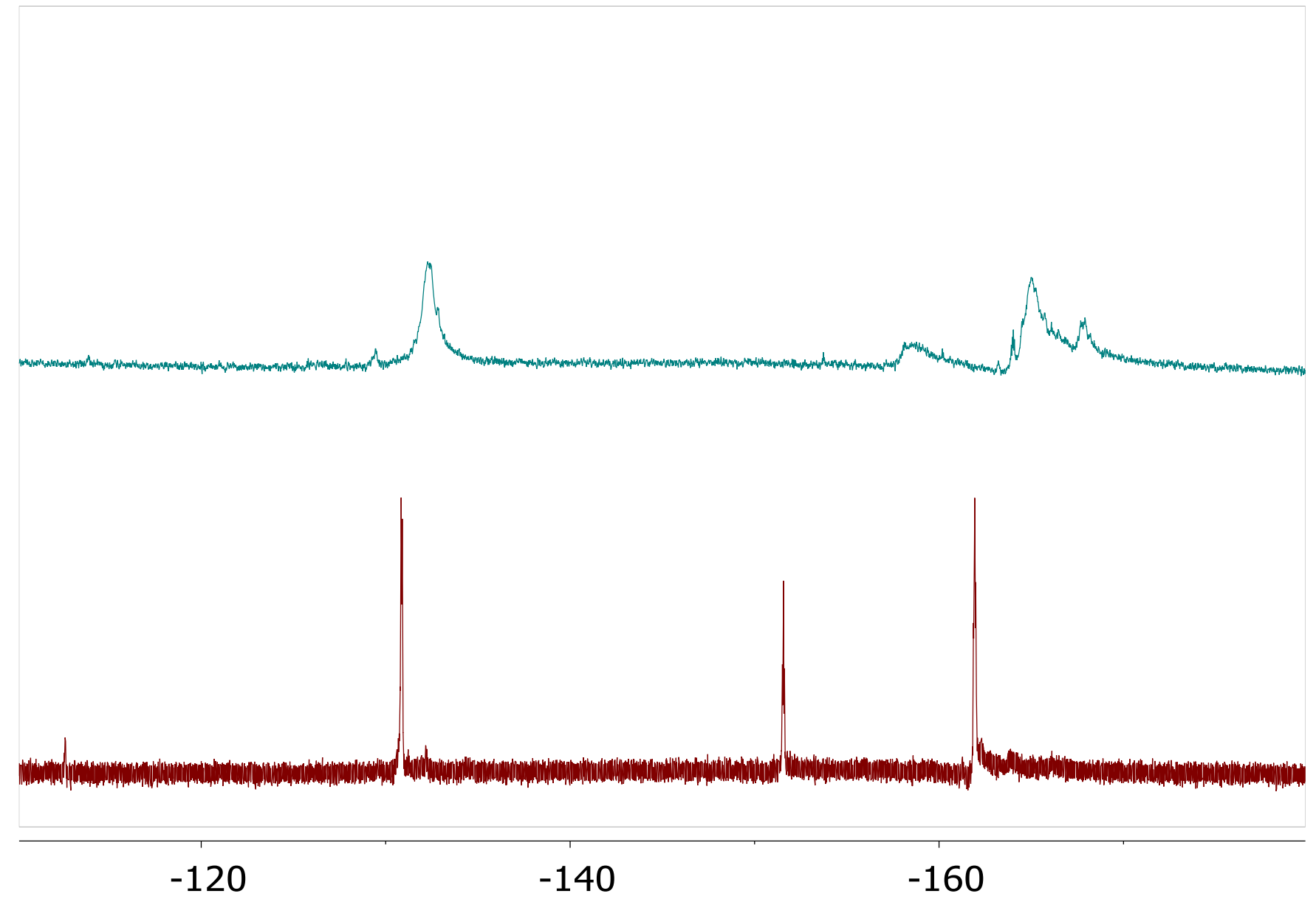

Figure S63. (bottom) ${ }^{19} \mathrm{~F}$ NMR spectrum of 1a. (top) ${ }^{19} \mathrm{~F}$ NMR spectrum of $\mathbf{1 a}$ after coordination of THF. (376 MHz, $\mathrm{C}_{6} \mathrm{D}_{6}, 25{ }^{\circ} \mathrm{C}$ ). 


\section{Supplemental Figures for EPR Spectroscopy}

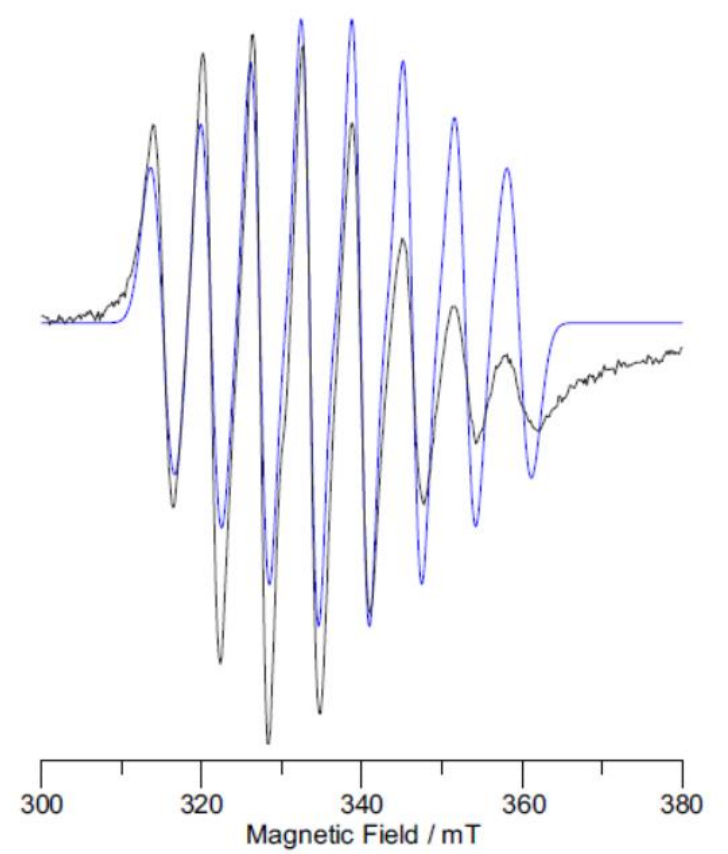

Figure S64. X-band EPR spectrum of 3a (benzene, $9.29542 \mathrm{GHz}, 298 \mathrm{~K}$ ). The experimental trace is in black and the simulation is in blue. Simulation parameters: $\boldsymbol{g}=1.9675, \boldsymbol{A}\left({ }^{51} \mathrm{~V}\right)=175 \mathrm{MHz}, W$ (Gaussian, hwhm $)=35 \mathrm{MHz}$. A hyperfine-dependent broadening term was included to model the observed line broadening. The model of Froncisz and Hyde was used ${ }^{1}$ as implemented in QPOW, in this case with their parameter $c_{1}=10 \mathrm{MHz}$.

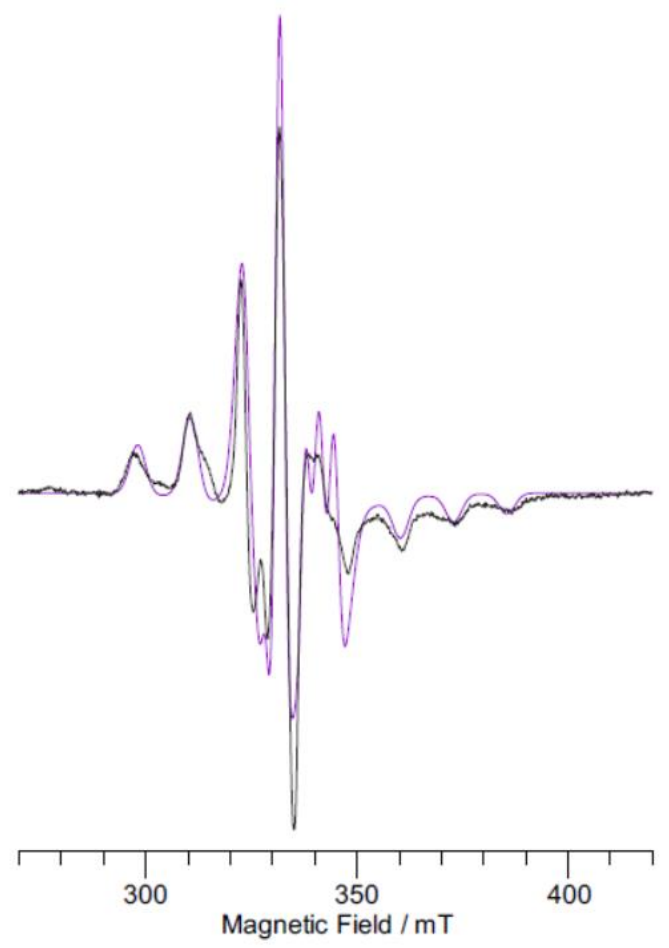

Figure S65. X-band EPR spectrum of 3a (THF, $9.29340 \mathrm{GHz}, 100 \mathrm{~K})$. The experimental trace is in black and the simulation is in purple. Simulation parameters: $\boldsymbol{g}=[1.98,1.98,1.942], \boldsymbol{A}\left({ }^{51} \mathrm{~V}\right)=[80,80,340]$ MHz, $W($ Gaussian, hwhm) $=40,40,60 \mathrm{MHz}$. 


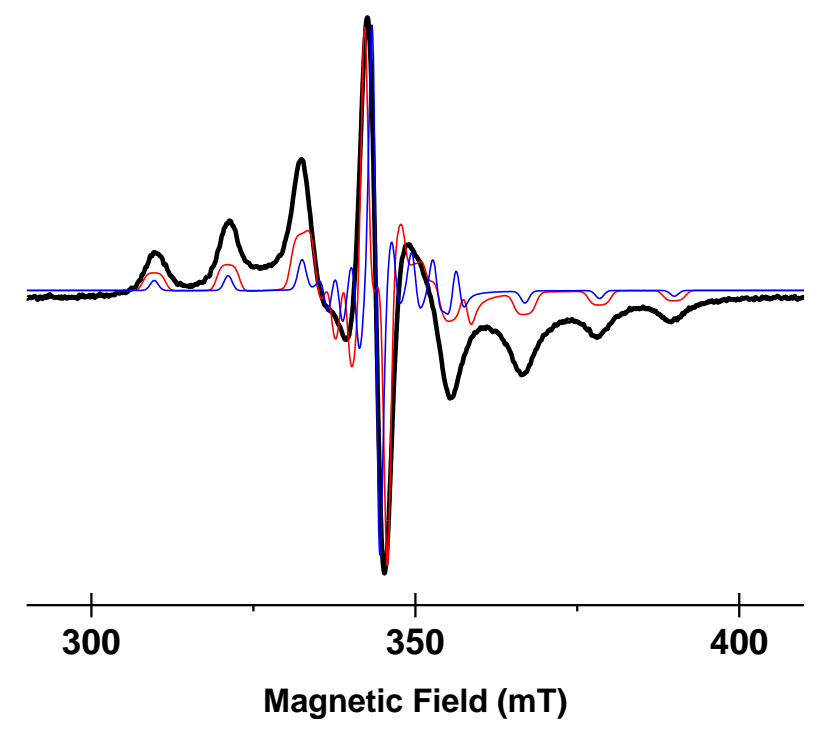

Figure S66. X-band EPR spectrum of 3b (black) with overlaid simulations in the absence of hyperfine coupling to ${ }^{11} \mathrm{~B}$ (blue) and in the presence of hyperfine coupling to ${ }^{11} \mathrm{~B}$ (red) (DCM, 9.6081 GHz, $100 \mathrm{~K}$ ). Simulation parameters: $\boldsymbol{g}=[1.98,1.98,1.962], \boldsymbol{A}\left({ }^{51} \mathrm{~V}\right)=[80,80,315] \mathrm{MHz}, W($ Gaussian, hwhm $)=20$ $\mathrm{MHz}$.

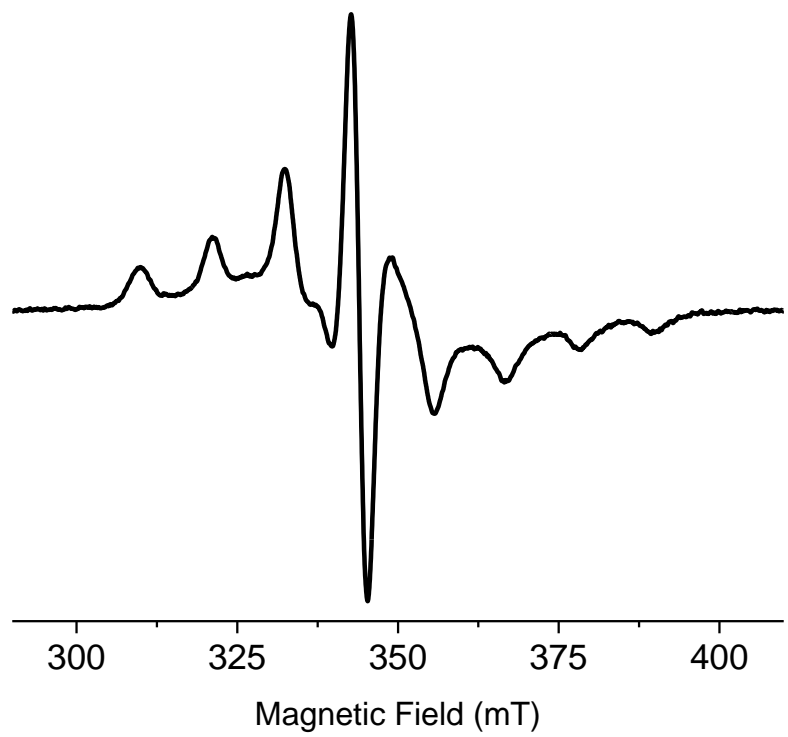

Figure S67. X-band EPR spectrum of 3b' (DCM, 9.6064 GHz, $100 \mathrm{~K}$ ). 


\section{Supplemental Figures for X-ray crystallography}

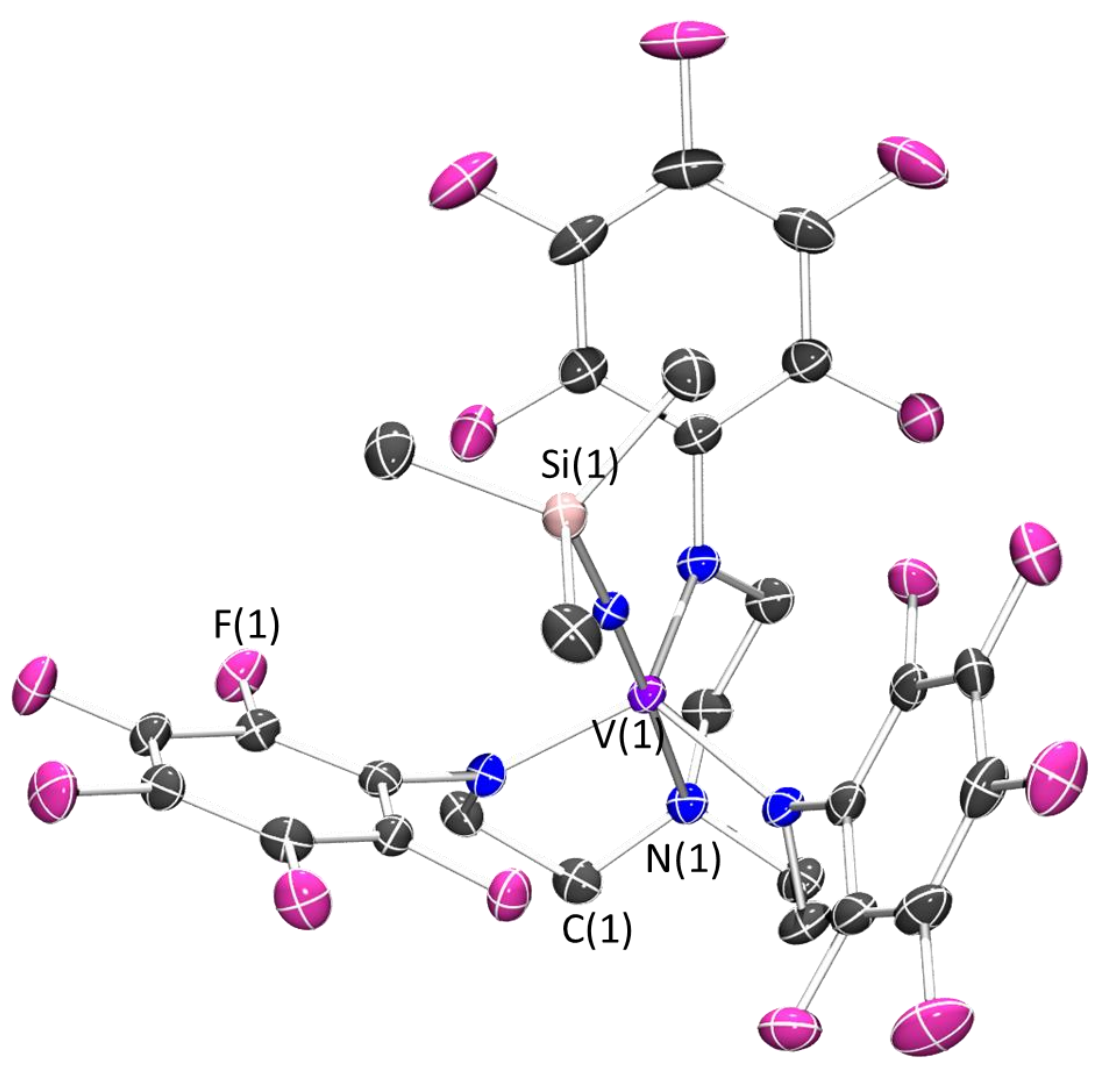

Figure S68. Solid-state molecular structure of (tren)VNTMS. All hydrogen atoms are omitted for clarity. 


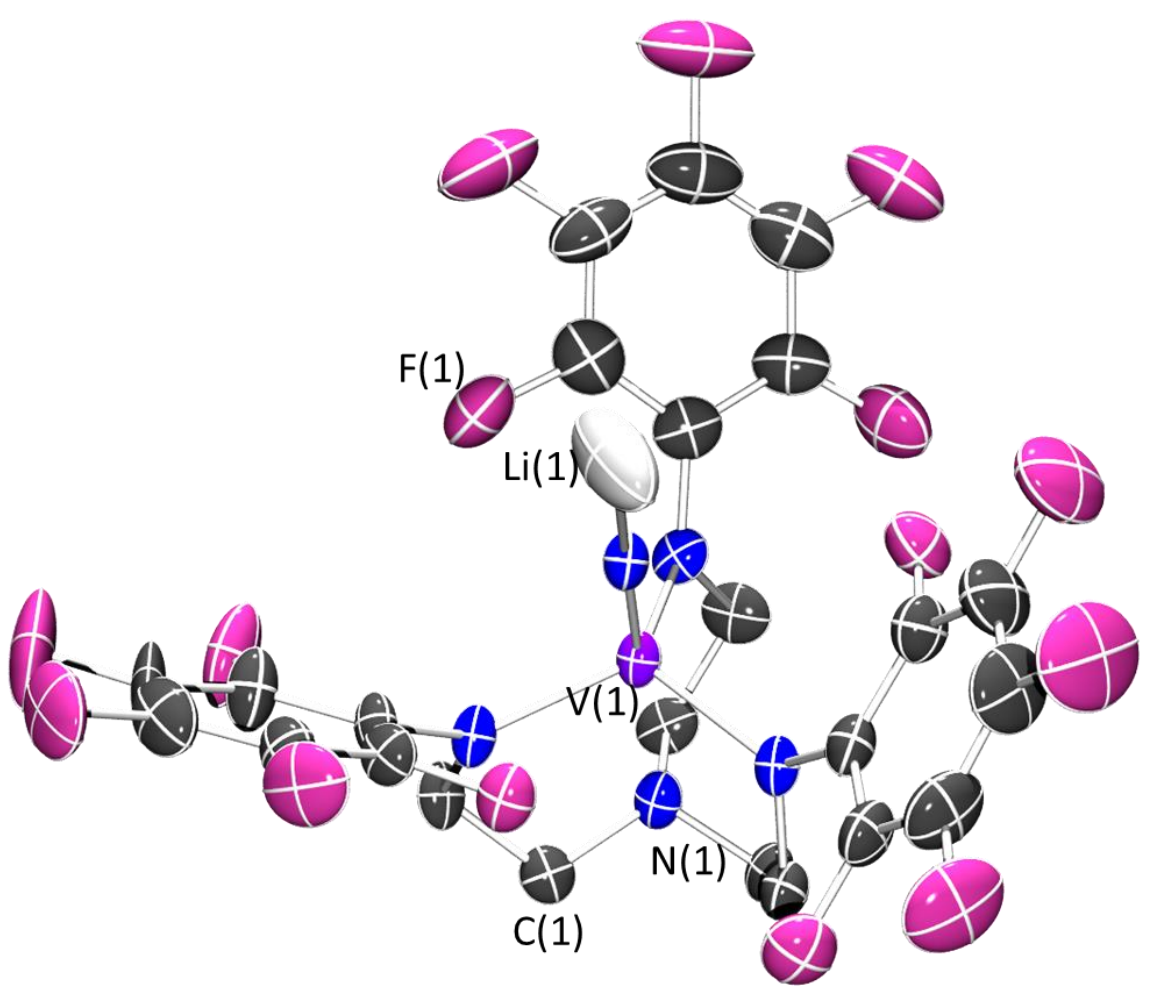

Figure S69. Solid-state molecular structure of (tren)VNLi. Disordered THF molecules on Li(1) and hydrogen atoms are omitted for clarity. 


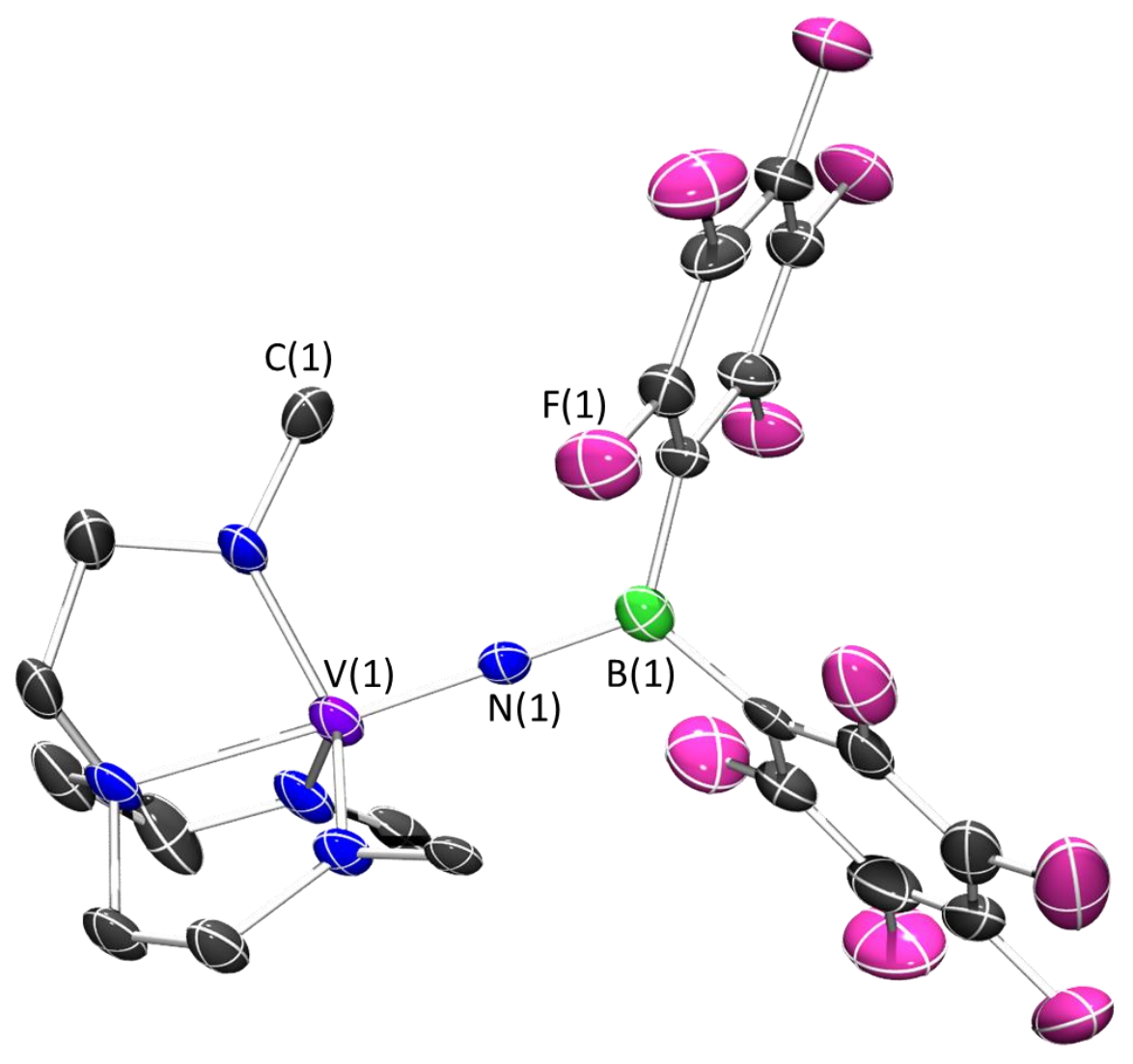

Figure S70. Solid-state molecular structure of $\mathbf{1 b}$. $\mathrm{C}_{6} \mathrm{~F}_{5}$ groups on tren ligand (except ipso carbons) and hydrogen atoms are omitted for clarity. 


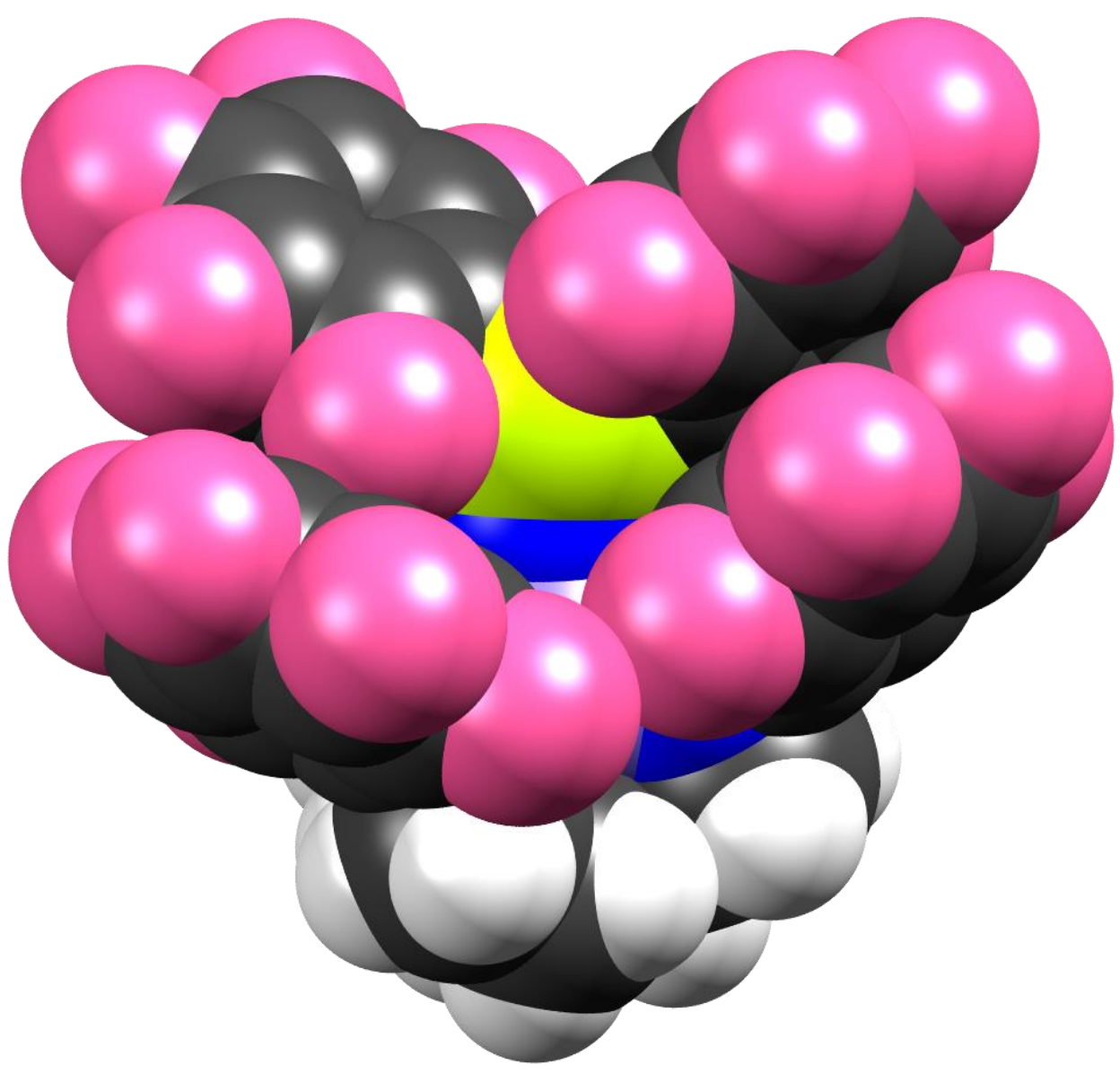

Figure S71. Space-filling model of $\mathbf{1 b}$ showing extreme steric crowding at $\mathrm{B}$. $(\mathrm{V}=$ purple, $\mathrm{N}=$ blue, $\mathrm{C}=$ black, $\mathrm{F}=$ pink, $\mathrm{B}=$ lime green, $\mathrm{H}=$ white) 


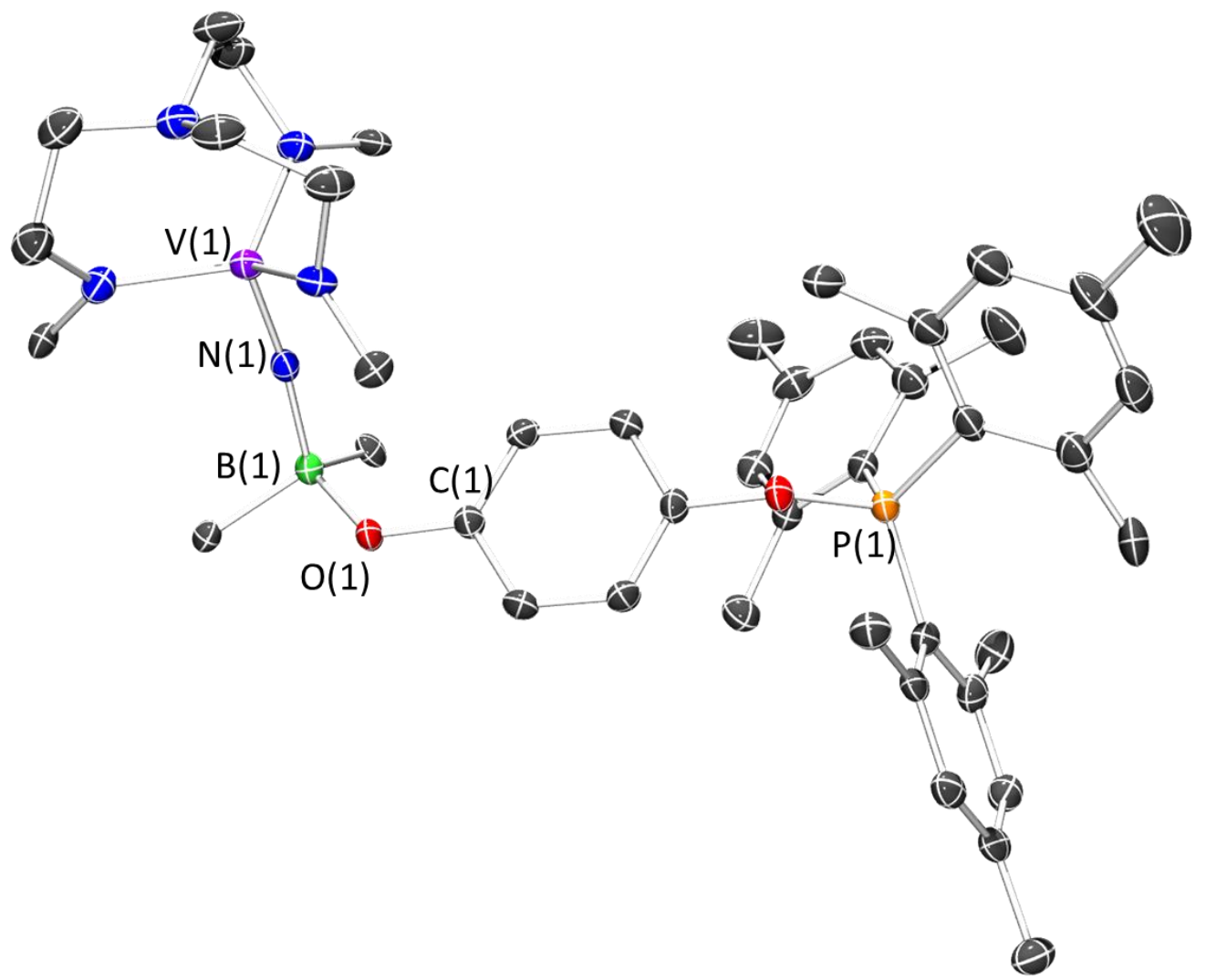

Figure S72. Solid-state molecular structure of $\mathbf{2}-\mathbf{H}_{4}$. All $\mathrm{C}_{6} \mathrm{~F}_{5}$ groups (except ipso carbons), cocrystallized solvent molecules, and hydrogen atoms are omitted for clarity. 


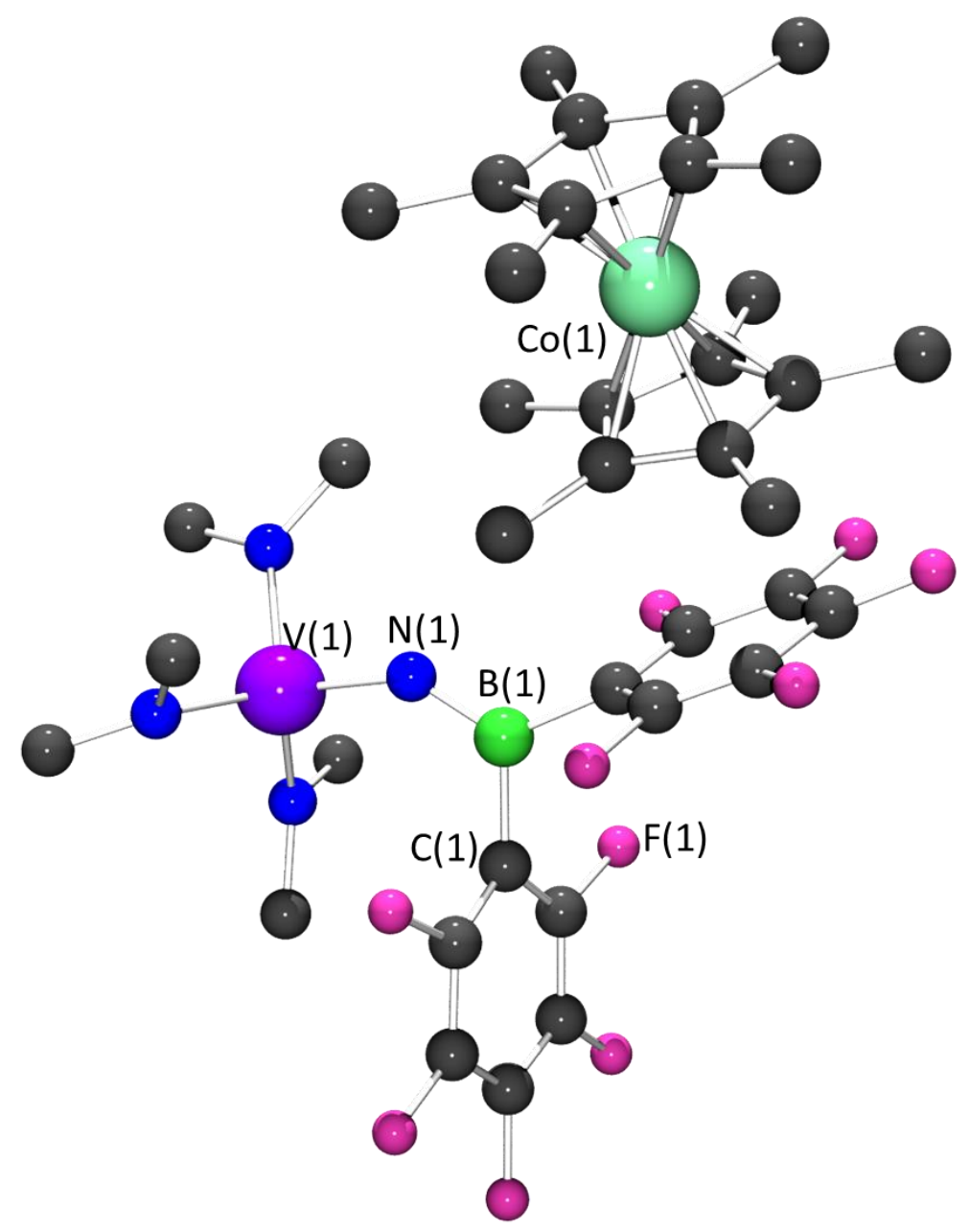

Figure S73. Low-resolution solid-state molecular structure of 3a. $\mathrm{NPh}_{2}$ groups (except ipso carbons) and hydrogen atoms are omitted for clarity. 


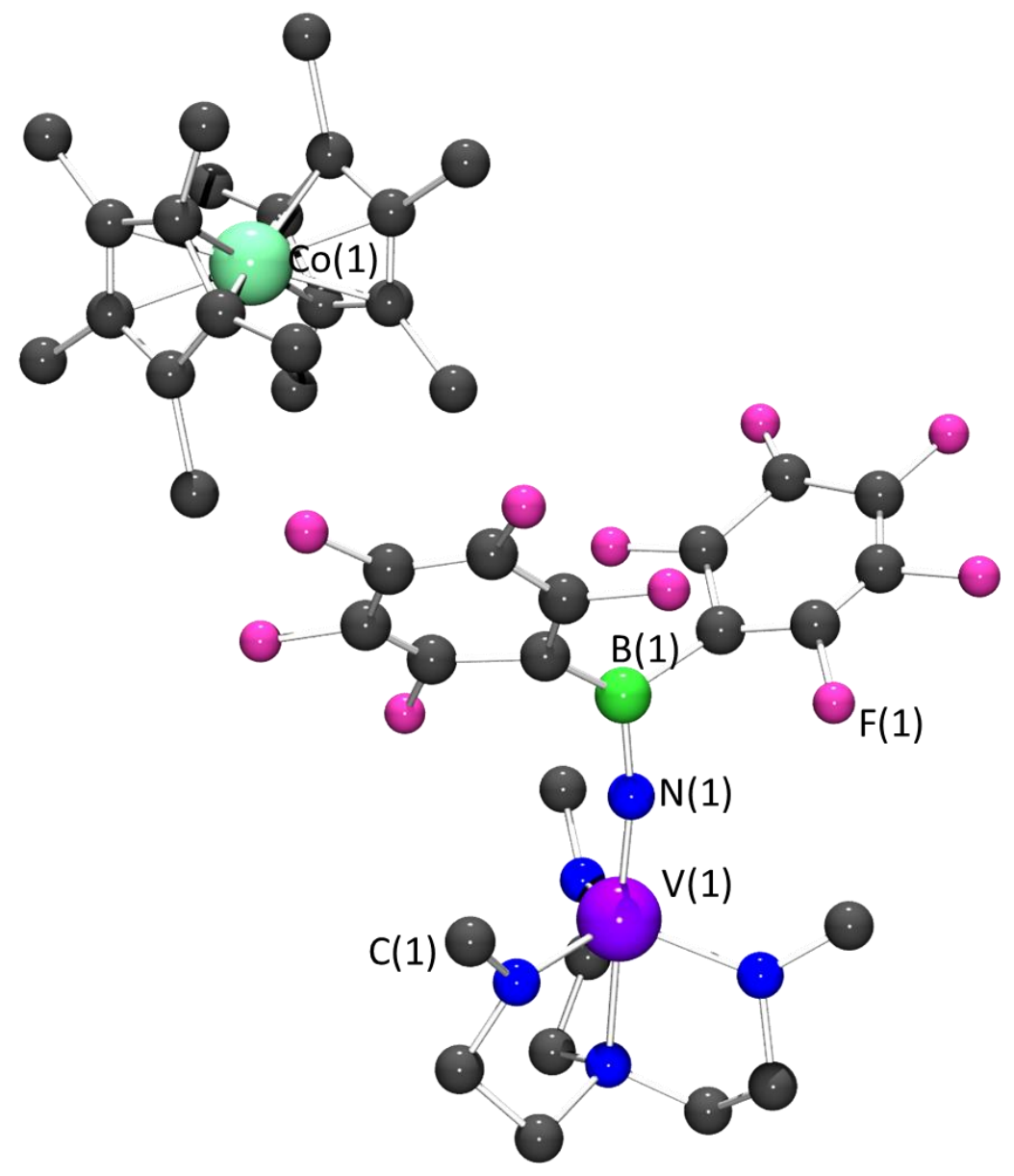

Figure S74. Low-resolution solid-state molecular structure of $\mathbf{3 b} . \mathrm{C}_{6} \mathrm{~F}_{5}$ groups on tren ligand (except ipso carbons) and hydrogen atoms are omitted for clarity. 


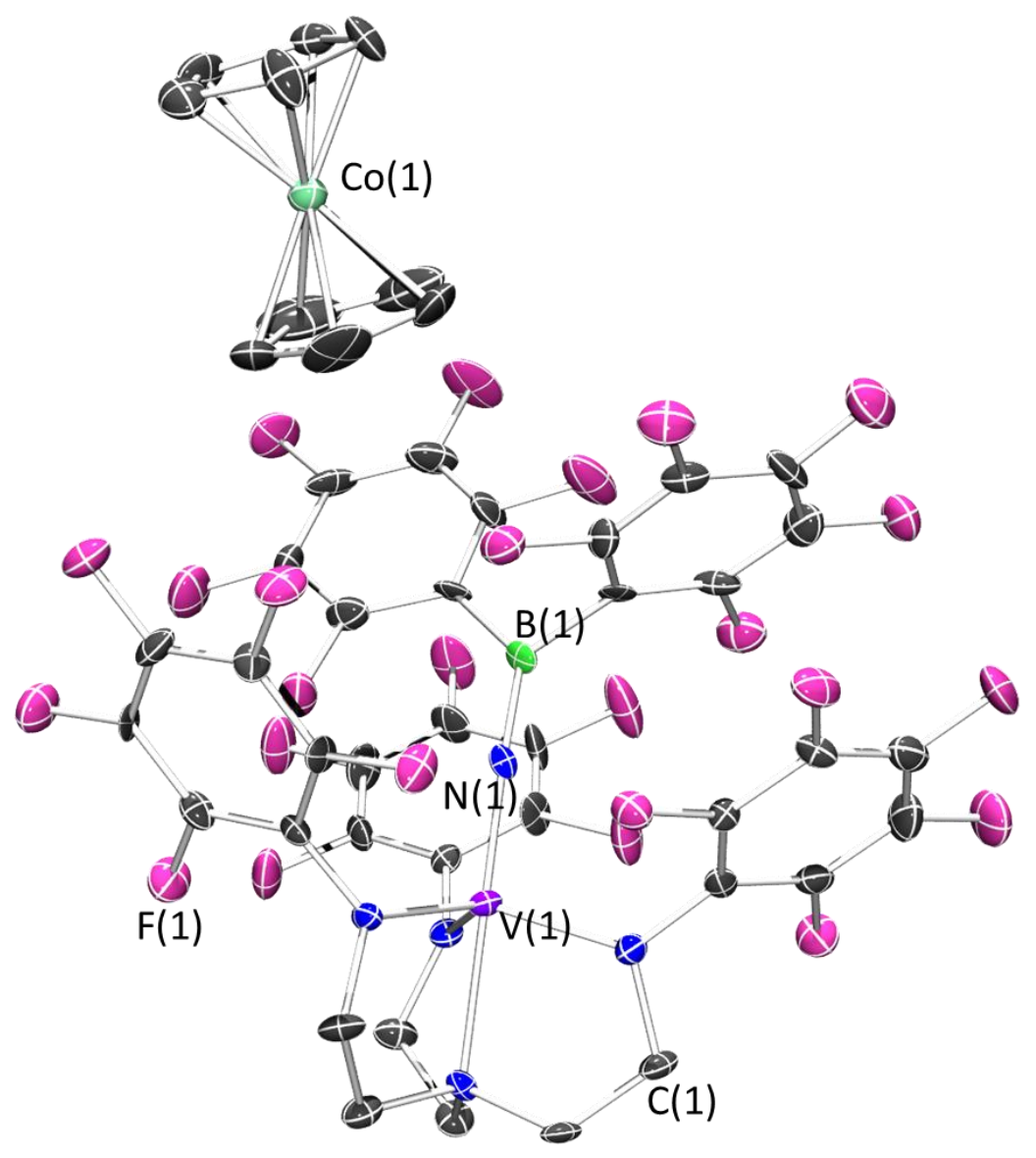

Figure S75. Solid-state molecular structure of $\mathbf{3 b}^{\prime}$. All hydrogen atoms are omitted for clarity. 


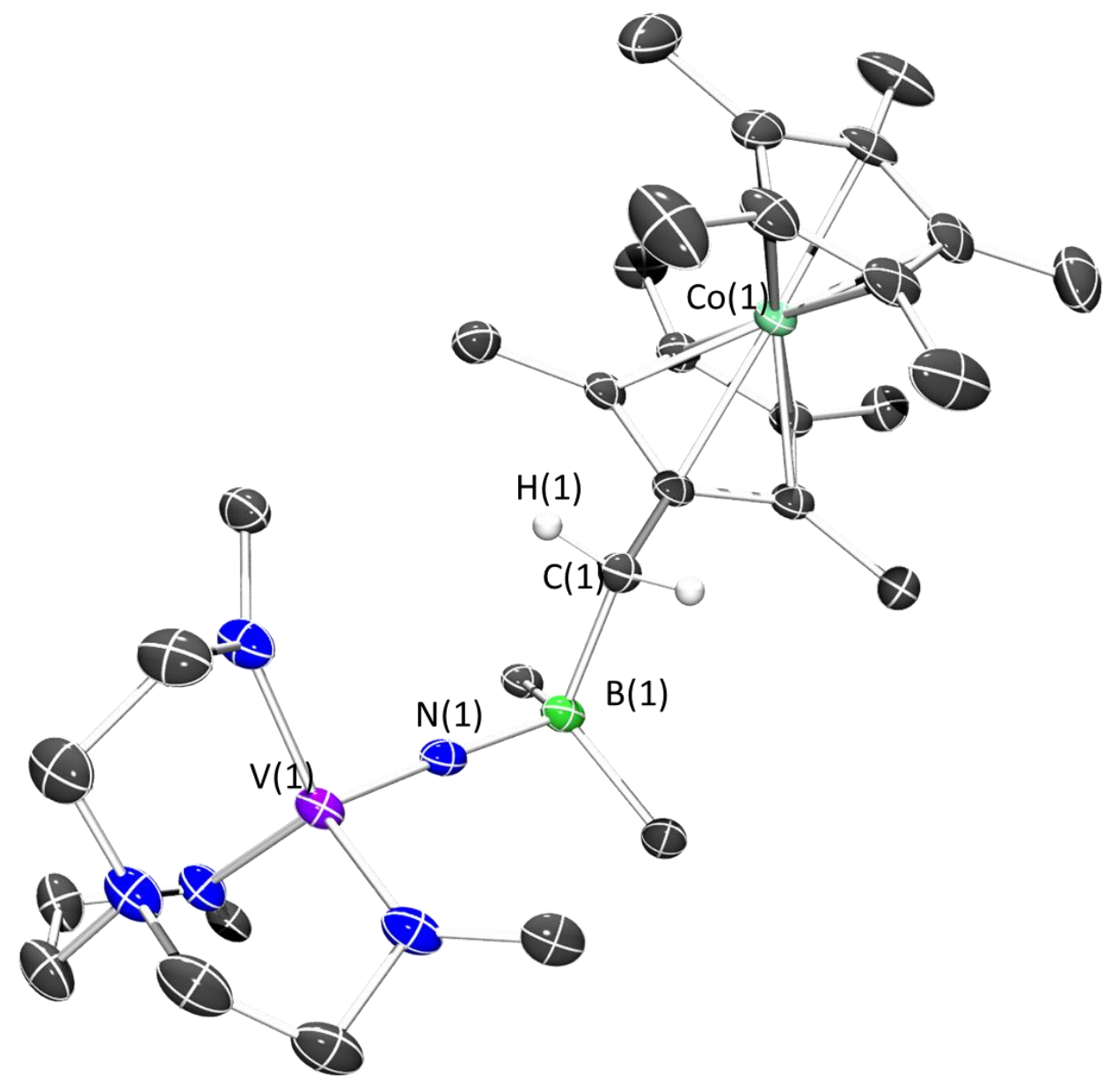

Figure S76. Solid-state molecular structure of $\mathbf{4 b}$. All $\mathrm{C}_{6} \mathrm{~F}_{5}$ groups (except ipso carbons) and hydrogen atoms (except those on $\mathrm{BCH}_{2}$ ) are omitted for clarity. 


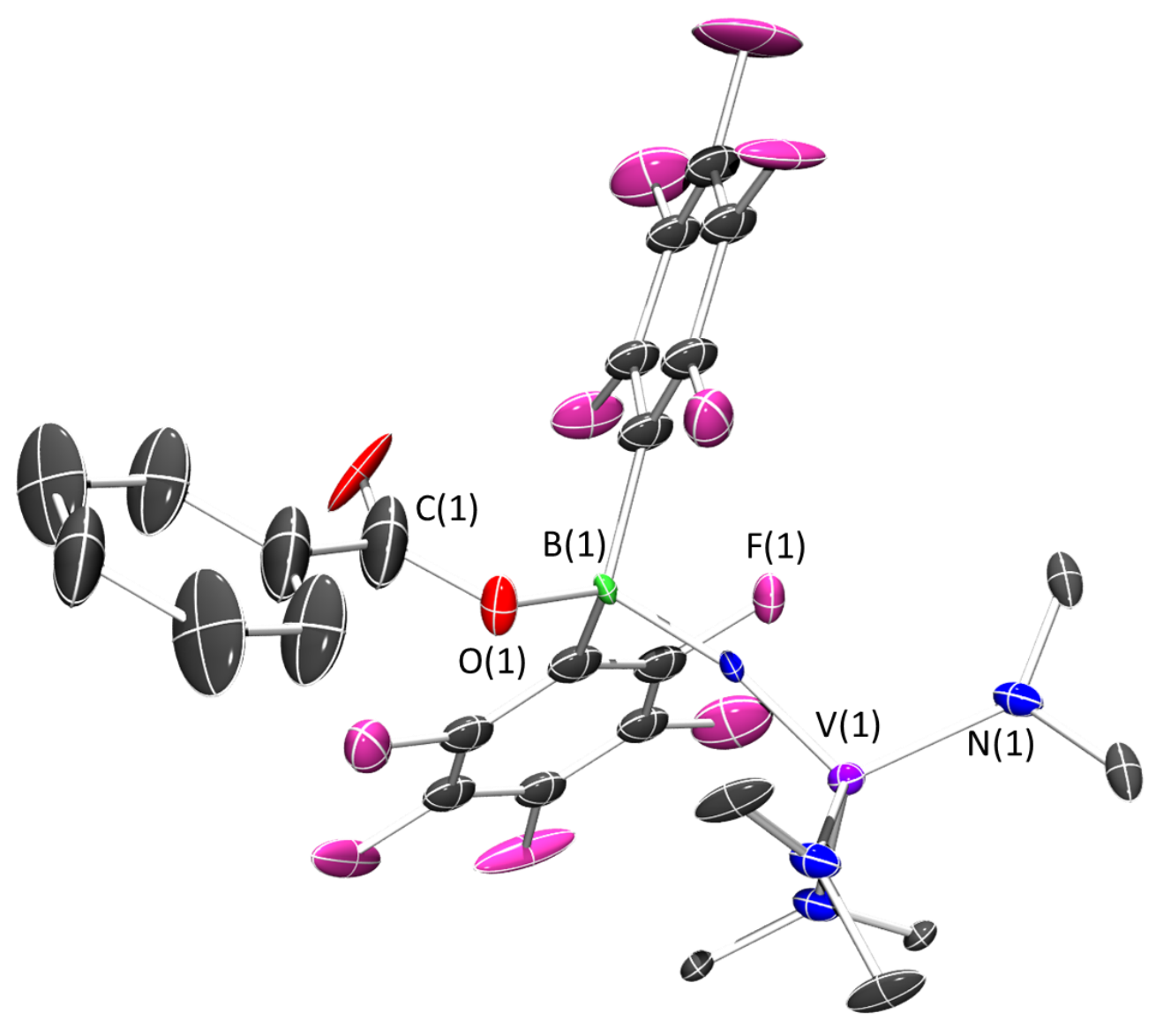

Figure S77. Solid-state molecular structure of 5a. $\mathrm{NPh}_{2}$ ligands (except ipso carbons), disordered $\left[\mathrm{CoCp}_{2}^{*}\right]^{+}$, and hydrogen atoms are omitted for clarity. 


\section{Supplemental Figures for Cyclic Voltammetry}

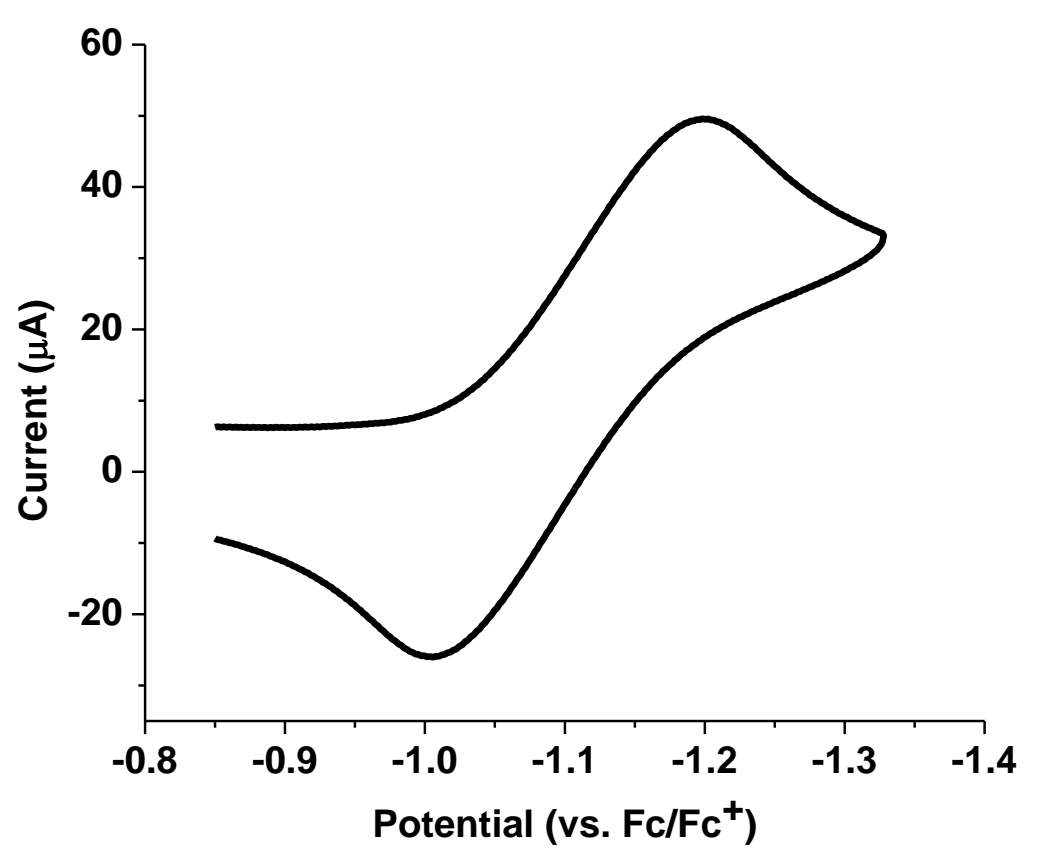

Figure S78. $\mathrm{CV}$ of $\mathbf{1 b}$ in $\mathrm{DCM}(3.0 \mathrm{mM})$ with $0.1 \mathrm{M}\left[\mathrm{Bu}_{4} \mathrm{~N}\right]\left[\mathrm{PF}_{6}\right]$ electrolyte (glassy carbon working electrode, $\mathrm{Ag}$ wire reference, platinum wire counter, scan rate $250 \mathrm{mV} / \mathrm{s}$, referenced to $\mathrm{Fc} / \mathrm{Fc}^{+}$). 


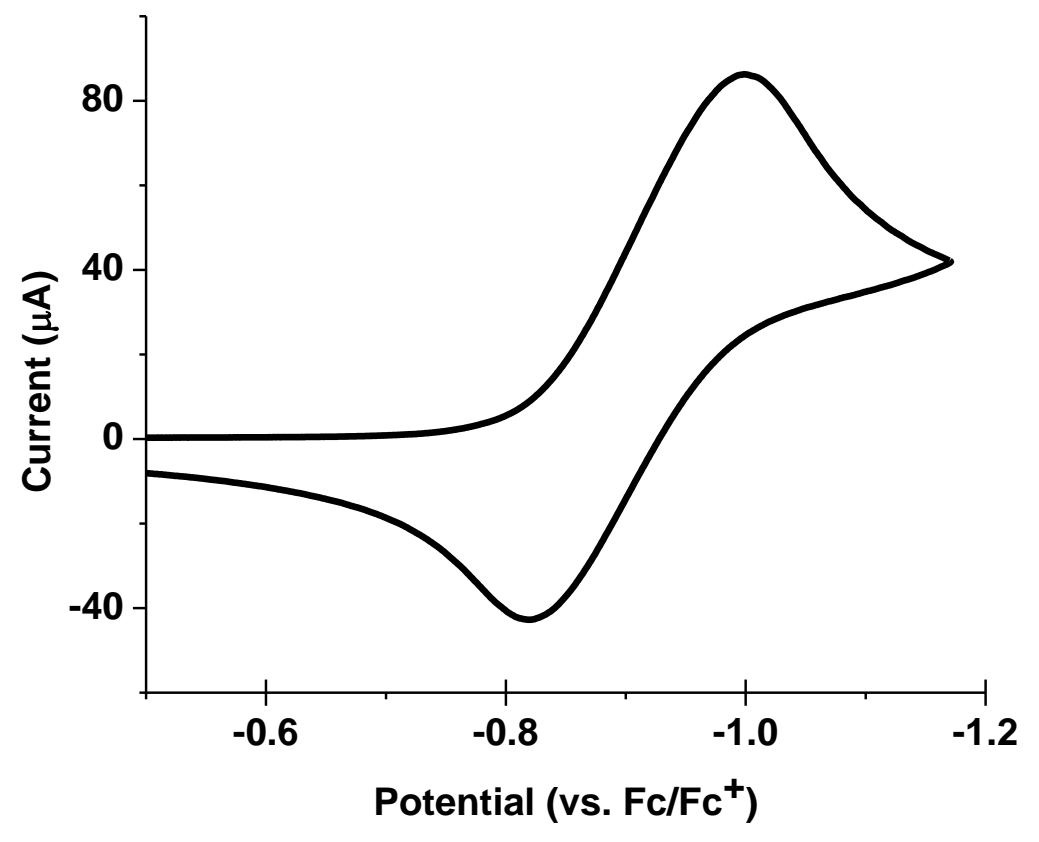

Figure S79. $\mathrm{CV}$ of $2,4,6-{ }^{t} \mathrm{Bu}_{3} \mathrm{C}_{6} \mathrm{H}_{2} \mathrm{O} \cdot$ in $\mathrm{DCM}(3.0 \mathrm{mM})$ with $0.1 \mathrm{M}\left[\mathrm{Bu}_{4} \mathrm{~N}\right]\left[\mathrm{PF}_{6}\right]$ electrolyte (glassy carbon working electrode, $\mathrm{Ag}$ wire reference, platinum wire counter, scan rate $250 \mathrm{mV} / \mathrm{s}$, referenced to $\left.\mathrm{Fc} / \mathrm{Fc}^{+}\right)$. 


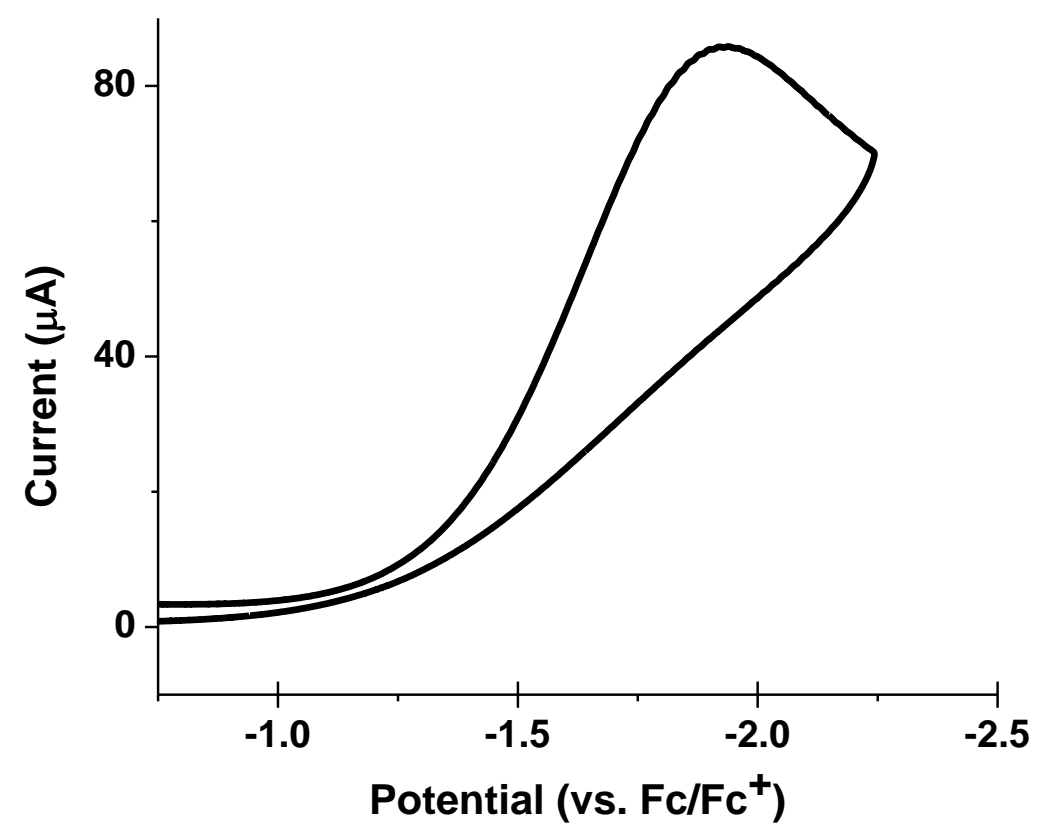

Figure S80. $\mathrm{CV}$ of benzoyl peroxide $(\mathrm{PhC}(\mathrm{O}) \mathrm{OOC}(\mathrm{O}) \mathrm{Ph})$ in $\mathrm{DCM}(3.0 \mathrm{mM})$ with $0.1 \mathrm{M}\left[\mathrm{Bu}_{4} \mathrm{~N}\right]\left[\mathrm{PF}_{6}\right]$ electrolyte (glassy carbon working electrode, $\mathrm{Ag}$ wire reference, platinum wire counter, scan rate 250 $\mathrm{mV} / \mathrm{s}$, referenced to $\mathrm{Fc} / \mathrm{Fc}^{+}$). 


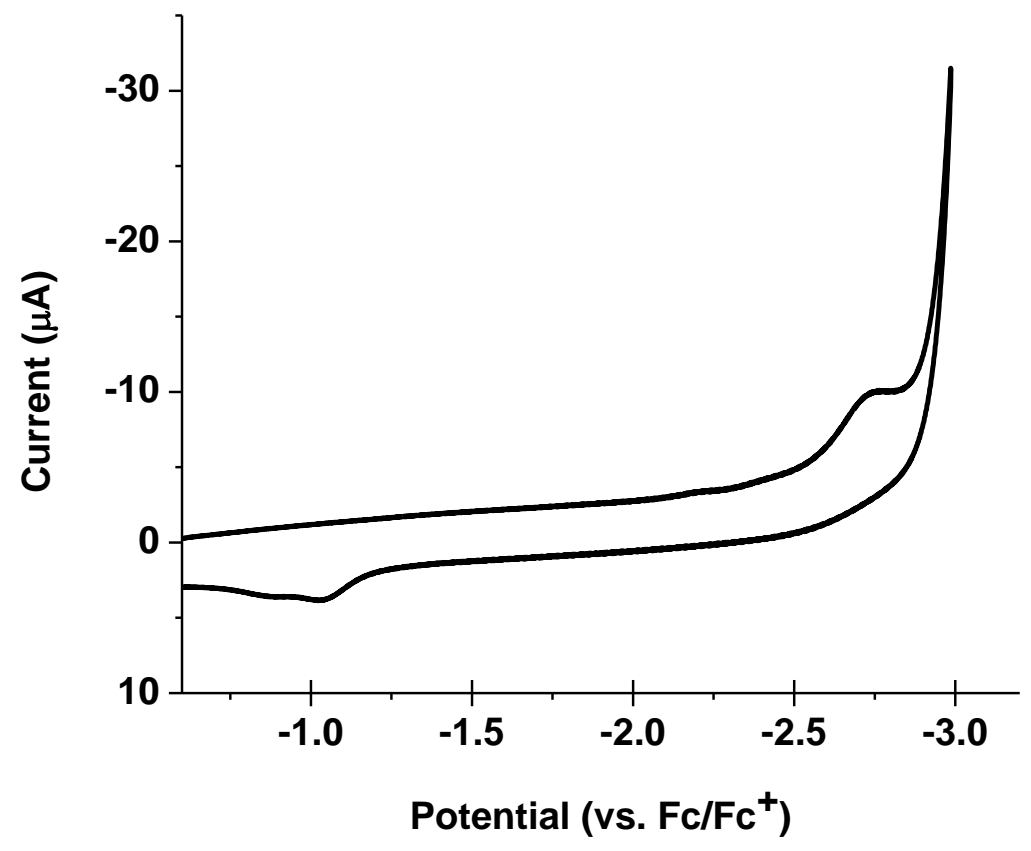

Figure S81. $\mathrm{CV}$ of $\mathrm{Ph}_{3} \mathrm{SnH}$ in acetonitrile $(3.0 \mathrm{mM})$ with $0.1 \mathrm{M}\left[\mathrm{Bu}_{4} \mathrm{~N}\right]\left[\mathrm{PF}_{6}\right]$ electrolyte (glassy carbon working electrode, $\mathrm{Ag}$ wire reference, platinum wire counter, scan rate $250 \mathrm{mV} / \mathrm{s}$, referenced to $\mathrm{Fc} / \mathrm{Fc}^{+}$). An irreversible reduction event at $-2.7 \mathrm{~V}$ is assigned to $\mathrm{Ph}_{3} \mathrm{SnH}^{0 / \bullet-}$ that subsequently undergoes an unknown chemical reaction with a corresponding oxidative feature at $\sim-1.0 \mathrm{~V}$. These features are not observed when scanning to less than $-2.7 \mathrm{~V}$ (see Figure S82). 


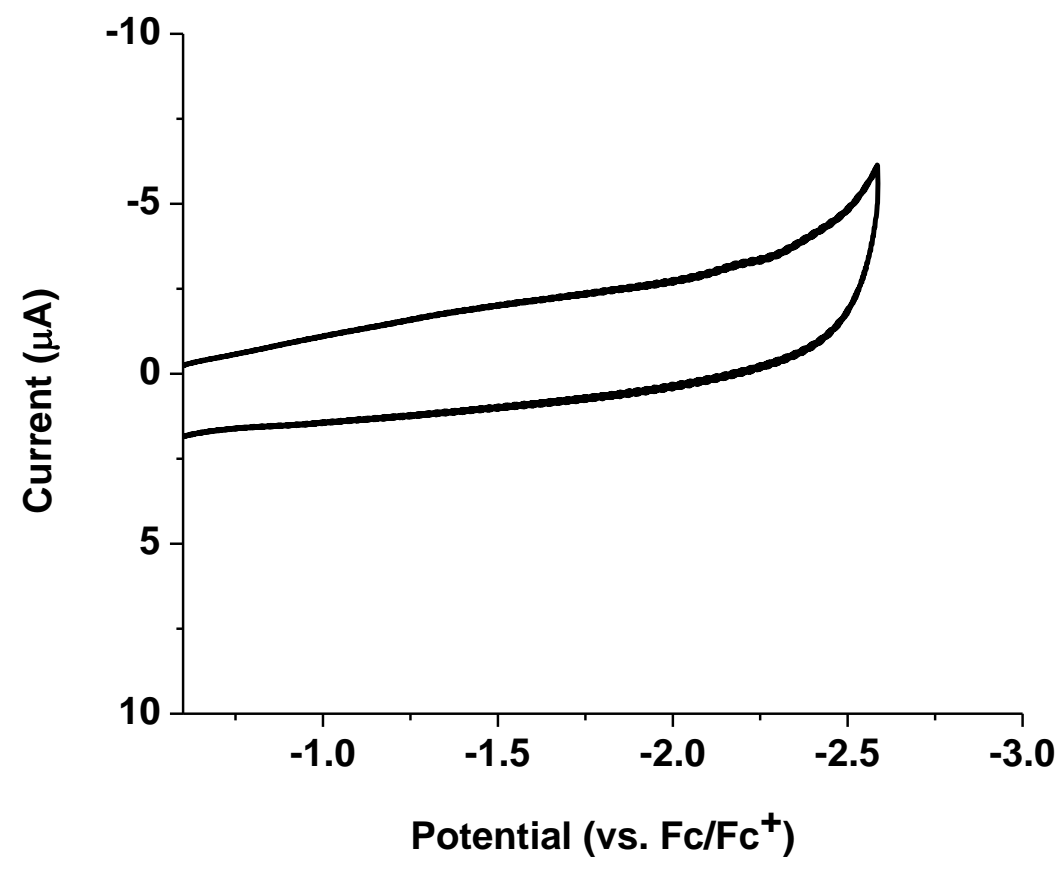

Figure S82. $\mathrm{CV}$ of $\mathrm{Ph}_{3} \mathrm{SnH}$ in acetonitrile $(3.0 \mathrm{mM})$ with $0.1 \mathrm{M}\left[\mathrm{Bu}_{4} \mathrm{~N}\right]\left[\mathrm{PF}_{6}\right]$ electrolyte (glassy carbon working electrode, $\mathrm{Ag}$ wire reference, platinum wire counter, scan rate $250 \mathrm{mV} / \mathrm{s}$, referenced to $\mathrm{Fc} / \mathrm{Fc}^{+}$). 


\section{Supplemental Table}

Table S1. Selected bond lengths $(\AA)$ and bond angles $\left({ }^{\circ}\right)$.

\begin{tabular}{|c|c|c|c|c|c|}
\hline & 1a & $1 \mathbf{b}$ & $2-F_{4}$ & $2-\mathrm{H}_{4}$ & $3 \mathbf{a}$ \\
\hline Bond $^{[a]}$ & Distance $(\AA)$ & Distance $(\AA)$ & Distance $(\AA)$ & Distance $(\AA)$ & Distance $(\AA)$ \\
\hline $\mathrm{V}(1)-\mathrm{N}(4)$ & $1.670(6)$ & $1.703(4)$ & $1.653(7)$ & $1.654(3)$ & $1.885(8)$ \\
\hline$N(4)-B(1)$ & $1.421(11)$ & $1.428(6)$ & $1.532(12)$ & $1.566(5)$ & $1.368(14)$ \\
\hline Bond $^{[\mathrm{a}]}$ & Angle $\left(^{0}\right)$ & Angle $\left({ }^{0}\right)$ & Angle $\left({ }^{0}\right)$ & Angle $\left({ }^{0}\right)$ & Angle $\left({ }^{0}\right)$ \\
\hline $\mathrm{V}(1)-\mathrm{N}(4)-$ & $161.1(6)$ & $170.0(3)$ & $170.9(6)$ & $171.0(3)$ & 139.2(9) \\
\hline & $3 \mathbf{b}^{\prime}$ & $4 a$ & $4 b$ & $5 \mathbf{a}$ & $5 b$ \\
\hline Bond $^{[\mathrm{a}]}$ & Distance $(\AA)$ & Distance $(\AA)$ & Distance $(\AA)$ & Distance $(\AA)$ & Distance $(\AA)$ \\
\hline $\mathrm{V}(1)-\mathrm{N}(4)$ & $1.776(4)$ & $\begin{array}{l}1.631(6) \\
1.639(6)\end{array}$ & $1.652(2)$ & $1.622(12)$ & $1.654(13)$ \\
\hline $\mathrm{N}(4)-\mathrm{B}(1)$ & $1.354(7)$ & $\begin{array}{c}1.571(10) \\
1.542(9)\end{array}$ & $1.558(4)$ & $1.60(2)$ & $1.60(2)$ \\
\hline Bond $^{[\mathrm{a}]}$ & Angle $\left({ }^{0}\right)$ & Angle $\left({ }^{0}\right)$ & Angle $\left({ }^{0}\right)$ & Angle $\left({ }^{0}\right)$ & Angle $\left({ }^{0}\right)$ \\
\hline $\begin{array}{c}\mathrm{V}(1)-\mathrm{N}(4)- \\
\mathrm{B}(1)\end{array}$ & $173.9(4)$ & $\begin{array}{l}166.1(5) \\
167.0(5)\end{array}$ & $175.4(19)$ & $163.6(10)$ & $174.5(11)$ \\
\hline
\end{tabular}

[a] for tren-containing compounds, $\mathrm{N}(4)$ is replaced with $\mathrm{N}(5)$ 
6. DFT Results

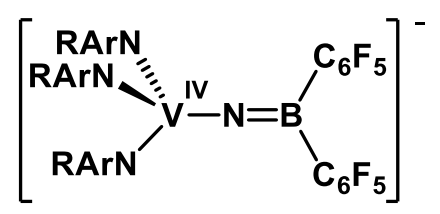

$\begin{array}{lrrr}\text { V } & -0.07683 & -1.97189 & -0.71484 \\ \text { F } & 2.84042 & -2.19923 & 1.20271 \\ \text { F } & 0.36471 & 1.68270 & 3.07717 \\ \text { F } & 0.76825 & -1.57781 & 2.83383 \\ \text { F } & -3.03566 & -0.80742 & 5.42058 \\ \text { F } & 2.74743 & 1.69184 & -0.97156 \\ \text { F } & 3.24900 & -1.43373 & -3.42638 \\ \text { F } & 5.16531 & -0.95931 & 1.60298 \\ \text { F } & -0.37065 & -0.56955 & 5.01855 \\ \text { F } & 2.45753 & 2.82115 & 4.30621 \\ \text { F } & -2.59639 & 0.41715 & 1.08194 \\ \text { F } & -4.10278 & -0.73897 & -1.95929 \\ \text { F } & 4.82233 & 2.84127 & 0.25787 \\ \text { F } & 0.33898 & 0.21538 & -3.21311 \\ \text { F } & -3.43443 & -3.11725 & 1.37663 \\ \text { F } & -4.55835 & -2.10486 & 3.58406 \\ \text { F } & 4.69566 & 3.41266 & 2.90158 \\ \text { F } & 6.59868 & -0.00845 & -0.49480 \\ \text { F } & -4.18906 & 4.70204 & 0.20851 \\ \text { F } & -4.85736 & 1.65209 & -2.86940 \\ \text { F } & -3.02970 & 3.37783 & -3.89886 \\ \text { F } & 5.61542 & -0.27958 & -3.01343 \\ \text { F } & -4.59062 & 2.16733 & 1.07428 \\ \text { F } & -0.42797 & 2.62906 & -4.04595 \\ \text { F } & -1.73443 & 5.45463 & -0.66034 \\ \text { F } & 0.28000 & 3.71785 & -0.65884 \\ \text { N } & -0.48236 & -4.07606 & -1.58726 \\ \text { N } & 1.71861 & -2.50464 & -1.32001 \\ \text { N } & 0.14523 & -0.32165 & -0.12441 \\ \text { N } & -0.73494 & -2.91630 & 0.83533 \\ \text { N } & -1.42142 & -1.61562 & -2.10967 \\ \text { C } & -1.28626 & -2.36978 & 1.99297 \\ \text { C } & 3.46772 & -1.69020 & 0.14968 \\ \text { C } & 2.91842 & -1.84936 & -1.12489 \\ \text { C } & -0.54311 & -1.70113 & 2.96359 \\ \text { C } & -3.16898 & 0.05890 & -2.48693 \\ \text { C } & -2.65121 & -2.48878 & 2.25857 \\ \text { C } & -3.24673 & -1.97367 & 3.38939 \\ \text { C } & -1.84019 & -0.36766 & -2.52545 \\ \text { C } & 3.69768 & -1.34762 & -2.17073 \\ \text { C } & 1.46282 & 1.93113 & 2.35473 \\ \text { C } & 1.48527 & 1.60119 & 1.01203 \\ \text { C } & -1.12196 & -1.17443 & 4.10375 \\ \text { C } & 2.64673 & 1.92565 & 0.33714 \\ \text { C } & -0.93768 & 0.55054 & -3.07486 \\ & & & \end{array}$




$\begin{array}{lrrr}\mathrm{C} & -1.05410 & 1.97761 & 0.21473 \\ \mathrm{C} & 1.76090 & -3.55501 & -2.33061 \\ \mathrm{H} & 2.77242 & -3.95681 & -2.43251 \\ \mathrm{H} & 1.46469 & -3.19619 & -3.32065 \\ \mathrm{C} & -2.33259 & 1.64281 & 0.63199 \\ \mathrm{C} & 4.68091 & -1.06896 & 0.36659 \\ \mathrm{C} & 5.42100 & -0.59483 & -0.69761 \\ \mathrm{C} & 2.52284 & 2.52706 & 3.00852 \\ \mathrm{C} & 3.66488 & 2.82974 & 2.29568 \\ \mathrm{C} & 0.83489 & -4.65797 & -1.86783 \\ \mathrm{H} & 0.75402 & -5.46806 & -2.60288 \\ \mathrm{H} & 1.22430 & -5.07204 & -0.93977 \\ \mathrm{C} & -2.21684 & -2.72918 & -2.60715 \\ \mathrm{H} & -2.68868 & -2.47868 & -3.56128 \\ \mathrm{H} & -3.02297 & -3.00362 & -1.92030 \\ \mathrm{C} & -1.32391 & 1.79599 & -3.52363 \\ \mathrm{C} & -2.47745 & -1.30634 & 4.32015 \\ \mathrm{C} & -1.27148 & -3.89014 & -2.81030 \\ \mathrm{H} & -1.80143 & -4.81300 & -3.07491 \\ \mathrm{H} & -0.58479 & -3.64454 & -3.61689 \\ \mathrm{C} & 3.72736 & 2.53149 & 0.94965 \\ \mathrm{C} & -0.76364 & -4.37517 & 0.80269 \\ \mathrm{H} & -1.43970 & -4.77923 & 1.55834 \\ \mathrm{H} & 0.23210 & -4.77715 & 1.02507 \\ \mathrm{C} & -1.22588 & -4.82147 & -0.56651 \\ \mathrm{H} & -1.11262 & -5.90289 & -0.70971 \\ \mathrm{H} & -2.27915 & -4.56958 & -0.67044 \\ \mathrm{C} & -0.90561 & 3.28897 & -0.21466 \\ \mathrm{C} & -3.38673 & 2.53688 & 0.64360 \\ \mathrm{C} & -3.18596 & 3.83047 & 0.20847 \\ \mathrm{C} & -3.57546 & 1.29581 & -2.94740 \\ \mathrm{C} & 4.92215 & -0.74345 & -1.97439 \\ \mathrm{C} & -2.65056 & 2.17702 & -3.46404 \\ \mathrm{C} & -1.93372 & 4.21038 & -0.22919 \\ \mathrm{~B} & 0.19093 & 0.96650 & 0.30582 \\ & & & \end{array}$




\section{ELECTRONIC G-MATRIX}

The g-matrix:

$$
\begin{array}{rrr}
1.9699559 & -0.0015431 & -0.0010922 \\
-0.0015758 & 1.9776490 & 0.0060086 \\
-0.0015540 & 0.0058160 & 1.9798963
\end{array}
$$

\begin{tabular}{lrrrr} 
gel & 2.0023193 & 2.0023193 & 2.0023193 & \\
gRMC & -0.0005699 & -0.0005699 & -0.0005699 & \\
gDSO(tot) & 0.0003656 & 0.0003073 & 0.0002033 & \\
gPSO(tot) & -0.0324680 & -0.0292619 & -0.0168933 & \\
\multicolumn{1}{c}{ g(tot) } & 1.9696471 & 1.9727947 & 1.9850595 & iso $=1.9758338$ \\
Delta-g & -0.0326722 & -0.0295245 & -0.0172598 & iso $=-0.0264855$
\end{tabular}

Orientation:

$\begin{array}{llll}\mathrm{X} & 0.9848886 & 0.1117779 & 0.1322884 \\ \mathrm{Y} & 0.1707978 & -0.7533752 & -0.6350227 \\ \mathrm{Z} & 0.0286813 & 0.6480212 & -0.7610821\end{array}$

Notes:

(1) The principal values are square roots of the eigenvalues of $\mathrm{gT}^{*} \mathrm{~g}$

(2) Orientations are eigenvectors of $\mathrm{gT}^{*} \mathrm{~g}$ written as column vectors

(3) Individual contributions are projections of the full matrices onto the eigenvectors of $\mathrm{gT}^{*} \mathrm{~g}$

(4) Tensor is right-handed 
Nucleus B : A:ISTP= $11 \mathrm{I}=1.5 \mathrm{P}=171.2151 \mathrm{MHz} / \mathrm{au}^{* * 3}$

$\mathrm{Q}: \mathrm{ISTP}=11 \mathrm{I}=1.5 \mathrm{Q}=0.0410$ barn

Raw HFC matrix (all values in $\mathrm{MHz}$ ):

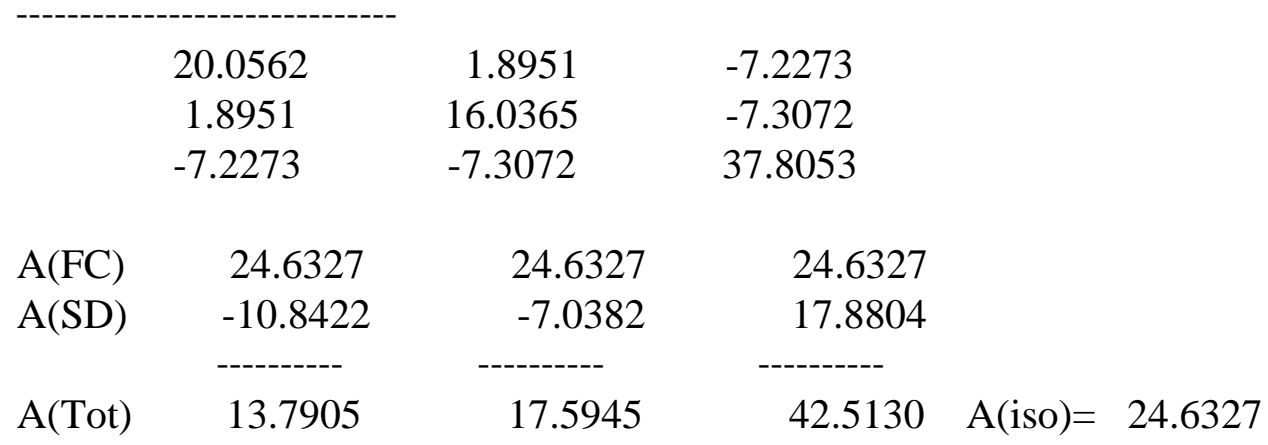

Orientation:

$\begin{array}{llcl}\mathrm{X} & -0.0704620 & 0.9463009 & -0.3155150 \\ \mathrm{Y} & -0.9482113 & -0.1617514 & -0.2733712 \\ \mathrm{Z} & -0.3097264 & 0.2799126 & 0.9086905\end{array}$

Note: Tensor is right-handed

Nucleus V : A:ISTP $=51 \mathrm{I}=3.5 \mathrm{P}=140.5173 \mathrm{MHz} / \mathrm{au}^{* *} 3$

$\mathrm{Q}: \mathrm{ISTP}=51 \mathrm{I}=3.5 \mathrm{Q}=-0.0430$ barn

Raw HFC matrix (all values in $\mathrm{MHz}$ ):

\begin{tabular}{|c|c|c|c|}
\hline & -274.2607 & -10.8432 & -34.9121 \\
\hline & -10.8432 & -117.4585 & 59.9878 \\
\hline & -34.9121 & 59.9878 & -86.6663 \\
\hline $\mathrm{A}(\mathrm{FC})$ & -159.4618 & -159.4618 & -159.4618 \\
\hline $\mathrm{A}(\mathrm{SD})$ & 124.2595 & -3.1740 & -121.0855 \\
\hline $\mathrm{A}(\mathrm{Tot})$ & -35.2023 & -162.6358 & -280.5473 \\
\hline
\end{tabular}

Orientation:

$\begin{array}{llll}\mathrm{X} & -0.1424786 & -0.1052469 & 0.9841864 \\ \mathrm{Y} & 0.5954134 & -0.8034194 & 0.0002807 \\ \mathrm{Z} & 0.7906849 & 0.5860378 & 0.1771355\end{array}$

Note: Tensor is right-handed 


\section{References}

1. Froncisz, W.; Hyde, J. S. Broadening by strains of lines in the $\mathrm{g}-$ parallel region of $\mathrm{Cu} 2+\mathrm{EPR}$ spectra. J. Chem. Phys. 1980, 73, 3123-3131. 